\title{
Synthesis of Structurally Diverse Bis-Peptide Oligomers
}

\author{
Sharad Gupta, Megan Macala, Christian E. Schafmeister* \\ Department of Chemistry, University of Pittsburgh Pittsburgh, PA 15260 (USA) \\ * To whom correspondence should be addressed; email: meister@pitt.edu
}




\section{Supporting information: Table of contents}

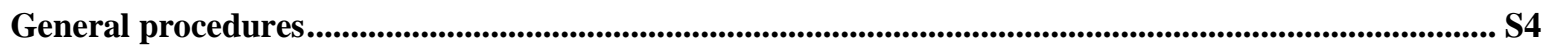

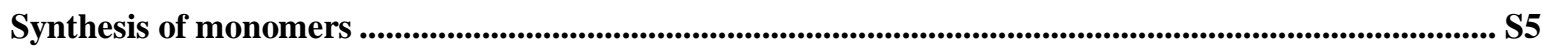

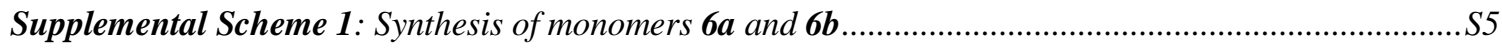

Supplemental Scheme 2: Synthesis of monomers 1 and 2.................................................................. 10

Supplemental Scheme 3: Synthesis of monomer $7 b$...........................................................................

Measurement of kinetics of diketopiperazine formation ...................................................................... S19

Supplemental Scheme 4: Assembly of bis amino acid dimers........................................................... 19

Supplemental Figure 1: Schematic Diagram of the flow cell set up for the kinetic measurements. .........S19

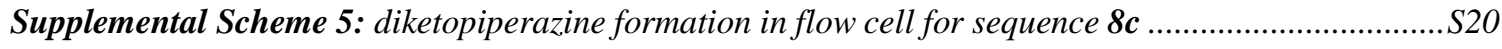

Supplementary Table 1: Data obtained for on resin DKP closure for sequence $6 c$.................................S22

Supplemental Scheme 6: Cleavage of the product $\mathbf{s} 9$ following the DKP formation reaction...................S23

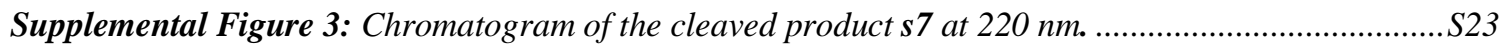

Microwave assisted DKP closure in oligomer 10.................................................................................................. S24

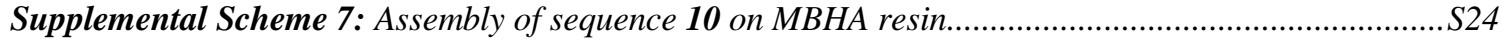

On the resin diketopiperazine formation in oligomer 10 via microwave irradiation. ...............................S25

Supplemental Figure 4: A representative chromatogram for the cleaved product from diketopiperazine closure in oligomer 10 at $274 \mathrm{~nm}$ (60 min microwave irradiation) .......................................................S25

Supplemental Table 2: Areas under the major peaks observed in chromotograms with varying microwave irradiation time $(t)$

Supplementary Figure 5: Plot of $\ln (F)$ against MW irradiation time $(t)$............................................... 227

Supplemental Scheme 8: Assembly of 5-mer homosequence 13 on MBHA resin..................................S28

HPLC chromotograms for the oligomers before purification............................................................... S28

Supplemental Figure 6: HPLC chromatogram for homo-oligomer 14 at $220 \mathrm{~nm}$..................................S28

Supplemental Figure 7: HPLC chromatogram for hetero-oligomer 3 at 220 nm...................................S29

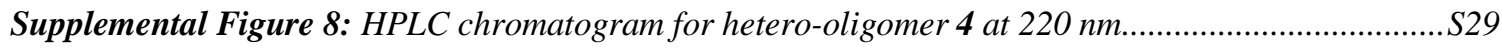

Supplemental Figure 9: HPLC chromatogram for hetero-oligomer 19 at $220 \mathrm{~nm}$.....................................S30

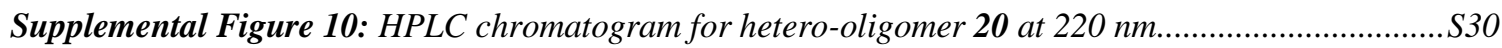

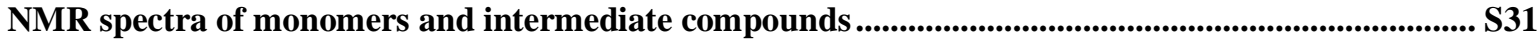

Supplemental Figure 11: $1 \mathrm{H}$ spectrum of compound s2, $300 \mathrm{MHz}$, DMSO-d6, 350K...........................S31

Supplemental Figure 12: Proton decoupled 13C spectrum of compound s2, $75.4 \mathrm{MHz}, \mathrm{DMSO}-\mathrm{d} 6, \mathrm{rt}$...S32

Supplemental Figure 13: DEPT135 spectrum of compound s2, $75.4 \mathrm{MHz}, \mathrm{DMSO}-\mathrm{d6}, \mathrm{rt} \ldots \ldots \ldots \ldots \ldots \ldots \ldots . . . . . . . . . . . .33$

Supplemental Figure 14: $1 \mathrm{H}$ spectrum of compound s4, $300 \mathrm{MHz}$, DMSO-d6, 350K............................S34

Supplemental Figure 15: Proton decoupled 13C spectrum of compound s4, $75.4 \mathrm{MHz}, \mathrm{DMSO}-\mathrm{d} 6, \mathrm{rt} \ldots \mathrm{S} 35$

Supplemental Figure 16: DEPT135 spectrum of compound s4, $75.4 \mathrm{MHz}, \mathrm{DMSO}-\mathrm{d6}, \mathrm{rt} \ldots \ldots \ldots \ldots \ldots \ldots \ldots . . . \mathrm{S} 36$ 
Supplemental Figure 17: $1 \mathrm{H}$ spectrum of compound 6a, $300 \mathrm{MHz}, \mathrm{DMSO}-\mathrm{d6}, 350 \mathrm{~K}$.

Supplemental Figure 18: Proton decoupled 13C spectrum of compound 6a, 75.4 MHz, DMSO-d6, rt...S38

Supplemental Figure 19: DEPT135 spectrum of compound 6a, 75.4 MHz, DMSO-d6, rt .$S 39$

Supplemental Figure 20: $1 \mathrm{H}$ spectrum of compound $\mathbf{6} \boldsymbol{b}, 300 \mathrm{MHz}, \mathrm{DMSO}-\mathrm{d6}, 350 \mathrm{~K}$. S40

Supplemental Figure 21: Proton decoupled 13C spectrum of compound $6 \mathbf{b}, 75.4 \mathrm{MHz}, \mathrm{DMSO}-\mathrm{d} 6, \mathrm{rt} \ldots \mathrm{S} 4 \mathrm{I}$

Supplemental Figure 22: DEPT135 spectrum of compound $6 \boldsymbol{b}, 75.4 \mathrm{MHz}, \mathrm{DMSO}-\mathrm{d6}, \mathrm{rt}$ .$S 42$

Supplemental Figure 23: $1 \mathrm{H}$ spectrum of compound s5, $300 \mathrm{MHz}, \mathrm{DMSO}-\mathrm{d6}, 350 \mathrm{~K}$. .S43

Supplemental Figure 24: Proton decoupled 13C spectrum of compound s5, 75.4 MHz, DMSO-d6, rt ...S44

Supplemental Figure 25: DEPT135 spectrum of compound s5, $75.4 \mathrm{MHz}, \mathrm{DMSO}-\mathrm{d6}, \mathrm{rt}$ .$S 45$

Supplemental Figure 26: $1 \mathrm{H}$ spectrum of compound s6, $300 \mathrm{MHz}, \mathrm{DMSO}-\mathrm{d6}, 350 \mathrm{~K}$. .$S 46$

Supplemental Figure 27: Proton decoupled 13C spectrum of compound s6, 75.4 MHz, DMSO-d6, rt ...S47 Supplemental Figure 28: DEPT135 spectrum of compound s6, 75.4 MHz, DMSO-d6, rt .$S 48$

Supplemental Figure 29: $1 \mathrm{H}$ spectrum of compound $\mathbf{s}$, $300 \mathrm{MHz}, \mathrm{DMSO}-\mathrm{d6}, 350 \mathrm{~K}$. .S49

Supplemental Figure 30: Proton decoupled 13C spectrum of compound s7, 75.4 MHz, DMSO-d6, rt ...S50

Supplemental Figure 31: DEPT135 spectrum of compound s7, 75.4 MHz, DMSO-d6, rt .$S 51$

Supplemental Figure 32: $1 \mathrm{H}$ spectrum of compound s8, $300 \mathrm{MHz}, \mathrm{DMSO}-\mathrm{d6}, 350 \mathrm{~K}$. .$S 52$

Supplemental Figure 33: Proton decoupled 13C spectrum of compound s8, 75.4 MHz, DMSO-d6, rt ...S53

Supplemental Figure 34: DEPT135 spectrum of compound s8, 75.4 MHz, DMSO-d6, rt .S54

Supplemental Figure 35: $1 \mathrm{H}$ spectrum of compound 1, $300 \mathrm{MHz}, \mathrm{DMSO}-\mathrm{d6}, 350 \mathrm{~K}$. S55

Supplemental Figure 36: Proton decoupled 13C spectrum of compound 1, 75.4 MHz, DMSO-d6, rt....S56

Supplemental Figure 37: DEPT135 spectrum of compound 1, 75.4 MHz, DMSO-d6, rt .... .$S 57$

Supplemental Figure 38: $1 \mathrm{H}$ spectrum of compound 2, $300 \mathrm{MHz}, \mathrm{DMSO}-\mathrm{d6}, 350 \mathrm{~K}$ .558

Supplemental Figure 39: Proton decoupled 13C spectrum of compound 2, 75.4 MHz, DMSO-d6, rt.....S59

Supplemental Figure 40: DEPT135 spectrum of compound 2, 75.4 MHz, DMSO-d6, rt .560

Supplemental Figure 41: $1 \mathrm{H}$ spectrum of compound 7b, $300 \mathrm{MHz}, \mathrm{DMSO}-\mathrm{d6}, 350 \mathrm{~K}$. .S61

Supplemental Figure 42: Proton decoupled 13C spectrum of compound 7b, 75.4 MHz, DMSO-d6, rt ...S62 Supplemental Figure 43: DEPT135 spectrum of compound 7b, 75.4 MHz, DMSO-d6, rt .563 


\section{General procedures}

THF was distilled from $\mathrm{Na}$ /benzophenone under nitrogen atmosphere. $\mathrm{CH}_{2} \mathrm{Cl}_{2}$ was distilled from $\mathrm{CaH}_{2}$. All other reagents were used as received unless otherwise noted. All the reactions were carried out in flame-dried or oven-dried glassware under nitrogen atmosphere unless otherwise noted. Column chromatography was performed either manually using 32-63 D (60 A particle size) grade silica gel or using commercially available normal phase flash chromatography silica gel columns on automated purification system. Analytical TLC analysis was performed on Silica Gel plates $(250 \mu \mathrm{m}$ thickness). NMR experiments were performed on $300 \mathrm{MHz}$ spectrometer. Chemical shifts $(\delta)$ are reported in parts per million (ppm) relative to DMSO- $d_{6}$ residual solvent peaks. If possible, rotational isomers were resolved by obtaining spectra at $77{ }^{\circ} \mathrm{C}(350 \mathrm{~K})$ in DMSO- $d_{6}$. IR spectra were obtained on a FT-IR instrument. Optical rotations were obtained at ambient temperature $\left(23 \pm 2{ }^{\circ} \mathrm{C}\right)$. HPLC-MS analysis was performed on a $\mathrm{C}_{18}$ column (3.5 $\mu \mathrm{m}$ packing, $4.6 \mathrm{~mm}$ x $150 \mathrm{~mm}$ ) coupled to a Mass Spectrometer (ESI source). The elemental composition of purified compounds was confirmed by the spectrums obtained on a high resolution mass spectrometer with an electrospray ion source (HRESIQTOFMS). Purification by preparative HPLC was carried out on a semiprep $\mathrm{C}_{18}$ column (5 $\mu \mathrm{m}$ packing, $10 \mathrm{~mm} \times 100 \mathrm{~mm}$ ).

Solid phase chemistry was performed using a home-built manual synthesizer. Dry dichloromethane used in coupling reactions was obtained from distillation over $\mathrm{CaH} 2$. Diisopropylethyl amine (DIPEA) was distilled under nitrogen sequentially from ninhydrin and potassium hydroxide and stored over molecular sieves (4 A). All solid phase reactions were mixed by bubbling argon through the reaction mixture. 


\section{Synthesis of monomers}

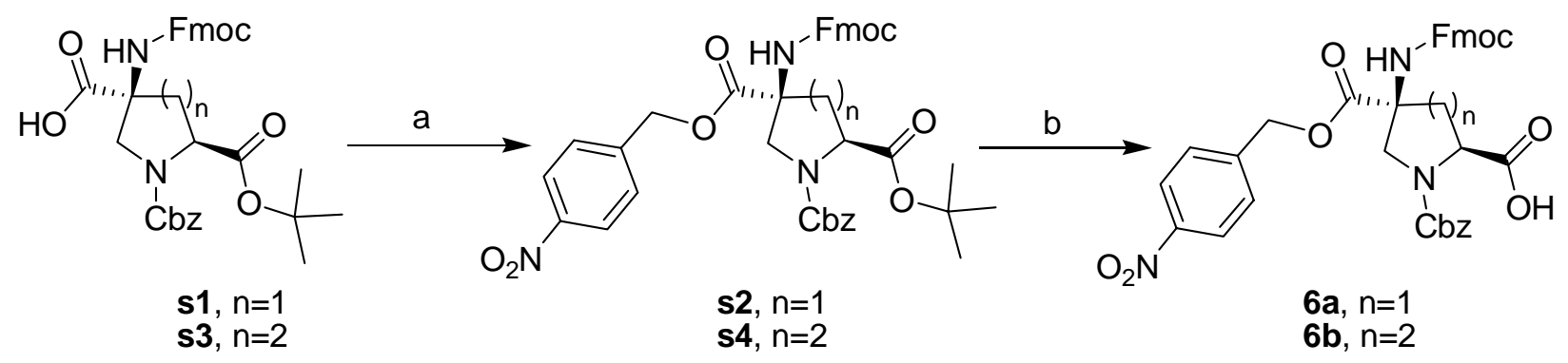

Reagents and Reaction conditions:

$\square$ (a) $\mathrm{NaHCO}_{3}, \mathrm{KI}, p$-nitrobenzyl bromide, DMF, rt ,12h; (b) $3: 7 \mathrm{TFA} / \mathrm{CH}_{2} \mathrm{Cl}_{2}$, rt, $2 \mathrm{~h}$.

Supplemental Scheme 1: Synthesis of monomers $6 \mathbf{a}$ and $\mathbf{6 b}$

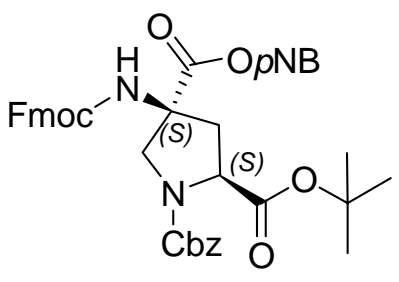

s2

(2S,4S)-4-(9H-Fluoren-9-ylmethoxycarbonylamino)-proline-1,2,4-tricarboxylic acid

\section{1-benzyl ester 4- ( $p$-nitro)benzyl ester 2-tert-butyl ester (s2)}

To a suspension of $\mathbf{s 1}^{\mathbf{1}}$ (418 $\left.\mathrm{mg}, 0.71 \mathrm{mmol}\right), \mathrm{KI}(119 \mathrm{mg}, 0.71 \mathrm{mmol}, 1$ equiv.) and $\mathrm{NaHCO}_{3}(57 \mathrm{mg}, 0.67 \mathrm{mmol}, 0.9$ equiv.) in anhydrous DMF $(5 \mathrm{~mL}, 7 \mathrm{~mL}$ per mmol of substrate) was added $p$-nitrobenzyl bromide ( $383 \mathrm{mg}, 1.8 \mathrm{mmol}, 2.5$ equiv.) in one portion. The stirring was continued at room temperature and the reaction progress was monitored by HPLC-MS. After completion of reaction (about 10 hours), $15 \mathrm{~mL}$ of water was added to the reaction mixture and extracted with EtOAc $(30 \mathrm{~mL}+2 \times 10 \mathrm{~mL})$. The organic layers were combined and washed with brine $(10 \mathrm{~mL})$. This solution was dried over anhydrous $\mathrm{Na}_{2} \mathrm{SO}_{4}$, filtered, and concentrated by rotary evaporation. The crude product was purified by FC (10g silica gel column, 5-35\% EtOAc in hexane gradient

(1) Levins, C. G.; Schafmeister, C. E. J. Am. Chem. Soc. 2003, 125, 4702-4703. 
over $60 \mathrm{CVs}$ ). The fractions containing pure compound were combined, concentrated and the residual solvent was removed by applying high vacuum overnight to yield s2 (411 mg, $5.7 \mathrm{mmol}, 80 \%)$ as white foamy solid: $[\alpha]^{23}{ }_{\mathrm{D}}=+11.0^{\circ}\left(c 1.15, \mathrm{CHCl}_{3}\right)$; IR (film) $v_{\max }$ $3317,2977,1711,1523,1449,1417,1347,1259,1156,749,740,697 \mathrm{~cm}^{-1} ;{ }^{1} \mathrm{H}$ NMR $\left(300 \mathrm{MHz}, 77^{\circ} \mathrm{C}, \mathrm{DMSO}-d_{6}\right) \delta 8.07(\mathrm{~d}, J=8.4 \mathrm{~Hz}, 2 \mathrm{H}), 8.00(\mathrm{~s}, 1 \mathrm{H}), 7.85(\mathrm{~d}, J=7.5 \mathrm{~Hz}$, 2H), $7.64(\mathrm{~d}, J=7.5 \mathrm{~Hz}, 2 \mathrm{H}), 7.53(\mathrm{~d}, J=8.4 \mathrm{~Hz}, 2 \mathrm{H}), 7.26-7.42(\mathrm{~m}, 9 \mathrm{H}), 5.22(\mathrm{br} \mathrm{s}$, 2H), 5.08 (br s, 2H), 4.03-4.39 (m, 5H), $3.64(\mathrm{~d}, J=11.4 \mathrm{~Hz}, 1 \mathrm{H}), 2.87$ (br m, 1H), 2.33 (br m, $1 \mathrm{H}), 1.37(\mathrm{~s}, 9 \mathrm{H}) ;{ }^{13} \mathrm{C} \mathrm{NMR}\left(75.4 \mathrm{MHz}, 20{ }^{\circ} \mathrm{C}\right.$, DMSO- $\left.d_{6}\right)$ : mixture of rotamers $\delta$ 171.0, 170.1, 169.8, 155.8, 153.5, 153.2, 146.9, 143.4, 143.1, 140.6, 136.5 and 136.3, $128.4,128.1,127.8,127.7,127.5,127.4,127.2,126.9,125.0,123.2,120.0,81.0,66.2$ $\left(\mathrm{CH}_{2}\right), 65.8\left(\mathrm{CH}_{2}\right), 65.5\left(\mathrm{CH}_{2}\right), 63.1$ and 62.2, 58.3 and $58.0(\mathrm{CH}), 54.7$ and $54.3\left(\mathrm{CH}_{2}\right)$, $46.4(\mathrm{CH}), 38.6$ and $37.6\left(\mathrm{CH}_{2}\right), 27.4$ and $27.3\left(\mathrm{CH}_{3}, 3 \mathrm{C}\right)$; HPLC-MS: MeCN $(0.05 \%$ Formic acid) / water ( $0.1 \%$ Formic Acid), $5 \%$ to $95 \% \mathrm{MeCN}$ over $30 \mathrm{~min}$; flow rate 0.80 $\mathrm{mL} / \mathrm{min}$; UV detection at $274 \mathrm{~nm}$; $\mathrm{t}_{\mathrm{R}}$ for $\mathbf{s 2} 29.09 \mathrm{~min}$; HRESIQTOFMS calcd for $\mathrm{C}_{40} \mathrm{H}_{39} \mathrm{~N}_{3} \mathrm{O}_{10} \mathrm{Na}\left(\mathrm{M}+\mathrm{Na}^{+}\right)$744.2533, found 744.2532.

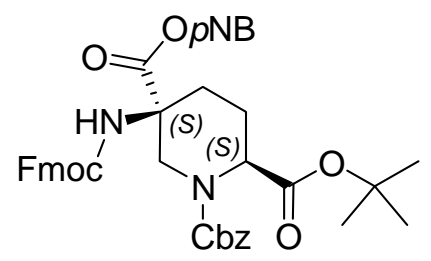

s4

(2S,5S)-5-(9H-Fluoren-9-ylmethoxycarbonylamino)-piperidine-1,2,5-tricarboxylic acid 1-benzyl ester 5-(p-nitro)benzyl ester 2-tert-butyl ester (s4) 
$129 \mathrm{mg}(0.21 \mathrm{mmol})$ of $\mathbf{s 3}^{2}$ yielded $112 \mathrm{mg}(0.16 \mathrm{mmol}, 76 \%)$ of $\mathbf{s} 4$ as white foamy solid according to the procedure given above for $\mathbf{s 2}:[\alpha]^{23} \mathrm{D}=-9.1^{\circ}\left(c 1.5, \mathrm{CHCl}_{3}\right) ; \mathrm{IR}$ (film) $v_{\max } 3333,3030,2955,1716,1521,1449,1347,1317,1254,749,741,667 \mathrm{~cm}^{-1} ;{ }^{1} \mathrm{H}$ NMR $\left(300 \mathrm{MHz}, 77{ }^{\circ} \mathrm{C}, \mathrm{DMSO}-d_{6}\right) \delta 8.06(\mathrm{~d}, J=8.7 \mathrm{~Hz}, 2 \mathrm{H}), 7.85(\mathrm{~d}, J=7.5 \mathrm{~Hz}, 2 \mathrm{H}), 7.80(\mathrm{~s}$, 1H), $7.65(\mathrm{~d}, J=7.5 \mathrm{~Hz}, 2 \mathrm{H}), 7.52(\mathrm{~d}, J=8.4 \mathrm{~Hz}, 2 \mathrm{H}), 7.39(\mathrm{dd}, J=7.5,7.5 \mathrm{~Hz}, 2 \mathrm{H})$, 7.26-7.31 (m, 7H), 5.17 (br s, 2H), 5.08 (br s, 2H), 4.71 (br d, J = 13.5 Hz, 1H), 4.64 (m, 1H), 4.30-4.33 (m, 2H), $4.16(\mathrm{t}, J=6.6 \mathrm{~Hz}, 1 \mathrm{H}) 2.99$ (br d, $J=13.2 \mathrm{~Hz}, 1 \mathrm{H}), 2.24$ (br d, $J$ $=13.5 \mathrm{~Hz}, 1 \mathrm{H}), 2.04(\mathrm{br} \mathrm{d}, J=14.1 \mathrm{~Hz}, 1 \mathrm{H}), 1.86($ br m, $1 \mathrm{H}), 1.63(\mathrm{br} \mathrm{dd}, J=12.9,12.9$, $\mathrm{Hz}, 1 \mathrm{H}), 1.42(\mathrm{~s}, 9 \mathrm{H}) ;{ }^{13} \mathrm{C}$ NMR $\left(75.4 \mathrm{MHz}, 20{ }^{\circ} \mathrm{C}\right.$, DMSO- $\left.d_{6}\right)$ : mixture of rotamers $\delta$ $170.9,169.8,169.5,155.1,155.0,154.7,146.9,143.6,143.5,140.7,136.6,128.3,128.2$ 127.7, 127.6, 127.2, 126.9, 125.0, 123.3, 120.1, 81.4, $66.4\left(\mathrm{CH}_{2}\right), 65.6\left(\mathrm{CH}_{2}\right), 64.8\left(\mathrm{CH}_{2}\right)$, 56.3, 56.2, 53.7 and 53.5(CH), $46.6\left(\mathrm{CH}_{2}\right), 46.5(\mathrm{CH}), 29.0$ and $28.8\left(\mathrm{CH}_{2}\right), 27.5$ and 27.4 $\left(\mathrm{CH}_{3}, 3 \mathrm{C}\right), 22.4$ and $22.3\left(\mathrm{CH}_{2}\right)$; HPLC-MS: $\mathrm{MeCN}(0.05 \%$ Formic acid) / water $(0.1 \%$ Formic Acid), $5 \%$ to $95 \%$ MeCN over $30 \mathrm{~min}$; flow rate $0.80 \mathrm{~mL} / \mathrm{min}$; UV detection at $274 \mathrm{~nm}$; $\mathrm{t}_{\mathrm{R}}$ for $\mathbf{s 4}, 29.50 \mathrm{~min}$; HRESIQTOFMS calcd for $\mathrm{C}_{41} \mathrm{H}_{41} \mathrm{~N}_{3} \mathrm{O}_{10} \mathrm{Na}\left(\mathrm{M}+\mathrm{Na}^{+}\right)$ 758.2690, found 758.2698.

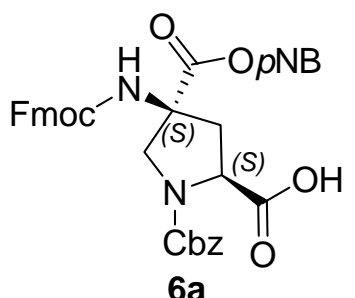

(2S,4S)-4-(9H-Fluoren-9-ylmethoxycarbonylamino)-proline-1,2,4-tricarboxylic acid 1-benzyl ester 4- (p-nitro)benzyl ester (6a)

$\left(^{2}\right)$ Gupta, S.; Das, B. C.; Schafmeister, C. E. Org. Lett. 2004, 6, 3369-3371. 
$205 \mathrm{mg}(0.28 \mathrm{mmol})$ of $\mathbf{s} 2$ was dissolved in $6 \mathrm{~mL}$ of $3: 7$ mixture of TFA/ DCM (20 mL per mmol of substrate) and stirring continued for 2 hours at room temperature. After confirming the disappearance of substrate by $\mathrm{TLC}\left(5: 1 \mathrm{CHCl}_{3} / \mathrm{MeOH}\right.$, visualized under $\mathrm{UV}$ ), reaction mixture was diluted with $6 \mathrm{~mL}$ of toluene and evaporated by the use of rotary evaporator. This step of adding toluene and evaporation in vacuo was repeated one additional time to ensure complete removal of TFA. The crude product was purified by FC (10g silica gel column, $0-10 \% \mathrm{CHCl}_{3}$ in $\mathrm{MeOH}$ gradient over $\left.60 \mathrm{CVs}\right)$. The fractions containing pure compound were combined, concentrated and the residual solvent was removed by applying high vacuum to yield $\mathbf{6 a}(175 \mathrm{mg}, 0.26 \mathrm{mmol}, 93 \%)$ as a white foamy solid: $[\alpha]^{23}{ }_{D}=-18.0^{\circ}\left(c 2.4, \mathrm{CHCl}_{3}\right)$; IR (film) $v_{\max } 3307,3033,2953,1709,1523$, $1450,1422,1348,1263,749,741,697 \mathrm{~cm}^{-1} ;{ }^{1} \mathrm{H}$ NMR $\left(300 \mathrm{MHz}, 77^{\circ} \mathrm{C}\right.$, DMSO- $\left.d_{6}\right) \delta$ 8.05 (d, $J=8.4 \mathrm{~Hz}, 3 \mathrm{H}$, overlap with Fmoc-NH-), 7.84 (d, $J=7.5 \mathrm{~Hz}, 2 \mathrm{H}), 7.65$ (d, $J=$ $7.5 \mathrm{~Hz}, 2 \mathrm{H}), 7.52(\mathrm{~d}, J=8.4 \mathrm{~Hz}, 2 \mathrm{H}), 7.27-7.42(\mathrm{~m}, 9 \mathrm{H}), 5.22(\mathrm{br} \mathrm{s}, 2 \mathrm{H}), 5.08$ (br s, 2H), 4.13-4.37 (m, 5H), 3.61 (d, $J=11.4 \mathrm{~Hz}, 1 \mathrm{H}), 2.90$ (br m, 1H), 2.33 (br m, 1H); ${ }^{13} \mathrm{C} \mathrm{NMR}$ (75.4 MHz, $20{ }^{\circ} \mathrm{C}$, DMSO- $\left.d_{6}\right)$ : mixture of rotamers $\delta 172.7,172.4,171.3,156.0,153.7$, 153.5, 147.0, 143.6, 143.2, 140.7, 136.6, 128.5, 128.3, 127.9, 127.6, 127.5, 127.0, 125.2, 123.3, 120.1, 79.2, $66.3\left(\mathrm{CH}_{2}\right), 66.0\left(\mathrm{CH}_{2}\right), 65.5\left(\mathrm{CH}_{2}\right), 63.0$ and 62.2, 57.8 and 57.5 $(\mathrm{CH}), 54.9$ and $54.5\left(\mathrm{CH}_{2}\right), 46.5(\mathrm{CH}), 38.7$ and $37.6\left(\mathrm{CH}_{2}\right)$; HPLC-MS: $\mathrm{MeCN}(0.05 \%$ Formic acid) / water ( $0.1 \%$ Formic Acid), $5 \%$ to $95 \% \mathrm{MeCN}$ over $30 \mathrm{~min}$; flow rate 0.80 $\mathrm{mL} / \mathrm{min}$; UV detection at $274 \mathrm{~nm}$; $\mathrm{t}_{\mathrm{R}}$ for $\mathbf{6 a}, 25.21 \mathrm{~min}$; HRESIQTOFMS calcd for $\mathrm{C}_{36} \mathrm{H}_{31} \mathrm{~N}_{3} \mathrm{O}_{10} \mathrm{Na}\left(\mathrm{M}+\mathrm{Na}^{+}\right)$688.1907, found 688.1906. 


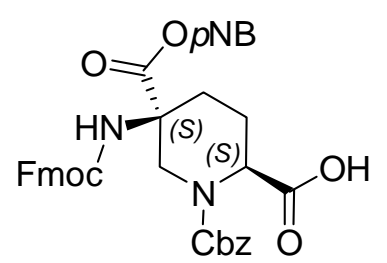

$6 b$

(2S,5S)-5-(9H-Fluoren-9-ylmethoxycarbonylamino)-piperidine-1,2,5-tricarboxylic acid 1-benzyl ester 5- ( $p$-nitro)benzyl ester (6b)

$112 \mathrm{mg}(0.15 \mathrm{mmol})$ of $\mathbf{s} 4$ yielded $95 \mathrm{mg}(14.0 \mathrm{mmol}, 92 \%)$ of $\mathbf{6 b}$ as a white foamy solid according to the procedure given above for $\mathbf{6 a}:[\alpha]^{23}{ }_{\mathrm{D}}=-6.4^{\circ}\left(c 0.8, \mathrm{CHCl}_{3}\right)$; IR (film) $v_{\max } 3333,3030,2955,1716,1521,1449,1347,1317,1254,749,741,667 \mathrm{~cm}^{-1} ;{ }^{1} \mathrm{H}$ NMR $\left(300 \mathrm{MHz}, 77^{\circ} \mathrm{C}, \mathrm{DMSO}-d_{6}\right) \delta 8.05(\mathrm{~d}, J=8.4 \mathrm{~Hz}, 2 \mathrm{H}), 7.85(\mathrm{~d}, J=7.2 \mathrm{~Hz}, 2 \mathrm{H}), 7.78(\mathrm{~s}$, 1H), $7.65(\mathrm{~d}, J=7.2 \mathrm{~Hz}, 2 \mathrm{H}), 7.52(\mathrm{~d}, J=8.1 \mathrm{~Hz}, 2 \mathrm{H}), 7.27-7.42(\mathrm{~m}, 9 \mathrm{H}), 5.15(\mathrm{br} \mathrm{s}$, 2H), 5.07 (br s, 2H), 4.70-4.74 (m, 2H), $4.29(\mathrm{~d}, J=6.6 \mathrm{~Hz}, 2 \mathrm{H}), 4.16(\mathrm{t}, J=6.6 \mathrm{~Hz}, 1 \mathrm{H})$ $3.05(\operatorname{brd}, J=13.2 \mathrm{~Hz}, 1 \mathrm{H}), 2.25($ br d, $J=13.2 \mathrm{~Hz}, 1 \mathrm{H}), 2.08($ br d, $J=11.4 \mathrm{~Hz}, 1 \mathrm{H})$, $1.88(\mathrm{br} \mathrm{m}, 1 \mathrm{H}) 1.63(\mathrm{br} \mathrm{dd}, J=12.9,12.9, \mathrm{~Hz}, 1 \mathrm{H}),{ }^{13} \mathrm{C} \mathrm{NMR}\left(75.4 \mathrm{MHz}, 20{ }^{\circ} \mathrm{C}\right.$, DMSO- $\left.d_{6}\right)$ : mixture of rotamers $\delta 172.3,172.2,171.0,155.2,155.0,154.7,146.9,143.7$, 143.6, 140.7, 136.7, 128.4, 128.3, 127.7, 127.6, 127.2, 127.1, 127.0, 125.1, 123.3, 120.1, 79.2, $66.4\left(\mathrm{CH}_{2}\right), 65.7\left(\mathrm{CH}_{2}\right), 64.8\left(\mathrm{CH}_{2}\right), 56.4$ and 56.3, $53.0(\mathrm{CH}), 46.5\left(\mathrm{CH}_{2}\right), 46.4$ (CH), $29.3\left(\mathrm{CH}_{2}\right), 22.5\left(\mathrm{CH}_{2}\right)$; HPLC-MS: $\mathrm{MeCN}(0.05 \%$ Formic acid) / water $(0.1 \%$ Formic Acid), $5 \%$ to $95 \% \mathrm{MeCN}$ over $30 \mathrm{~min}$; flow rate $0.80 \mathrm{~mL} / \mathrm{min}$; UV detection at $274 \mathrm{~nm}$; $\mathrm{t}_{\mathrm{R}}$ for $\mathbf{6 b}, 25.75 \mathrm{~min}$; HRESIQTOFMS calcd for $\mathrm{C}_{37} \mathrm{H}_{33} \mathrm{~N}_{3} \mathrm{O}_{10} \mathrm{Na}\left(\mathrm{M}+\mathrm{Na}^{+}\right)$ 702.2064, found 702.2042. 


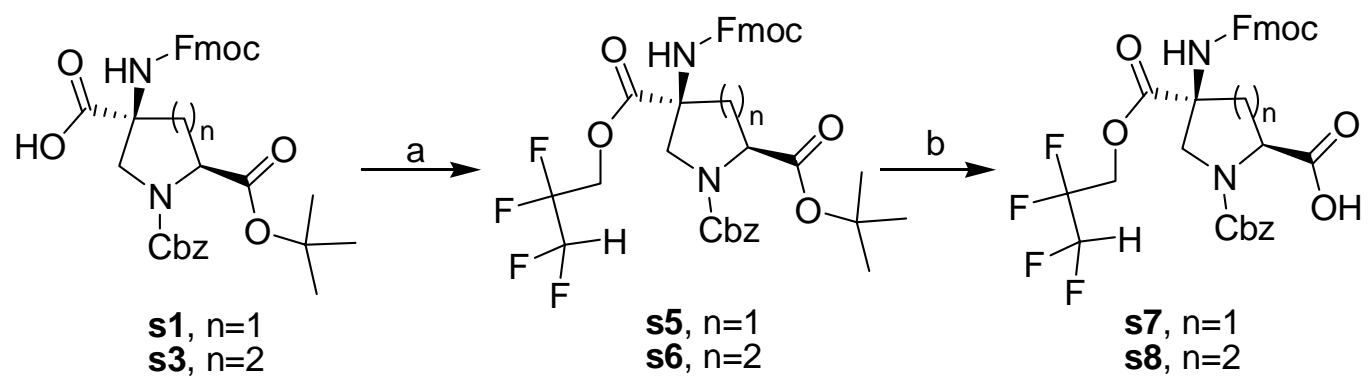

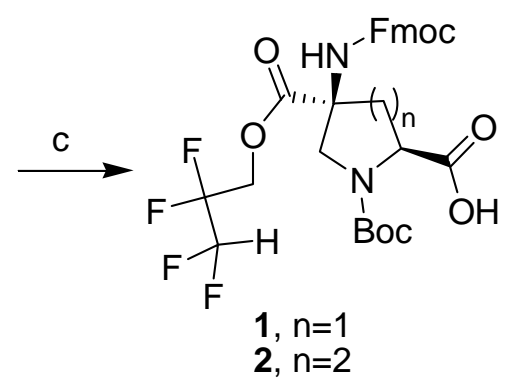

Reagents and Reaction conditions:

$\square$ (a) DCC, DMAP, $\mathrm{CF}_{2} \mathrm{HCF}_{2} \mathrm{CH}_{2} \mathrm{OH}, \mathrm{CH}_{2} \mathrm{Cl}_{2}, 0^{\circ} \mathrm{C}$ to rt, $12 \mathrm{~h}$; (b) $3: 7 \mathrm{TFA} / \mathrm{CH}_{2} \mathrm{Cl}_{2}, \mathrm{rt}, 2 \mathrm{~h}$;

(c) $\mathrm{H}_{2}, 10$ wt. \% Pd/C, $\mathrm{Boc}_{2} \mathrm{O}$, DIPEA, THF, rt.

Supplemental Scheme 2: Synthesis of monomers 1 and 2

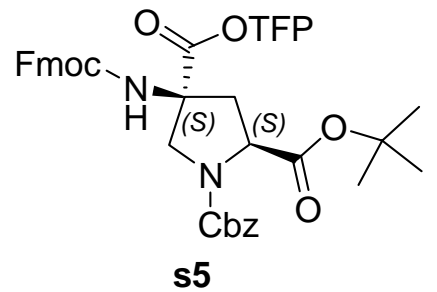

(2S,4S)-5-(9H-Fluoren-9-ylmethoxycarbonylamino)-proline-1,2,4-tricarboxylic acid

\section{1-benzyl ester 4-(2,2,3,3-tetrafluoro) propyl ester 2-tert-butyl ester (s5)}

To a solution of $\mathbf{s} 1(9.57 \mathrm{~g}, 16.3 \mathrm{mmol})$ in dry DCM $(163 \mathrm{~mL}, 10 \mathrm{~mL}$ per mmol) was added DMAP (100 mg, $0.82 \mathrm{mmol}, 0.05$ equiv.) and 2,2,3,3-tetraflouro propanol ( 2,93 mL, 32.6 mmol, 2 equiv.). This solution was cooled in ice bath under $\mathrm{N}_{2}$ and DCC (4.04 $\mathrm{g}, 19.6 \mathrm{mmol}, 1.2$ equiv.) was added in one portion. The reaction mixture was allowed to warm up to room temperature and stirring continued overnight. Disappearance of substrate was confirmed by TLC analysis $\left(5: 1 \mathrm{CHCl}_{3}: \mathrm{MeOH}\right.$, observed under UV). The 
reaction was quenched by the addition of $20 \mathrm{~mL}$ of $10 \%$ acetic acid in DCM and solvent was evaporated by use of rotary evaporator. The residue was dissolved in $100 \mathrm{~mL}$ of 1:1 EtOAc / hexane mixture and filtered through sintered funnel to remove byproduct DCU. The filtrate was concentrated and purified by FC (two 40g silica gel columns, 5-35\% EtOAc in hexane gradient over $40 \mathrm{CVs}$ ). The fractions containing pure compound were combined, concentrated and the residual solvent was removed by applying high vacuum to yield s5 $(9.7 \mathrm{~g}, 13.84 \mathrm{mmol}, 85 \%)$ as white foamy solid; $[\alpha]^{23}{ }_{\mathrm{D}}=+2.9^{\circ}(c 1.09$, $\mathrm{CHCl}_{3}$ ); IR (film) $v_{\max } 3307,2979,1712,1525,1450,1419,1258,1157,1109,759,741$, $698 \mathrm{~cm}^{-1} ;{ }^{1} \mathrm{H}$ NMR $\left(300 \mathrm{MHz}, 77{ }^{\circ} \mathrm{C}\right.$, DMSO- $\left.d_{6}\right) \delta 7.99(\mathrm{~s}, 1 \mathrm{H}), 7.85(\mathrm{~d}, J=7.8 \mathrm{~Hz}, 2 \mathrm{H})$, $7.66(\mathrm{~d}, J=7.5 \mathrm{~Hz}, 2 \mathrm{H}), 7.31-7.40(\mathrm{~m}, 9 \mathrm{H}), 6.40\left(\mathrm{tt}, J=52.2 \mathrm{~Hz}, J=5.1 \mathrm{~Hz},-\mathrm{CF}_{2} \mathrm{CF}_{2} H\right.$, 1H) $5.08($ br s, $2 \mathrm{H}), 4.56\left(\mathrm{t}, J=13.8 \mathrm{~Hz},-\mathrm{CH}_{2} \mathrm{CF}_{2^{-}}, 2 \mathrm{H}\right), 4.28-4.42(\mathrm{~m}, 3 \mathrm{H}), 4.19(\mathrm{t}, J=$ $6.4 \mathrm{~Hz}, 1 \mathrm{H}), 4.03(\mathrm{~d}, J=11.4 \mathrm{~Hz}, 1 \mathrm{H}), 3.61(\mathrm{~d}, J=11.4 \mathrm{~Hz}, 1 \mathrm{H}), 2.82(\mathrm{br}, 1 \mathrm{H}), 2.32(\mathrm{br}$, 1H), 1.35 (br s, $9 \mathrm{H}) ;{ }^{13} \mathrm{C}$ NMR $\left(75.4 \mathrm{MHz}, 20^{\circ} \mathrm{C}\right.$, DMSO- $\left.d_{6}\right)$ : mixture of rotamers $\delta$ $170.1,169.7,159.2,143.5,140.6,136.5,136.3,128.2,128.1,127.6,127.5,127.2,126.8$, 124.9, 119.9, $109.0\left(\mathrm{tt},{ }^{1} J=248 \mathrm{~Hz},{ }^{2} J=34 \mathrm{~Hz}, \mathrm{CH}\right), 80.9,66.2\left(\mathrm{CH}_{2}\right), 65.7\left(\mathrm{CH}_{2}\right), 63.0$ and 62.1, $60.2\left(\mathrm{t},{ }^{2} J=28 \mathrm{~Hz}, \mathrm{CH}_{2}\right), 58.3$ and $57.9(\mathrm{CH}), 54.5$ and $54.1\left(\mathrm{CH}_{2}\right), 46.4(\mathrm{CH})$, 38.7 and $37.3\left(\mathrm{CH}_{2}\right), 27.4\left(\mathrm{CH}_{3}, 3 \mathrm{C}\right)$; HPLC-MS: $\mathrm{MeCN}(0.05 \%$ Formic acid $) /$ water (0.1\% Formic Acid), $5 \%$ to $95 \% \mathrm{MeCN}$ over $30 \mathrm{~min}$; flow rate $0.80 \mathrm{~mL} / \mathrm{min}$; UV detection at $274 \mathrm{~nm}$; $\mathrm{t}_{\mathrm{R}}$ for $\mathbf{s 5}, 29.13 \mathrm{~min}$; HRESIQTOFMS calcd for $\mathrm{C}_{36} \mathrm{H}_{36} \mathrm{~N}_{2} \mathrm{O}_{8} \mathrm{~F}_{4} \mathrm{Na}$ $\left(\mathrm{M}+\mathrm{Na}^{+}\right) 723.2305$, found 723.2295 . 


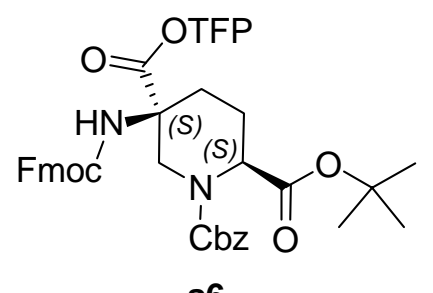

s6

(2S,5S)-5-(9H-Fluoren-9-ylmethoxycarbonylamino)-piperidine-1,2,5-tricarboxylic acid 1-benzyl ester 5- (2,2,3,3-tetrafluoro) propyl ester 2-tert-butyl ester (s6) $4.0 \mathrm{~g}(6.66 \mathrm{mmol})$ of $\mathbf{s} 3$ yielded $3.97 \mathrm{~g}(5.55 \mathrm{mmol}, 83 \%)$ of $\mathbf{s 6}$ as a white foamy solid according to the procedure given above for s5: $[\alpha]^{23}{ }_{\mathrm{D}}=-14.7^{\circ}\left(c 0.9, \mathrm{CHCl}_{3}\right)$; IR (film) $v_{\max } 3316,2977,1761,1729,1526,1450,1321,1255,1118,759,741,698 \mathrm{~cm}^{-1} ;{ }^{1} \mathrm{H}$ NMR $\left(300 \mathrm{MHz}, 77^{\circ} \mathrm{C}, \mathrm{DMSO}-d_{6}\right) \delta 7.84$ (d, $J=7.8 \mathrm{~Hz}, 3 \mathrm{H}$,overlap with Fmoc-NH-), 7.67 (d, $J=7.5 \mathrm{~Hz}, 2 \mathrm{H}), 7.30-7.43(\mathrm{~m}, 9 \mathrm{H}), 6.38\left(\mathrm{tt}, J=52.2,5.1 \mathrm{~Hz},-\mathrm{CF}_{2} \mathrm{CF}_{2} \mathrm{H}, 1 \mathrm{H}\right) 5.12$ (br s, 2H), 4.19-4.70 (m, 4H), $4.35(\mathrm{~d}, J=6.3 \mathrm{~Hz}, 2 \mathrm{H}), 4.21(\mathrm{t}, J=6.3 \mathrm{~Hz}, 1 \mathrm{H}), 2.95(\mathrm{br} \mathrm{d}, J=$ $12.9 \mathrm{~Hz}, 1 \mathrm{H}), 2.17$ (br d, $J=13.2 \mathrm{~Hz}, 1 \mathrm{H}), 2.05(\mathrm{br} \mathrm{d}, J=12.0 \mathrm{~Hz}, 1 \mathrm{H}), 1.82(\mathrm{~m}, 1 \mathrm{H})$ 1.62 (br dd, $J=12.9,12.9, \mathrm{~Hz}, 1 \mathrm{H}), 1.42$ (br s, $9 \mathrm{H}) ;{ }^{13} \mathrm{C}$ NMR $\left(75.4 \mathrm{MHz}, 20{ }^{\circ} \mathrm{C}, \mathrm{DMSO}-\right.$ $\left.d_{6}\right)$ : mixture of rotamers $\delta 170.0,169.6,169.4,155.1,155.0,154.7,143.6,143.5,143.4$, $140.7,136.6,136.5,128.2,127.7,127.5,127.2,126.9,124.9,120.0,81.4,79.1,66.3$ $\left(\mathrm{CH}_{2}\right), 65.5\left(\mathrm{CH}_{2}\right), 59.7\left(\mathrm{CH}_{2}\right), 56.3$ and 56.2, 53.6 and $53.4(\mathrm{CH}), 46.6(\mathrm{CH}), 46.5\left(\mathrm{CH}_{2}\right)$, $28.8\left(\mathrm{CH}_{2}\right), 27.5\left(\mathrm{CH}_{3}, 3 \mathrm{C}\right), 22.2\left(\mathrm{CH}_{2}\right)$; HPLC-MS: MeCN (0.05\% Formic acid) / water (0.1\% Formic Acid), $5 \%$ to $95 \% \mathrm{MeCN}$ over $30 \mathrm{~min}$; flow rate $0.80 \mathrm{~mL} / \mathrm{min}$; UV detection at $274 \mathrm{~nm}$; $\mathrm{t}_{\mathrm{R}}$ for $\mathbf{s 6}, 29.23 \mathrm{~min}$; HRESIQTOFMS calcd for $\mathrm{C}_{37} \mathrm{H}_{38} \mathrm{~N}_{2} \mathrm{O}_{8} \mathrm{~F}_{4} \mathrm{Na}$ $\left(\mathrm{M}+\mathrm{Na}^{+}\right)$737.2462, found 737.2423 


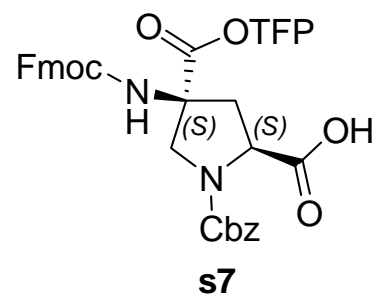

(2S,4S)-5-(9H-Fluoren-9-ylmethoxycarbonylamino)-proline-1,2,4-tricarboxylic acid

\section{1-benzyl ester 4- (2,2,3,3-tetrafluoro) propyl ester (s7)}

$9.7 \mathrm{~g}$ (13.84 $\mathrm{mmol})$ of $\mathbf{s 5}$ yielded $8.55 \mathrm{~g}(13.26 \mathrm{mmol}, 96 \%)$ of $\mathbf{s} 7$ as a white foamy solid according to the procedure given above for $\mathbf{6 a}:[\alpha]_{\mathrm{D}}^{23}=-30.0^{\circ}\left(c 0.81, \mathrm{CHCl}_{3}\right)$; IR (film) $v_{\max } 3306,3034,1713,1526,1450,1422,1260,1193,1109,759,742,697 \mathrm{~cm}^{-1} ;{ }^{1} \mathrm{H}$ NMR $\left(300 \mathrm{MHz}, 77{ }^{\circ} \mathrm{C}, \mathrm{DMSO}-d_{6}\right) \delta 8.26(\mathrm{~s}, 1 \mathrm{H}), 7.85(\mathrm{~d}, J=7.5 \mathrm{~Hz}, 2 \mathrm{H}), 7.66(\mathrm{~d}, J=7.5 \mathrm{~Hz}$, 2H), 7.31-7.40 (m, 9H), $6.40\left(\mathrm{tt}, J=52.2 \mathrm{~Hz}, J=5.1 \mathrm{~Hz},-\mathrm{CF}_{2} \mathrm{CF}_{2} \mathrm{H}, 1 \mathrm{H}\right) 5.09$ (br s, 2H), $4.57\left(\mathrm{t}, J=13.8 \mathrm{~Hz},-\mathrm{CH}_{2} \mathrm{CF}_{2^{-}}, 2 \mathrm{H}\right), 4.33-4.36(\mathrm{~m}, 3 \mathrm{H}), 4.20(\mathrm{t}, J=6.6 \mathrm{~Hz}, 1 \mathrm{H}), 4.31(\mathrm{~d}$, $J=11.4 \mathrm{~Hz}, 1 \mathrm{H}), 3.57(\mathrm{~d}, J=11.4 \mathrm{~Hz}, 1 \mathrm{H}), 2.85(\mathrm{br}, 1 \mathrm{H}), 2.31(\mathrm{br}, 1 \mathrm{H}) ;{ }^{13} \mathrm{C} \mathrm{NMR}(75.4$ $\left.\mathrm{MHz}, 20^{\circ} \mathrm{C}, \mathrm{DMSO}-d_{6}\right)$ : mixture of rotamers $\delta 172.5,172.1,170.3,155.7,153.5,153.3$, $143.5,140.6,136.5,128.2,128.1,127.7,127.5,127.3,126.9,125.0,119.9,109.0\left(t t,{ }^{1} J=\right.$ $\left.248 \mathrm{~Hz},{ }^{2} \mathrm{~J}=34 \mathrm{~Hz}, \mathrm{CH}\right), 66.2\left(\mathrm{CH}_{2}\right), 65.8\left(\mathrm{CH}_{2}\right), 62.8$ and 62.0, 60.2 (t, ${ }^{2} \mathrm{~J}=28 \mathrm{~Hz}$, $\left.\mathrm{CH}_{2}\right), 57.6$ and $57.3(\mathrm{CH}), 54.6$ and $54.2\left(\mathrm{CH}_{2}\right), 46.5(\mathrm{CH}), 38.8$ and $37.5\left(\mathrm{CH}_{2}\right)$; HPLCMS: $\mathrm{MeCN}(0.05 \%$ Formic acid) / water (0.1\% Formic Acid), $5 \%$ to $95 \%$ MeCN over 30 min; flow rate $0.80 \mathrm{~mL} / \mathrm{min}$; $\mathrm{UV}$ detection at $274 \mathrm{~nm}$; $\mathrm{t}_{\mathrm{R}}$ for $\mathbf{s} 7,24.75 \mathrm{~min}$; HRESIQTOFMS calcd for $\mathrm{C}_{32} \mathrm{H}_{28} \mathrm{~N}_{2} \mathrm{O}_{8} \mathrm{~F}_{4} \mathrm{Na}\left(\mathrm{M}+\mathrm{Na}^{+}\right) 667.1679$, found 667.1681. 


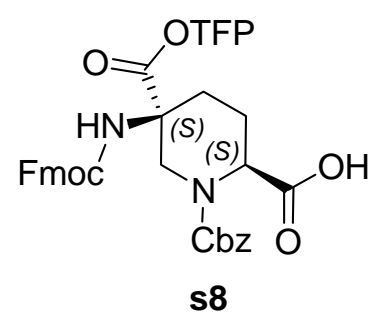

(2S,5S)-5-(9H-Fluoren-9-ylmethoxycarbonylamino)-piperidine-1,2,5-tricarboxylic acid 1-benzyl ester 5- (2,2,3,3-tetrafluoro) propyl ester (s8) $3.88 \mathrm{~g}(5.43 \mathrm{mmol})$ of $\mathbf{s 6}$ yielded $3.17 \mathrm{~g}(4.81 \mathrm{mmol}, 89 \%)$ of $\mathbf{s 8}$ as a white foamy solid according to the procedure given above for $6 \mathbf{a}:[\alpha]^{23}{ }_{\mathrm{D}}=-13.5^{\circ}\left(c 1.5, \mathrm{CHCl}_{3}\right)$; IR (film) $v_{\max } 3316,3037,1716,1523,1450,1317,1255,1118,759,742,698 \mathrm{~cm}^{-1} ;{ }^{1} \mathrm{H}$ NMR $(300$ $\left.\mathrm{MHz}, 77^{\circ} \mathrm{C}, \mathrm{DMSO}-d_{6}\right) \delta 7.86(\mathrm{~d}, J=7.2 \mathrm{~Hz}, 2 \mathrm{H}), 7.81(1 \mathrm{H}), 7.66(\mathrm{~d}, J=7.2 \mathrm{~Hz}, 2 \mathrm{H})$, 7.30-7.43 (m, 9H), 6.37 (tt, $\left.J=52.2,5.1 \mathrm{~Hz},-\mathrm{CF}_{2} \mathrm{CF}_{2} H, 1 \mathrm{H}\right) 5.11(\mathrm{br} \mathrm{s}, 2 \mathrm{H}), 4.42-4.72$ (m, 4H), $4.33(\mathrm{~d}, J=6.6 \mathrm{~Hz}, 2 \mathrm{H}), 4.20(\mathrm{t}, J=6.6 \mathrm{~Hz}, 1 \mathrm{H}) 3.00(\mathrm{br} \mathrm{d}, J=13.2 \mathrm{~Hz}, 1 \mathrm{H})$, $2.19($ br d, $J=13.2 \mathrm{~Hz}, 1 \mathrm{H}), 2.08(\mathrm{br} \mathrm{d}, J=14.1 \mathrm{~Hz}, 1 \mathrm{H}), 1.84(\mathrm{~m}, 1 \mathrm{H}) 1.63$ (ddd, $J=$ $12.9,12.9,3.0 \mathrm{~Hz}, 1 \mathrm{H}) ;{ }^{13} \mathrm{C}$ NMR $\left(75.4 \mathrm{MHz}, 20^{\circ} \mathrm{C}\right.$, DMSO- $\left.d_{6}\right)$ : mixture of rotamers $\delta$ 172.0, 170.0, 155.1, 154.7, 143.6, 143.5 and 143.4, 140.6, 136.7 and 136.5, 128.2, 127.7, 127.5, 127.1, 127.0, 126.9, 124.9, 120.0, 79.1, $66.4\left(\mathrm{CH}_{2}\right), 65.6\left(\mathrm{CH}_{2}\right), 59.7\left(\mathrm{CH}_{2}\right), 56.3$ and 56.2, 53.0 and $52.7(\mathrm{CH}), 46.5(\mathrm{CH}), 46.4\left(\mathrm{CH}_{2}\right), 28.9\left(\mathrm{CH}_{2}\right), 22.1\left(\mathrm{CH}_{2}\right)$; HPLCMS: $\mathrm{MeCN}$ ( $0.05 \%$ Formic acid) / water ( $0.1 \%$ Formic Acid), $5 \%$ to $95 \% \mathrm{MeCN}$ over 30 $\mathrm{min}$; flow rate $0.80 \mathrm{~mL} / \mathrm{min}$; UV detection at $274 \mathrm{~nm}$; $\mathrm{t}_{\mathrm{R}}$ for $\mathbf{8 8}, 25.47 \mathrm{~min}$; HRESIQTOFMS calcd for $\mathrm{C}_{32} \mathrm{H}_{28} \mathrm{~N}_{2} \mathrm{O}_{8} \mathrm{~F}_{4} \mathrm{Na}\left(\mathrm{M}+\mathrm{Na}^{+}\right)$681.1836, found 681.1802. 


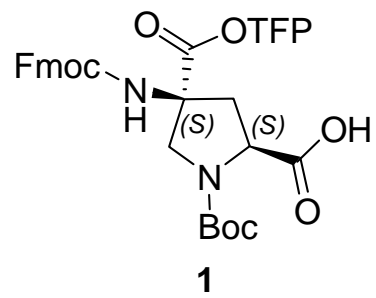

\section{(2S,4S)-5-(9H-Fluoren-9-ylmethoxycarbonylamino)-proline-1,2,4-tricarboxylic acid}

\section{1- tert-butyl ester 4- (2,2,3,3-tetrafluoro) propyl ester (1)}

To a solution of s7 ( $8.4 \mathrm{~g}, 13.1 \mathrm{mmol})$ in dry THF (10 mL per mmol of substrate) in a round bottom flask was added $840 \mathrm{mg}$ of $10 \mathrm{wt} \% \mathrm{Pd} / \mathrm{C}$ (10\% by wt. of substrate) and $\mathrm{Boc}_{2} \mathrm{O}$ (8.6 g, $39.3 \mathrm{mmol}, 3$ equiv.). The reaction mixture was degassed by applying vacuum using an aspirator and backfilled with $\mathrm{H}_{2}$ via balloon. This cycle of degassing and backfilling was repeated a few times and stirring was continued under $\mathrm{H}_{2}$ atmosphere ( 1 atm.) at room temperature. The reaction progress was monitored by HPLC. In order to speed up the reaction $1.73 \mathrm{~mL}$ of DIPEA (10.8 mmol, 0.8 equiv.) was added and stirring was further continued under $\mathrm{H}_{2}$ atmosphere. After confirming the disappearance of $\mathbf{s 7}$ (about 2 days) by HPLC, the reaction mixture was filtered through filter paper under reduced pressure and residual Pd/C was washed many times with THF. The filtrates were combined and concentrated by rotary evaporation and the resulting crude product was purified by $\mathrm{FC}\left(120 \mathrm{~g}\right.$ silica gel column, $0-10 \% \mathrm{CHCl}_{3}$ in $\mathrm{MeOH}$ gradient with $0.1 \%$ acetic acid over $16 \mathrm{CVs}$ ). The fractions containing the pure compound were combined and concentrated. Any residual acetic acid was azeotropically removed with hexane by rotary evaporation. The residual solvent was removed by applying high vacuum to yield 1 (7.2 g, $11.8 \mathrm{mmol}, 90 \%)$ as a white foamy solid: $[\alpha]^{23}{ }_{\mathrm{D}}=-54.3^{\circ}(c$ 1.35, $\mathrm{CHCl}_{3}$ ); IR (film) $v_{\max } 3304,2980,1760,1724,1530,1451,1409,1369,1259$, $1111,759,742 \mathrm{~cm}^{-1} ;{ }^{1} \mathrm{H}$ NMR $\left(300 \mathrm{MHz}, 77^{\circ} \mathrm{C}\right.$, DMSO- $\left.d_{6}\right) \delta 8.07$ (br s, $\left.1 \mathrm{H}\right), 7.85(\mathrm{~d}, J$ 
$=7.5 \mathrm{~Hz}, 2 \mathrm{H}), 7.66(\mathrm{~d}, J=7.5 \mathrm{~Hz}, 2 \mathrm{H}), 7.32-7.41(\mathrm{~m}, 4 \mathrm{H}), 6.40(\mathrm{tt}, J=52.2 \mathrm{~Hz}, J=5.1$ $\left.\mathrm{Hz},-\mathrm{CF}_{2} \mathrm{CF}_{2} \mathrm{H}, 1 \mathrm{H}\right), 4.57\left(\mathrm{t}, J=13.8 \mathrm{~Hz},-\mathrm{CH}_{2} \mathrm{CF}_{2^{-}}, 2 \mathrm{H}\right), 4.32-4.37(\mathrm{~m}, 2 \mathrm{H}), 4.18-4.23$ (m, 2H), $4.03(\mathrm{~d}, J=11.4 \mathrm{~Hz}, 1 \mathrm{H}), 3.47(\mathrm{~d}, J=11.4 \mathrm{~Hz}, 1 \mathrm{H}), 2.82(\mathrm{br}, 1 \mathrm{H}), 2.20(\mathrm{br}, 1 \mathrm{H})$, 1.38 (br s, 9H); ${ }^{13} \mathrm{C}$ NMR $\left(75.4 \mathrm{MHz}, 20{ }^{\circ} \mathrm{C}\right.$, DMSO- $\left.d_{6}\right)$ : mixture of rotamers $\delta 172.8$, $172.5,170.5,155.7,152.9,152.5,143.5,140.6,127.5,126.9,124.9,119.9,109.0\left(t t,{ }^{1} J=\right.$ $\left.248 \mathrm{~Hz},{ }^{2} J=34 \mathrm{~Hz}, \mathrm{CH}\right), 79.2,65.7\left(\mathrm{CH}_{2}\right), 62.7$ and 61.9, $60.1\left(\mathrm{t},{ }^{2} J=28 \mathrm{~Hz}, \mathrm{CH}_{2}\right), 57.5$ and 57.2 $(\mathrm{CH}), 54.4$ and 54.1 $\left(\mathrm{CH}_{2}\right), 46.5(\mathrm{CH}), 39.0$ and $37.6\left(\mathrm{CH}_{2}\right), 27.7\left(\mathrm{CH}_{3}, 3 \mathrm{C}\right)$; HPLC-MS: $\mathrm{MeCN}(0.05 \%$ Formic acid) / water (0.1\% Formic Acid), $5 \%$ to $95 \% \mathrm{MeCN}$ over $30 \mathrm{~min}$; flow rate $0.80 \mathrm{~mL} / \mathrm{min}$; $\mathrm{UV}$ detection at $274 \mathrm{~nm}$; $\mathrm{t}_{\mathrm{R}}$ for $\mathbf{1}, 24.21 \mathrm{~min}$; HRESIQTOFMS calcd for $\mathrm{C}_{29} \mathrm{H}_{30} \mathrm{~N}_{2} \mathrm{O}_{8} \mathrm{~F}_{4} \mathrm{Na}\left(\mathrm{M}+\mathrm{Na}^{+}\right)$633.1836, found 633.1835.

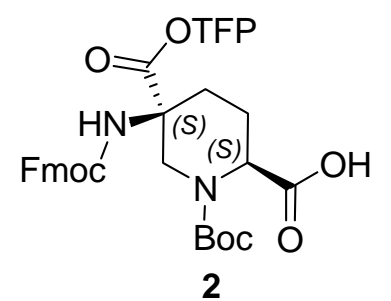

(2S,5S)-5-(9H-Fluoren-9-ylmethoxycarbonylamino)-piperidine-1,2,5-tricarboxylic acid 1- tert-butyl ester 5- (2,2,3,3-tetrafluoro) propyl ester (2) $3.07 \mathrm{~g}(4.66 \mathrm{mmol})$ of $\mathbf{s 8}$ yielded $2.03 \mathrm{~g}(3.25 \mathrm{mmol}, 70 \%)$ of $\mathbf{2}$ as a white foamy solid according to the procedure given above for $\mathbf{1}:[\alpha]^{23}{ }_{\mathrm{D}}=-12.5^{\circ}\left(\mathrm{c} 1.26, \mathrm{CHCl}_{3}\right)$; IR (film) $v_{\max } 3316,2978,1716,1524,1450,1420,1369,1319,1255,1118,759,741 \mathrm{~cm}^{-1} ;{ }^{1} \mathrm{H}$ NMR $\left(300 \mathrm{MHz}, 77^{\circ} \mathrm{C}, \mathrm{DMSO}-d_{6}\right) \delta 7.86(\mathrm{~d}, J=7.5 \mathrm{~Hz}, 2 \mathrm{H}), 7.77(\mathrm{~s}, 1 \mathrm{H}), 7.67(\mathrm{~d}, J=$ 7.2 Hz, 2H), 7.30-7.44 (m, 4H), $6.39\left(\mathrm{tt}, J=52.2,5.1 \mathrm{~Hz},-\mathrm{CF}_{2} \mathrm{CF}_{2} \mathrm{H}, 1 \mathrm{H}\right), 4.46-4.58(\mathrm{~m}$, 4H), $4.32(\mathrm{~d}, J=6.6 \mathrm{~Hz}, 2 \mathrm{H}), 4.33(\mathrm{t}, J=6.6 \mathrm{~Hz}, 1 \mathrm{H}) 2.94(\mathrm{br} \mathrm{d}, J=13.5 \mathrm{~Hz}, 1 \mathrm{H}), 2.19$ (br d, $J=13.8 \mathrm{~Hz}, 1 \mathrm{H}), 1.99-2.07(\mathrm{~m}, 1 \mathrm{H}), 1.86(\mathrm{~m}, 1 \mathrm{H}) 1.63(\mathrm{ddd}, J=12.9,12.9,3.6$ $\mathrm{Hz}, 1 \mathrm{H}), 1.40(\mathrm{~s}, 9 \mathrm{H}) ;{ }^{13} \mathrm{C}$ NMR $\left(75.4 \mathrm{MHz}, 20{ }^{\circ} \mathrm{C}\right.$, DMSO- $\left.d_{6}\right)$ : mixture of rotamers $\delta$ 
172.4, 170.2, 170.1, 155.2, 154.1, 143.6, 140.7, 127.6, 127.0, 125.0, 120.1, 79.4, 79.1, 65.6 $\left(\mathrm{CH}_{2}\right), 59.8$ and $59.7\left(\mathrm{CH}_{2}\right), 56.4$ and 56.3, $53.4(\mathrm{CH}), 46.5(\mathrm{CH}), 45.8\left(\mathrm{CH}_{2}\right), 29.2$ $\left(\mathrm{CH}_{2}\right), 27.8\left(\mathrm{CH}_{3}, 3 \mathrm{C}\right), 22.2\left(\mathrm{CH}_{2}\right)$; HPLC-MS: $\mathrm{MeCN}(0.05 \%$ Formic acid) / water (0.1\% Formic Acid), $5 \%$ to $95 \% \mathrm{MeCN}$ over $30 \mathrm{~min}$; flow rate $0.80 \mathrm{~mL} / \mathrm{min}$; UV detection at $274 \mathrm{~nm}$; $t_{\mathrm{R}}$ for $\mathbf{2}, 25.23 \mathrm{~min}$; HRESIQTOFMS calcd for $\mathrm{C}_{30} \mathrm{H}_{32} \mathrm{~N}_{2} \mathrm{O}_{8} \mathrm{~F}_{4} \mathrm{Na}$ (M $+\mathrm{Na}^{+}$) 647.1992, found 647.2028.

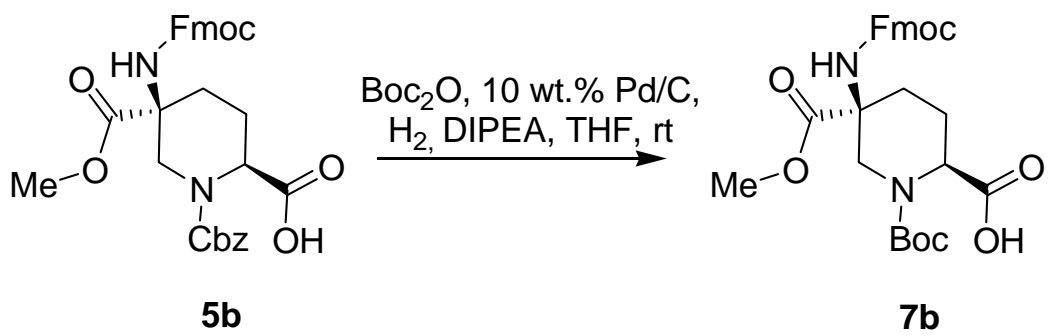

Supplemental Scheme 3: Synthesis of monomer 7b

\section{(2S,5S)-5-(9H-Fluoren-9-ylmethoxycarbonylamino)-piperidine-1,2,5-tricarboxylic}

\section{acid 1- tert-butyl ester 5- methyl ester (7b)}

$280 \mathrm{mg}(0.5 \mathrm{mmol})$ of $\mathbf{5} \mathbf{b}^{2}$ yielded $198 \mathrm{mg}(0.38 \mathrm{mmol}, 76 \%)$ of $\mathbf{7 b}$ as a white foamy solid according to the procedure given above for $\mathbf{1}:[\alpha]^{23}{ }_{\mathrm{D}}=-17.8^{\circ}\left(c 0.5, \mathrm{CHCl}_{3}\right) ; \mathrm{IR}$ (film) $v_{\max } 3316,2977,1716,1525,1450,1421,1369,1319,1255,1118,759,741 \mathrm{~cm}^{-1}$; ${ }^{1} \mathrm{H}$ NMR $\left(300 \mathrm{MHz}, 77{ }^{\circ} \mathrm{C}\right.$, DMSO- $\left.d_{6}\right) 7.86(\mathrm{~d}, J=7.2 \mathrm{~Hz}, 2 \mathrm{H}), 7.68(\mathrm{~d}, J=7.2 \mathrm{~Hz}, 2 \mathrm{H})$, $7.58(\mathrm{~s}, 1 \mathrm{H}), 7.30-7.44(\mathrm{~m}, 4 \mathrm{H}), 4.59(\mathrm{~m}, 1 \mathrm{H}), 4.49(\mathrm{~d}, J=13.5 \mathrm{~Hz}, 1 \mathrm{H}), 4.32(\mathrm{~d}, J=6.6$ $\mathrm{Hz}, 2 \mathrm{H}), 4.20(\mathrm{t}, J=6.6 \mathrm{~Hz}, 1 \mathrm{H}), 3.59$ (br s, 3H), $2.95(\mathrm{~d}, J=13.5 \mathrm{~Hz}, 1 \mathrm{H}), 2.20$ (br d, $J$ $=13.5 \mathrm{~Hz}, 1 \mathrm{H}), 1.81-2.02(\mathrm{~m}, 2 \mathrm{H}), 1.54(\mathrm{ddd}, J=12.6,12.6,4.2 \mathrm{~Hz}, 1 \mathrm{H}) ;{ }^{13} \mathrm{C}$ NMR $(75.4$ $\mathrm{MHz}, 2{ }^{\circ} \mathrm{C}$, DMSO- $\left.d_{6}\right)$ : mixture of rotamers $\delta 172.2,171.7,154.9,153.9,143.6,140.6$, 127.5, 126.9, 125.0, 120.0, 79.4, 79.2, $65.4\left(\mathrm{CH}_{2}\right), 56.2,52.1(\mathrm{CH}), 51.8\left(\mathrm{CH}_{3}\right), 46.5$ $(\mathrm{CH}), 45.9\left(\mathrm{CH}_{2}\right), 29.2\left(\mathrm{CH}_{2}\right), 27.8\left(\mathrm{CH}_{3}, 3 \mathrm{C}\right), 22.3\left(\mathrm{CH}_{2}\right)$; HPLC-MS: MeCN $(0.05 \%$ 
Formic acid) / water (0.1\% Formic Acid), 5\% to 95\% MeCN over 30 min; flow rate 0.80 $\mathrm{mL} / \mathrm{min}$; UV detection at $274 \mathrm{~nm}$; $\mathrm{t}_{\mathrm{R}}$ for $\mathbf{7 b}, 22.91 \mathrm{~min}$; HRESIQTOFMS calcd for $\mathrm{C}_{28} \mathrm{H}_{32} \mathrm{~N}_{2} \mathrm{O}_{8} \mathrm{Na}\left(\mathrm{M}+\mathrm{Na}^{+}\right)$547.2056, found 547.2066. 


\section{Measurement of kinetics of diketopiperazine formation}

\begin{tabular}{|c|c|c|c|}
\hline $\begin{array}{l}\text { (i) Fmoc-L-Tyr }(t-\mathrm{Bu}) \\
\text { HATU,DIPEA }\end{array}$ & (i) A, HATU,DIPEA & (i) $\mathbf{B}, \mathrm{HATU}$,DIPEA & (i) $10 \% \mathrm{Ac}_{2} \mathrm{O} /$ Pyridine \\
\hline
\end{tabular}
MBHA resin

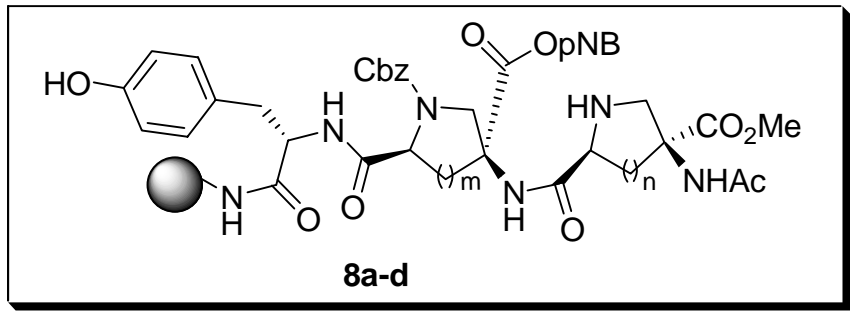

$$
\begin{array}{ll}
8 \mathbf{a}(m=1, n=1) & \mathbf{A}=6 \mathbf{a} \quad \mathbf{B}=7 \mathbf{a}, \\
8 \mathbf{b}(m=1, n=2) & \mathbf{A}=6 \mathbf{a} \quad \mathbf{B}=7 \mathbf{b}, \\
8 \mathbf{c}(m=2, n=1) & \mathbf{A}=6 \mathbf{b} \quad \mathbf{B}=7 \mathbf{a}, \\
8 \mathbf{d}(m=2, n=2) & \mathbf{A}=\mathbf{6 b} \quad \mathbf{B}=7 \mathbf{b}
\end{array}
$$

Supplemental Scheme 4: Assembly of bis amino acid dimers

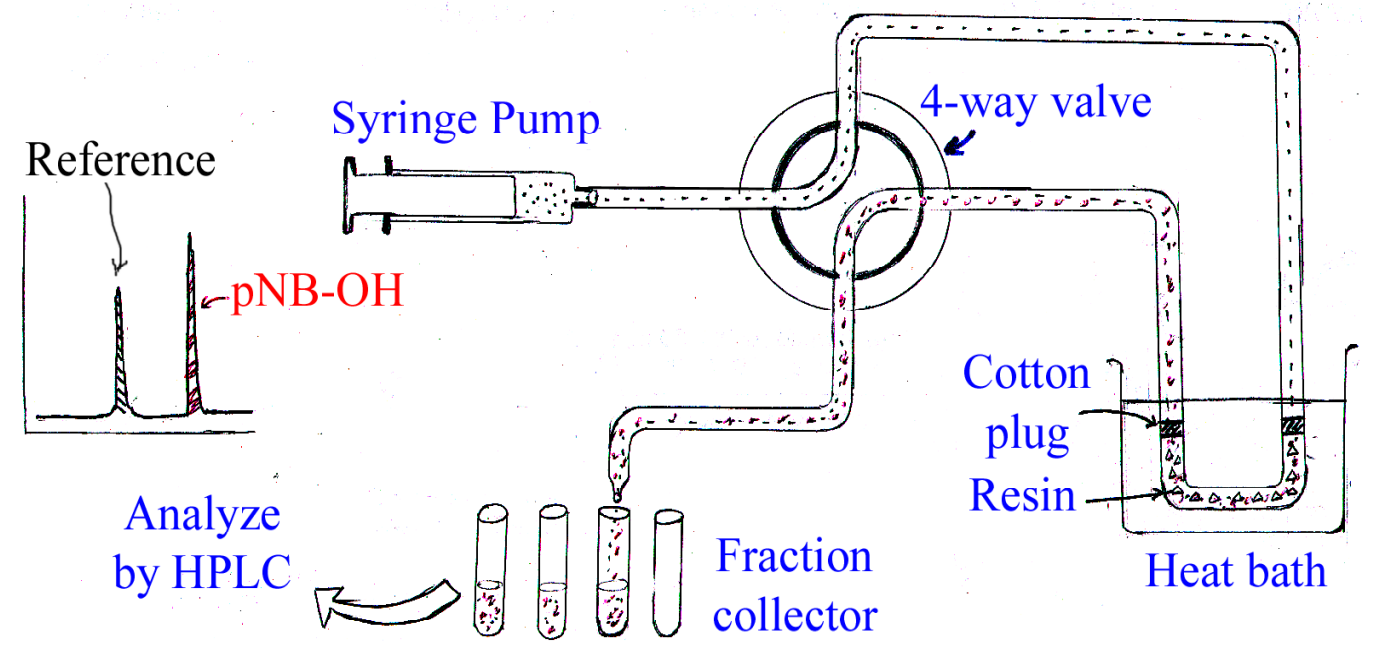

Supplemental Figure 1: Schematic Diagram of the flow cell set up for the kinetic measurements.

The above shown simple set up for the measurement of half-life for DKP closure reaction was assembled from the PTFE tubings and connectors. All the connection were made by a thin tubing with inner diameter $0.75 \mathrm{~mm}$. However for flow cell a small piece 
of tubing $(15 \mathrm{~mm})$ with inner diameter $1.55 \mathrm{~mm}$ was used. This tubing was stuffed with a few beads of resin $(2-5 \mathrm{mg})$ and they were held in their place by cotton plugs at both ends. The oil bath was maintained at desired temperature using digital temperature controller. The eluant was prepared by dissolving approximately $20 \mu \mathrm{M}$ of reference compound (9-Fluorenemethanol) and the desired amount of catalyst in the solvent. The resin was allowed to swell by pumping blank solvent through the flow cell. After a few minutes the flow cell tube carrying the resin was taken out of the loop and the system was allowed to equilibrate by pumping the eluant through. The flow cell was put back into the loop, lowered into the heat bath and flow rate was set at $100 \mu \mathrm{L}$ per minute. After 5 minutes (compensating for the dead volume of tubing from resin to collector) fractions were collected at 15 minute intervals using automatic fraction collector. $5 \mu \mathrm{L}$ from each fraction were injected into HPLC for analysis. Areas under the peaks were recorded for the reference and the alcohol and used for further calculations.

General procedure for the data analysis and calculation has been explained for one of the experiments for which $\mathbf{8 c}$ was used as the test sequence.
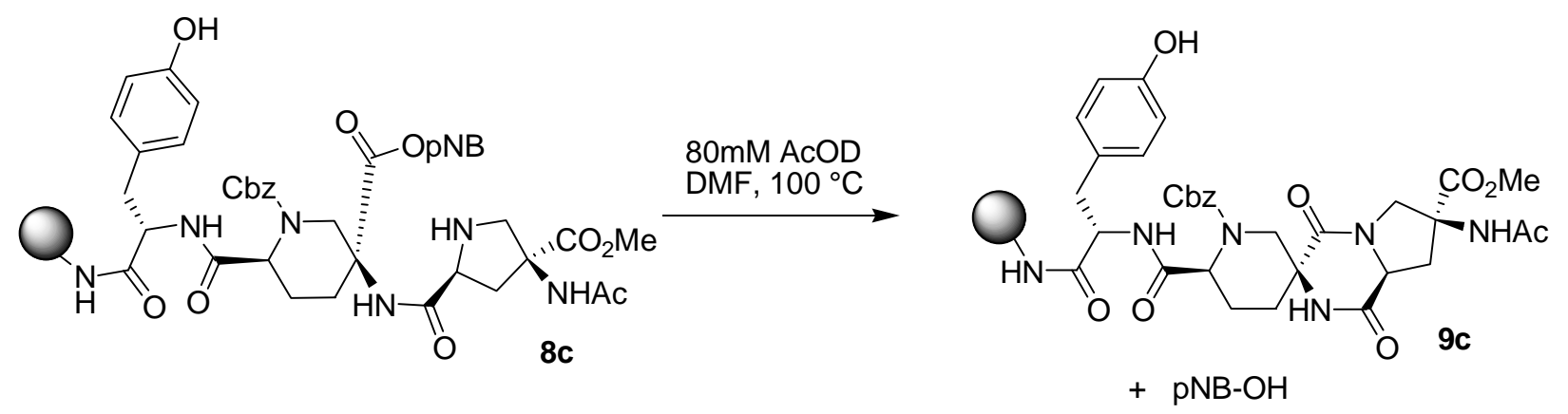

Supplemental Scheme 5: diketopiperazine formation in flow cell for sequence 8c

Overall aminolysis reaction can be represented as

$$
\mathrm{X} \rightarrow \mathrm{Y}+\mathrm{Z}
$$


Since catalyst is flowed over the resin at a fixed rate, it can be safely assumed that reaction follows first order reaction rate equation

$$
d[Y] / d t=-d[X] / d t=k[X]
$$

The quantity we are measuring in our experiments is the area under the curve (I) for the release of pNitrobenzyl alcohol (Y). Hence (I) at any time t represents amount of $\mathrm{Y}$ released for a time interval $\mathrm{t}-\Delta \mathrm{t}$ to $\mathrm{t}$.

Thus

$$
\begin{aligned}
& I=a\left\{[Y]_{t}-[Y]_{t-\Delta t}\right\} \\
& I=a\left\{[X]-[X]_{t}-[X]+[X]_{t-\Delta t}\right\} \\
& I=a\left\{[X]_{t-\Delta t}-[X]_{t}\right\}
\end{aligned}
$$

However general solution for eq. 1 is given by

$$
\begin{aligned}
& {[X]_{t}=[X]_{0} e^{-k t}} \\
& \Rightarrow I=a[X]_{0}\left\{e^{-k(t-\Delta t)}-e^{-k t}\right\} \\
& \Rightarrow I=a[X]_{0}\left(e^{k \Delta t}-1\right) e^{-k t}
\end{aligned}
$$

$\Rightarrow I=A e^{-k t} \quad$ where $\mathrm{A}=a[X]_{0}\left(e^{k \Delta t}-1\right)$, is constant as long as $\Delta \mathrm{t}$ is a constant

$$
\Rightarrow \ln I=-k t+\ln A
$$

Therefore if the plot lnI against time $t$ gives a straight line, than the aminolysis reaction does follow first order reaction rate equation and halflife for this transformation is given by

$$
t_{1 / 2}=\ln 2 / k=0.693 / k
$$


Since $[\mathrm{X}]_{0}$ appears as a constant it can be chosen arbitrarily without affecting the calculation of half life. Also we normalized the area under the curve for $p$-nitrobenzyl alcohol (I) by dividing it by the area under the curve for reference, $\mathrm{I}_{\mathrm{ref}}$ to remove any discrepancies arising due to instrumental errors.

$\begin{array}{lllll}\mathbf{t}(\mathbf{m i n}) & \mathbf{I}\left(\mathbf{m A} \mathbf{U}^{*} \mathbf{s e c}\right) & \mathbf{I}_{\text {ref }}\left(\mathbf{m A U} \mathbf{U}^{*} \mathbf{s e c}\right) & \mathbf{I}^{\prime}=\left(\mathbf{I} / \mathbf{I}_{\text {ref }}\right) & \mathbf{I n I} \\ 15 & 746.9 & 1640.7 & 0.455 & -0.787 \\ 30 & 1877.6 & 2451.2 & 0.766 & -0.267 \\ 45 & 896.7 & 2414.3 & 0.371 & -0.990 \\ 60 & 460.8 & 2472.4 & 0.186 & -1.68 \\ 75 & 238.7 & 2417.4 & 0.0987 & -2.315 \\ 90 & 144.2 & 2464.3 & 0.0585 & -2.838\end{array}$

Supplementary Table 1: Data obtained for on resin DKP closure for sequence 6c

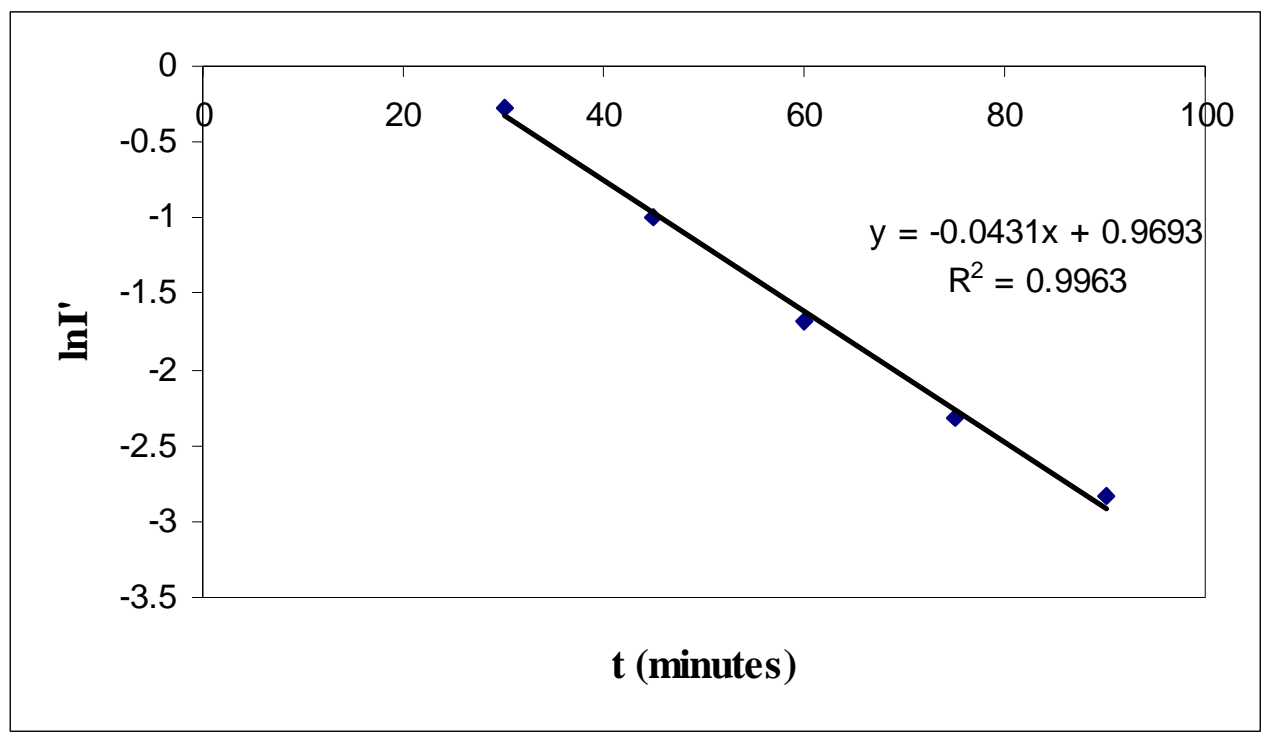

Supplementary figure 2: Graph of $\ln \left(I^{\prime}\right)$ Vs. time $t$

From graph, $\mathrm{k}=0.0431 \mathrm{~min}^{-1}$

Hence half life, $\quad t_{1 / 2}=0.693 / k$

$$
\mathrm{t}_{1 / 2}=0.693 / 0.0431=16.1 \text { minute }
$$


In order to make sure that the reaction did go to completion and to look for the evidence of epimerization, resin was recovered from the tubing and cleaved using TFMSA cleavage method similar to the one described in Novabiochem catalogue (see experimental section in manuscript for detail).
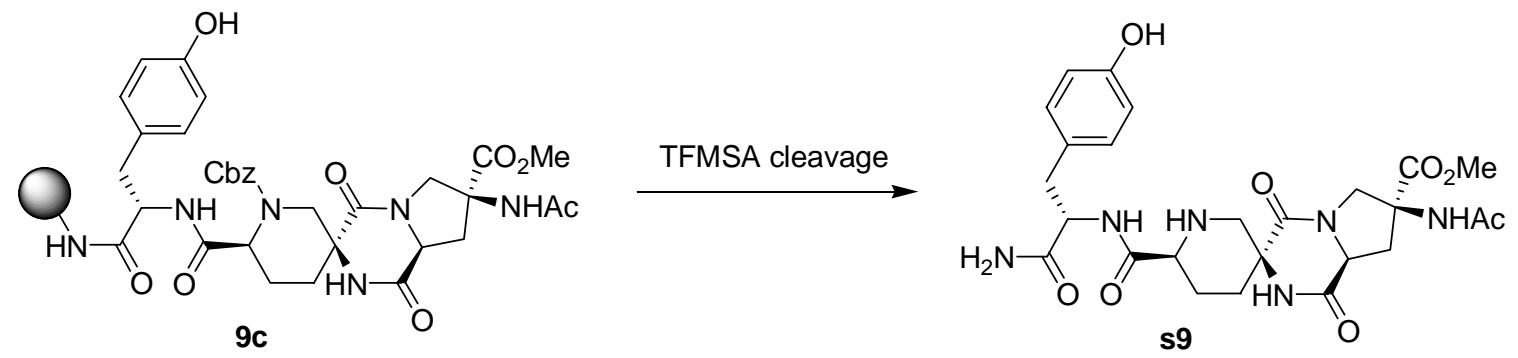

Supplemental Scheme 6: Cleavage of the product s9 following the DKP formation reaction

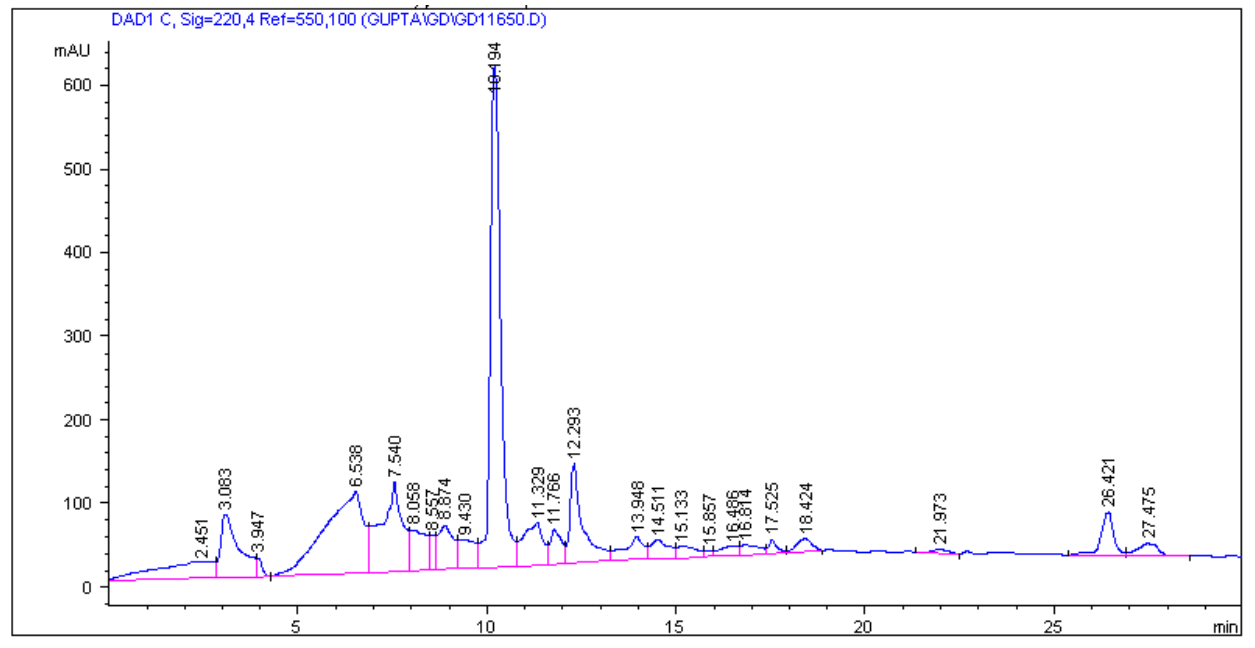

Supplemental Figure 3: Chromatogram of the cleaved product s7 at $220 \mathrm{~nm}$.

HPLC-MS: column, Waters XTerra MS $\mathrm{C}_{18}$ column $4.6 \mathrm{~mm}$ x $150 \mathrm{~mm}$; mobile phase, $\operatorname{MeCN}(0.05 \%$ Formic acid) / water (0.1\% Formic Acid), $5 \%$ to $95 \% \mathrm{MeCN}$ over $30 \mathrm{~min}$; flow rate $0.80 \mathrm{~mL} / \mathrm{min}$; UV detection at $220 \mathrm{~nm}$; $\mathrm{t}_{\mathrm{R}}$ for $\mathbf{s 9}, 10.194 \mathrm{~min}$; ES-LRMS m/z (ion), calculated 545.2( $\left.\mathrm{M}+\mathrm{H}^{+}\right)$observed 545.0. 


\section{Microwave assisted DKP closure in oligomer 10}

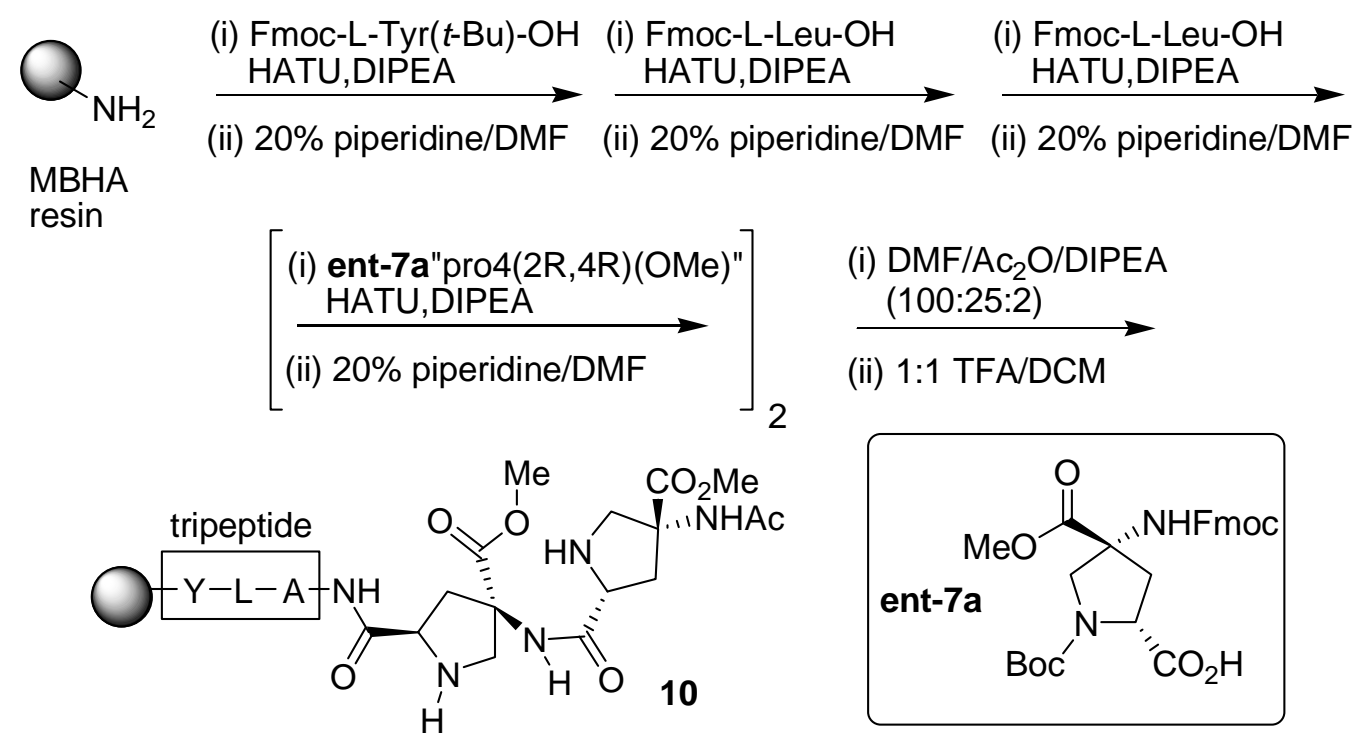

Supplemental Scheme 7: Assembly of sequence 10 on MBHA resin.

The homo oligomer 10 was synthesized on MBHA resin (100mg resin, 0.62

$\mathrm{mmol} / \mathrm{g}$ loading) following a procedure similar to the one described for 13. Accordingly, Fmoc-L-Tyr(t-Bu)-OH (43.4 mg, 94.5 mol), Fmoc-L-Leucine-(OH) $(33.4$ mg, 94.5

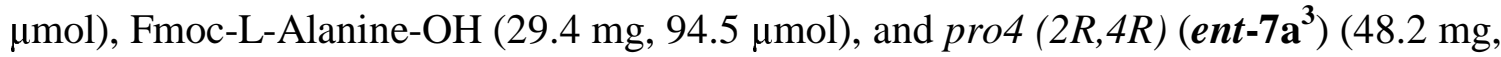
$94.5 \mu \mathrm{mol})$. were coupled sequentially to the resin using HATU as coupling agent and DIPEA as base. This was followed by removal of terminal Fmoc group to unmask the free amine and acylation by $1 \mathrm{~mL}$ of a solution of DMF, Acetic Anhydride and DIPEA (100:25:2) for 30 minutes. The resin was washed three times with DMF and $\mathrm{CH}_{2} \mathrm{Cl}_{2}$ alternately and three times with $\mathrm{MeOH}$ and $\mathrm{CH}_{2} \mathrm{Cl}_{2}$ alternately. Boc group removal was affcted by treatment of the resin with $1 \mathrm{~mL}$ of $1: 1 \mathrm{TFA} / \mathrm{DCM}(2 \times 15$ minute). The resin was washed with $\mathrm{CH}_{2} \mathrm{Cl}_{2}$ and $\mathrm{MeOH}$ many times and neutralized by washing a few times

( $\left.{ }^{3}\right)$ Levins, C. G.; Schafmeister, C. E. J. Org. Chem. 2005, 70, 9002-9008. 
with $10 \% \mathrm{Et}_{3} \mathrm{~N} / \mathrm{CH}_{2} \mathrm{Cl}_{2}$. Finally the resin was washed with DCM and MeOH many times, followed by only $\mathrm{CH}_{2} \mathrm{Cl}_{2}$ and dried under reduced pressure overnight to yield $\mathbf{1 0}$.

On the resin diketopiperazine formation in oligomer 10 via microwave irradiation.

A few beads of MBHA resin loaded with oligomer 10, Y-L-A-pro4 (2R4R)-pro4 $(2 R 4 R)$ were placed in a microwave reaction vessel (glass, $10 \mathrm{~mL}$ capacity) containing 4 $\mathrm{mL}$ of $80 \mathrm{mM}$ AcOD in DMF. The vessel was capped and placed in the microwave reactor (CEM Discover) and irradiated $\left(75 \mathrm{~W}\right.$ maximum power, $100^{\circ} \mathrm{C}, 10 \mathrm{~min}$ ramp) for desired length of time. The tube was than removed from the microwave reactor and the resin was transferred to a $1 \mathrm{~mL}$ polypropylene SPPS reactor. The resin was washed with DCM many times and placed under high vacuum overnight.

The mixture of oligomers $\mathbf{1 1}$ and $\mathbf{1 2}$ was cleaved from the resin using the TFMSA procedure as given earlier in manuscript for 14. After centrifugation residual pellet was dissolved in $200 \mu \mathrm{L}$ of 1:1 MeCN/water and $10 \mu \mathrm{L}$ of this solution were injected into HPLC for analysis.

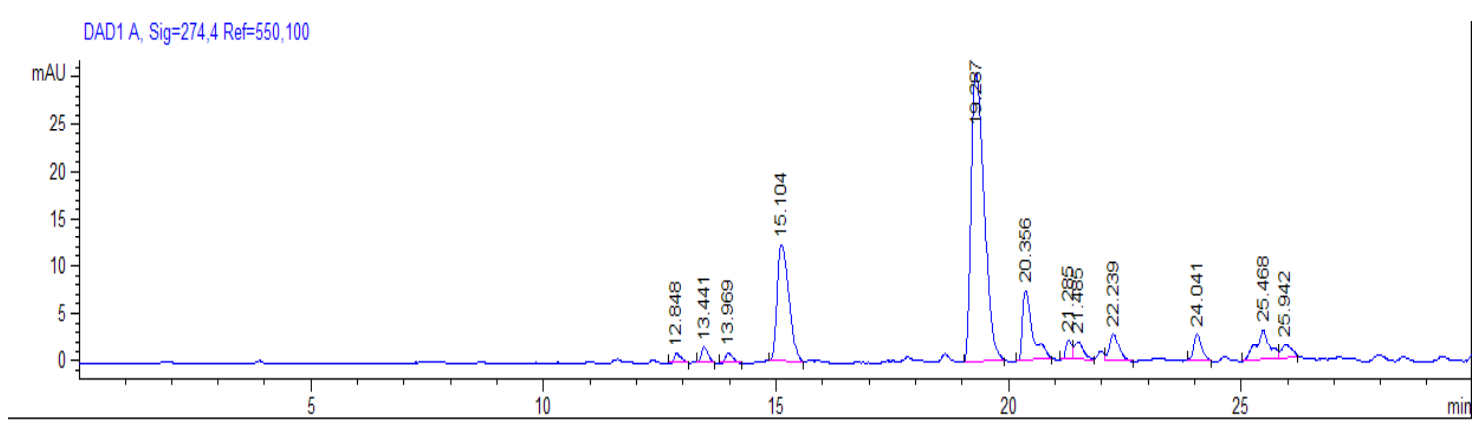

Supplemental Figure 4: A representative chromatogram for the cleaved product from diketopiperazine closure in oligomer $\mathbf{1 0}$ at $274 \mathrm{~nm}$ (60 min microwave irradiation) HPLC-MS: mobile phase, $\mathrm{MeCN}$ / water (0.1\% Formic Acid), $0 \%$ to $95 \% \mathrm{MeCN}$ over 30 min; flow rate $0.80 \mathrm{~mL} / \mathrm{min}$; $\mathrm{UV}$ detection at $274 \mathrm{~nm}$; $\mathrm{t}_{\mathrm{R}}$ for $\mathbf{1 2}, 15.10 \mathrm{~min}$; ES-LRMS 
calcd for $\mathrm{C}_{34} \mathrm{H}_{50} \mathrm{~N}_{8} \mathrm{O}_{1}\left(\mathrm{M}+\mathrm{H}^{+}\right)$746.4, observed 746.7; $\mathrm{t}_{\mathrm{R}}$ for 11, $19.29 \mathrm{~min}$; ES-LRMS calcd for $\mathrm{C}_{33} \mathrm{H}_{46} \mathrm{~N}_{8} \mathrm{O}_{1}\left(\mathrm{M}+\mathrm{H}^{+}\right)$714.3, observed 714.7.

Supplemental Table 2: Areas under the major peaks observed in chromotograms with varying microwave irradiation time $(\mathrm{t})$

\begin{tabular}{|c|c|c|c|c|}
\hline $\begin{array}{c}\text { Time for MW } \\
\text { irradiation } \\
\mathrm{t}(\mathrm{min})\end{array}$ & $\begin{array}{c}\text { Integrated Areas for } \\
\text { unreacted Starting } \\
\text { Material } \mathrm{X}\left(\mathrm{mAU} \mathrm{U}^{*}\right)\end{array}$ & $\begin{array}{c}\text { Integrated Area for } \\
\text { desired Product } \\
\mathrm{Y}\left(\mathrm{mAU}^{*} \mathrm{~s}\right)\end{array}$ & $\begin{array}{c}\text { Fraction of starting } \\
\text { material at time } \mathrm{t} \\
{[\mathrm{F}]_{\mathrm{t}}=\mathrm{X} /(\mathrm{X}+\mathrm{Y})}\end{array}$ & $\mathrm{Ln}[\mathrm{F}]_{\mathrm{t}}$ \\
\hline 5 & 643.2 & 197.0 & 0.755 & -0.281 \\
\hline 15 & 401.6 & 240.3 & 0.626 & -0.468 \\
\hline 30 & 116.1 & 116.1 & 0.500 & -0.693 \\
\hline 45 & 537.0 & 1016.6 & 0.346 & -1.061 \\
\hline 60 & 216.4 & 595.0 & 0.267 & -1.321
\end{tabular}

\section{Analysis of Data:}

The aminolysis reaction can be represented as $\mathrm{X} \rightarrow \mathrm{Y}+\mathrm{Z}$ and the reaction simply follows first order rate equation;

$$
-d[X] / d t=k^{\prime}[X]
$$


General solution for eq. 2 is given by

$[X]_{t}=[X]_{0} e^{-k t}$

$\Rightarrow[X]_{t} /[X]_{0}=e^{-k t}$

$\Rightarrow[X]_{t} /\left([X]_{t}+[Y]_{t}\right)=e^{-k t}$

$\Rightarrow[F]_{t}=e^{-k t}$ where $[\mathrm{F}]_{t}$ represents fraction of reactant present at time $\mathrm{t}$

$\Rightarrow \ln [F]_{t}=-k t$

Therefore if the plot $\ln [F]_{t}$ against time $t$ gives a straight line, than the aminolysis reaction does follow first order reaction rate equation and halflife for this transformation is given by

$t_{1 / 2}=\ln 2 / k=0.693 / k$

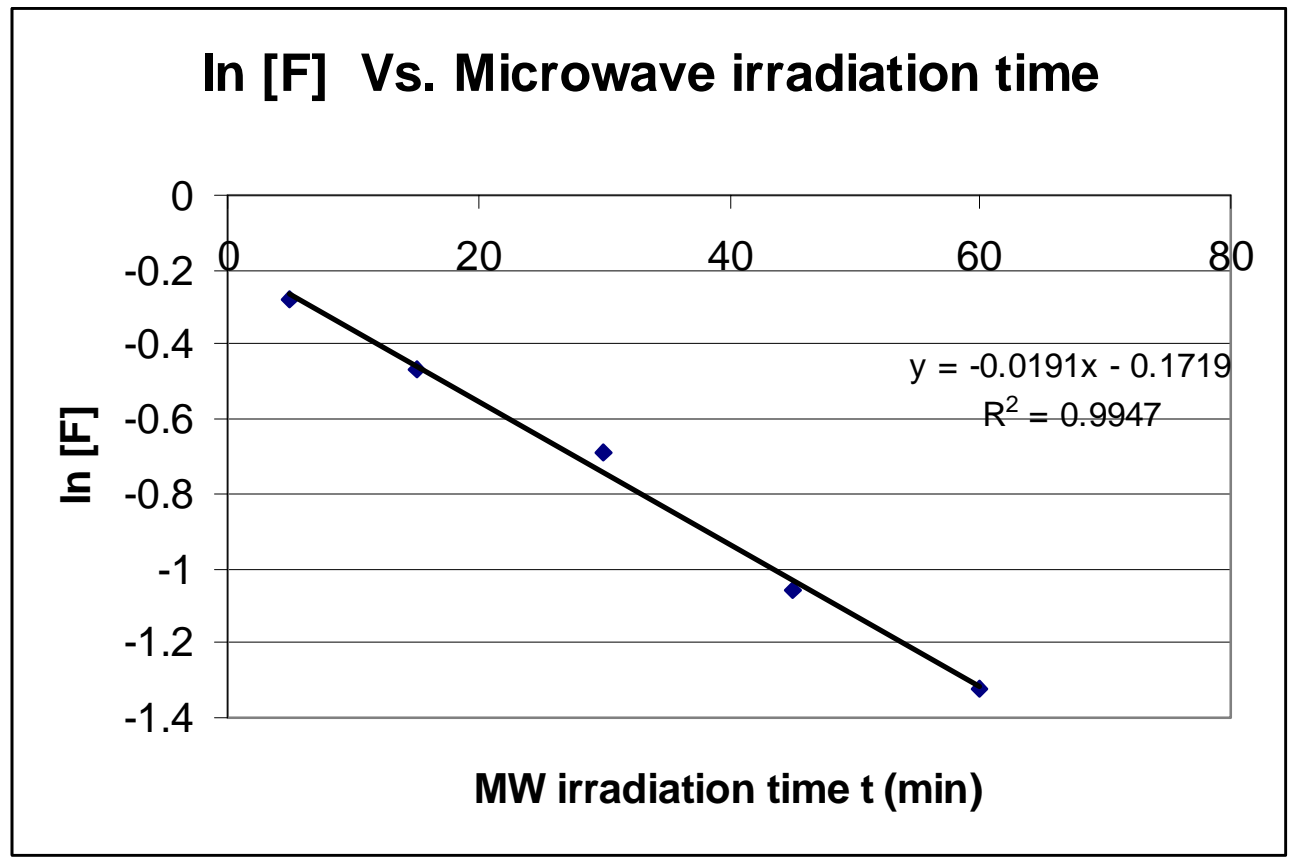

Supplementary Figure 5: Plot of $\ln (\mathrm{F})$ against MW irradiation time (t).

From graph, $\quad \mathrm{k}=0.0191 \mathrm{~min}^{-1}$

Hence half life, $\mathrm{t}_{1 / 2}=0.693 / \mathrm{k}=0.693 / 0.0191=36.3$ minute 


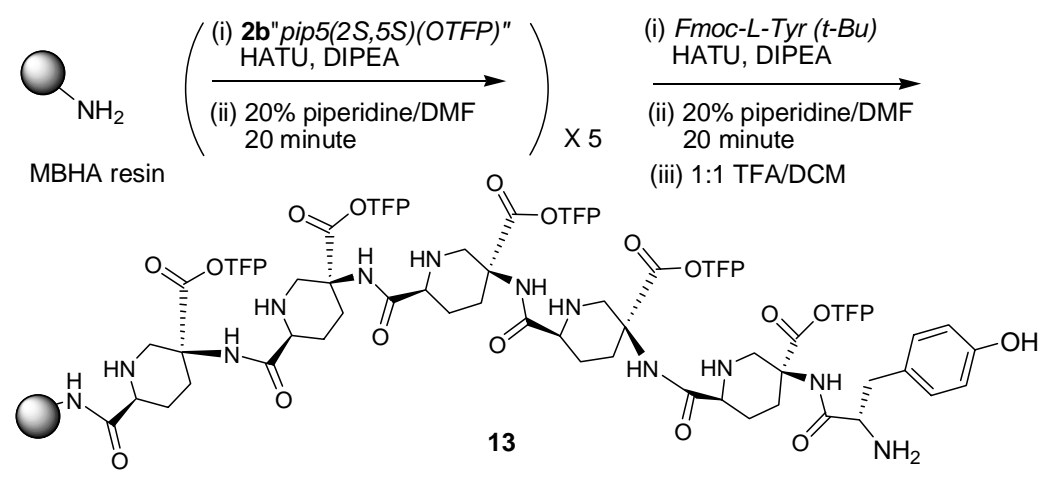

Supplemental Scheme 8: Assembly of 5-mer homosequence 13 on MBHA resin.

\section{HPLC chromotograms for the oligomers before purification.}

For all the chromotograms: column, Waters XTerra MS $C_{18}$ column $4.6 \mathrm{~mm}$ x $150 \mathrm{~mm}$; mobile phase, $\operatorname{MeCN}(0.05 \%$ Formic acid $) /$ water ( $0.1 \%$ Formic Acid), $0 \%$ to $25 \%$ MeCN over $30 \mathrm{~min}$; flow rate $0.80 \mathrm{~mL} / \mathrm{min}$; UV detection at $220 \mathrm{~nm}$;

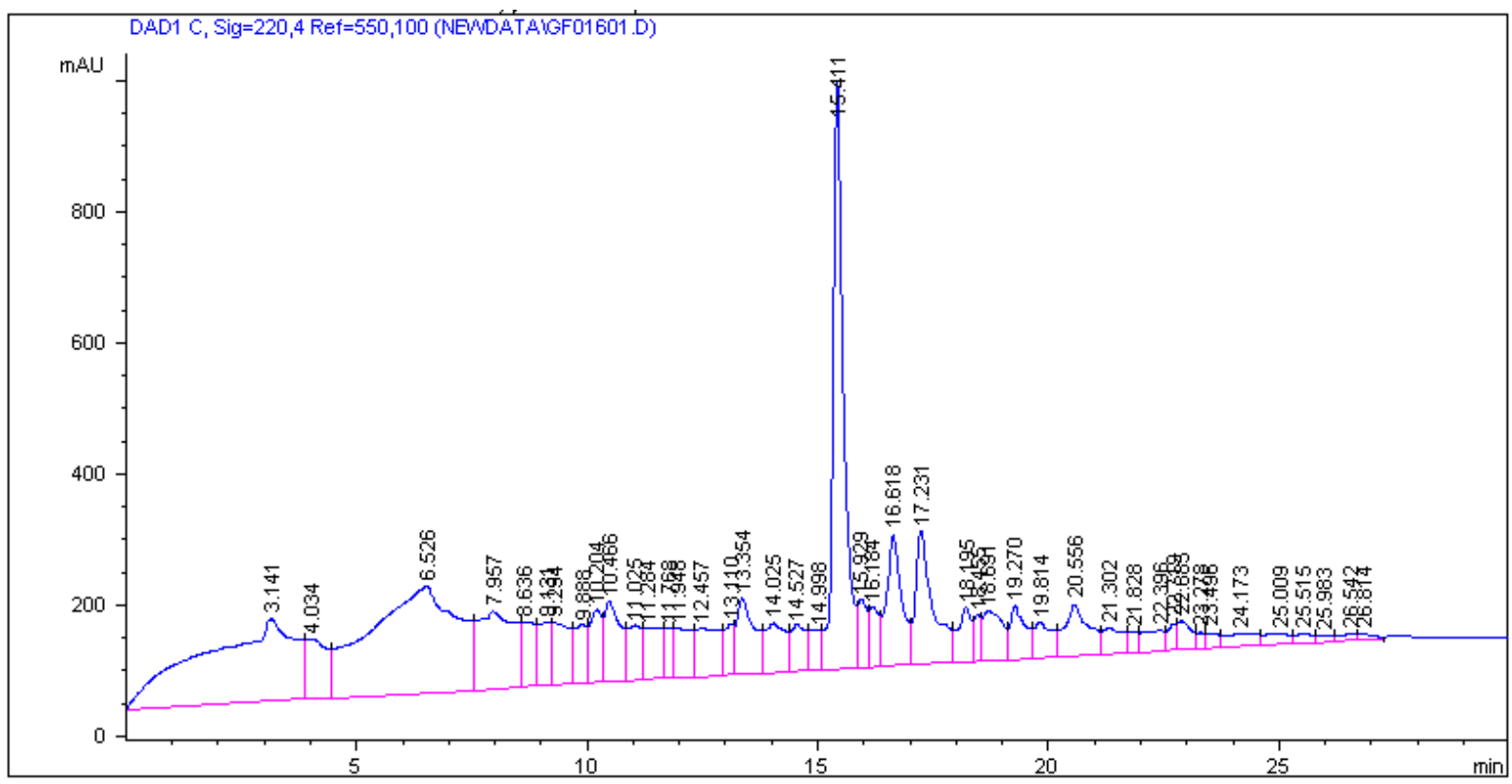

Supplemental Figure 6: HPLC chromatogram for homo-oligomer 14 at $220 \mathrm{~nm}$

HPLC-MS: $t_{\mathrm{R}}$ for 14, $15.41 \mathrm{~min}$; ES-LRMS calcd for $\mathrm{C}_{44} \mathrm{H}_{53} \mathrm{~N}_{12} \mathrm{O}_{12}\left(\mathrm{M}+\mathrm{H}^{+}\right)$941.4, found 941.2; 


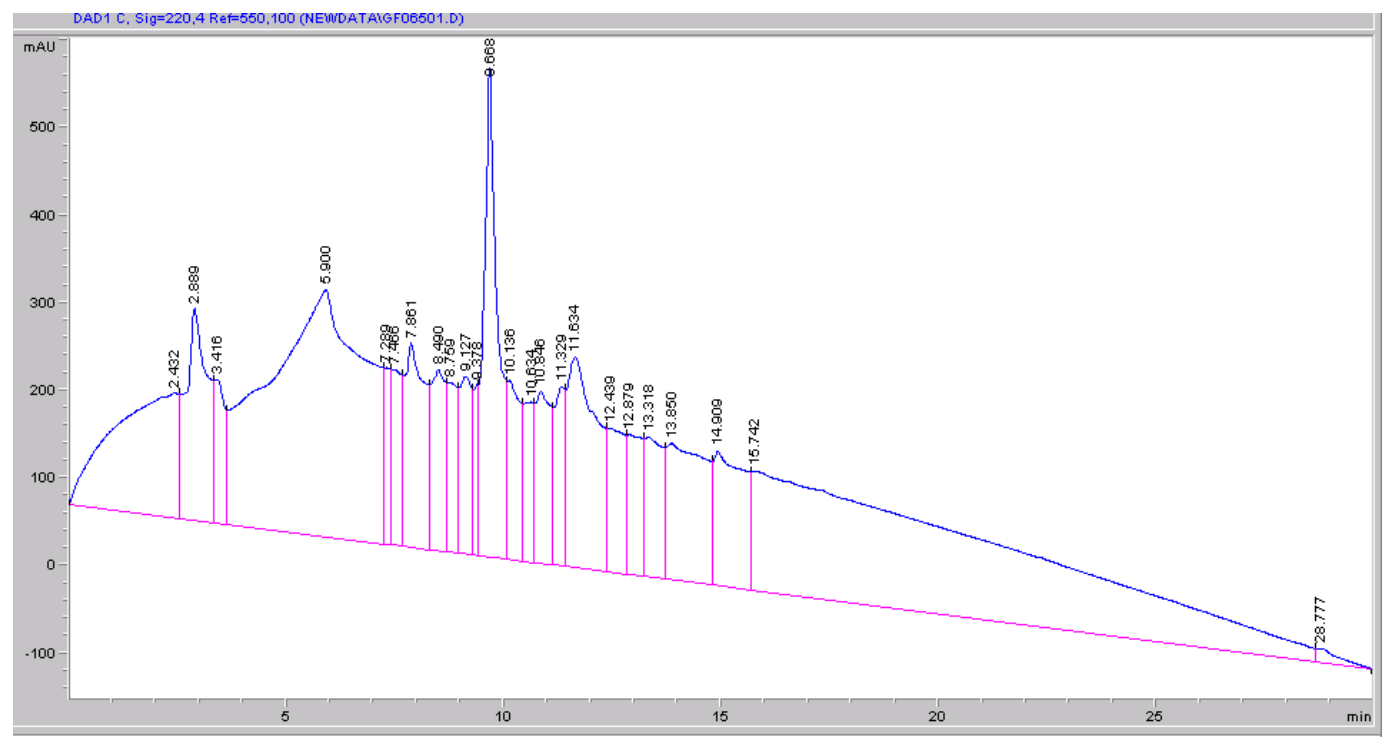

Supplemental Figure 7: HPLC chromatogram for hetero-oligomer 3 at $220 \mathrm{~nm}$ HPLC-MS: $t_{R}$ for 3, 9.7 min; ES-LRMS calcd for $\mathrm{C}_{35} \mathrm{H}_{40} \mathrm{~N}_{9} \mathrm{O}_{11}\left(\mathrm{M}+\mathrm{H}^{+}\right) 762.3$, found 762.2

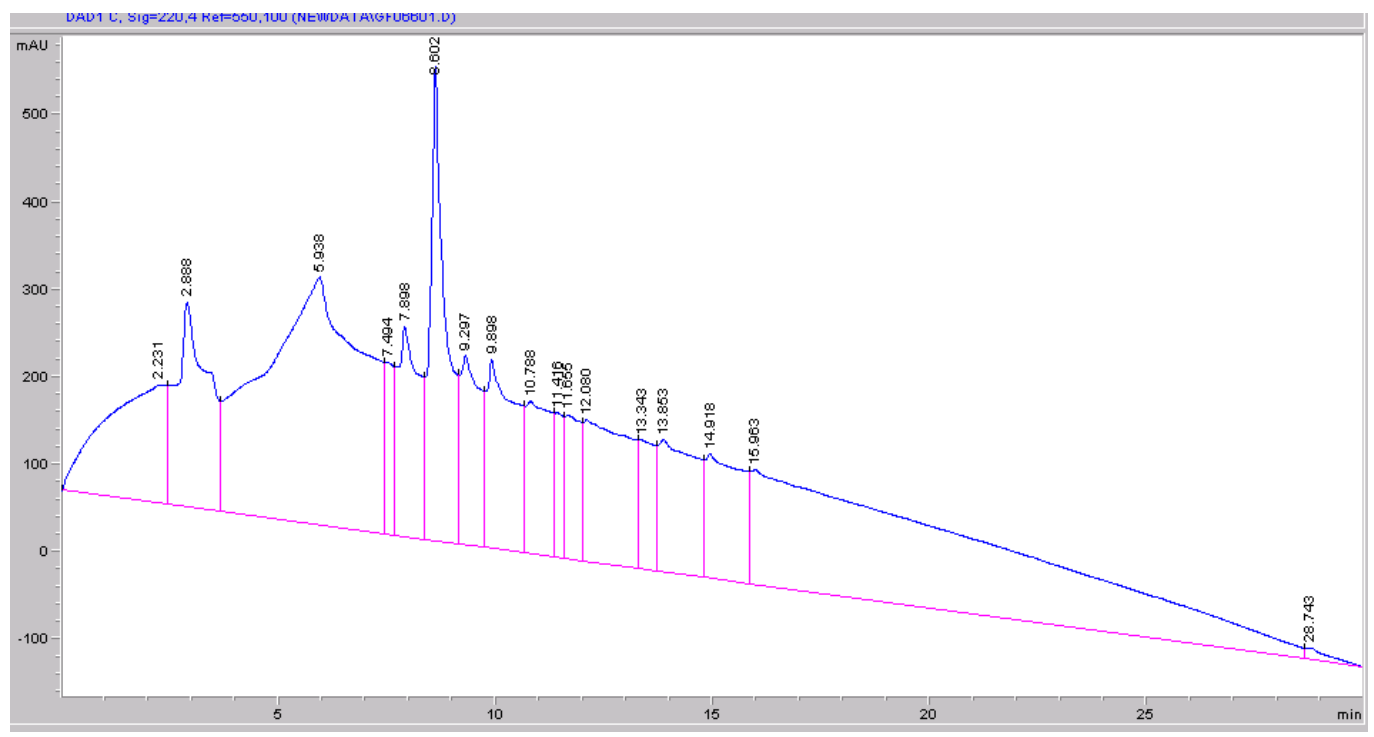

Supplemental Figure 8: HPLC chromatogram for hetero-oligomer 4 at $220 \mathrm{~nm}$ HPLC-MS: $\mathrm{t}_{\mathrm{R}}$ for 4, $8.6 \mathrm{~min}$; ES-LRMS calcd for $\mathrm{C}_{35} \mathrm{H}_{40} \mathrm{~N}_{9} \mathrm{O}_{11}\left(\mathrm{M}+\mathrm{H}^{+}\right) 762.3$, found 762.2 


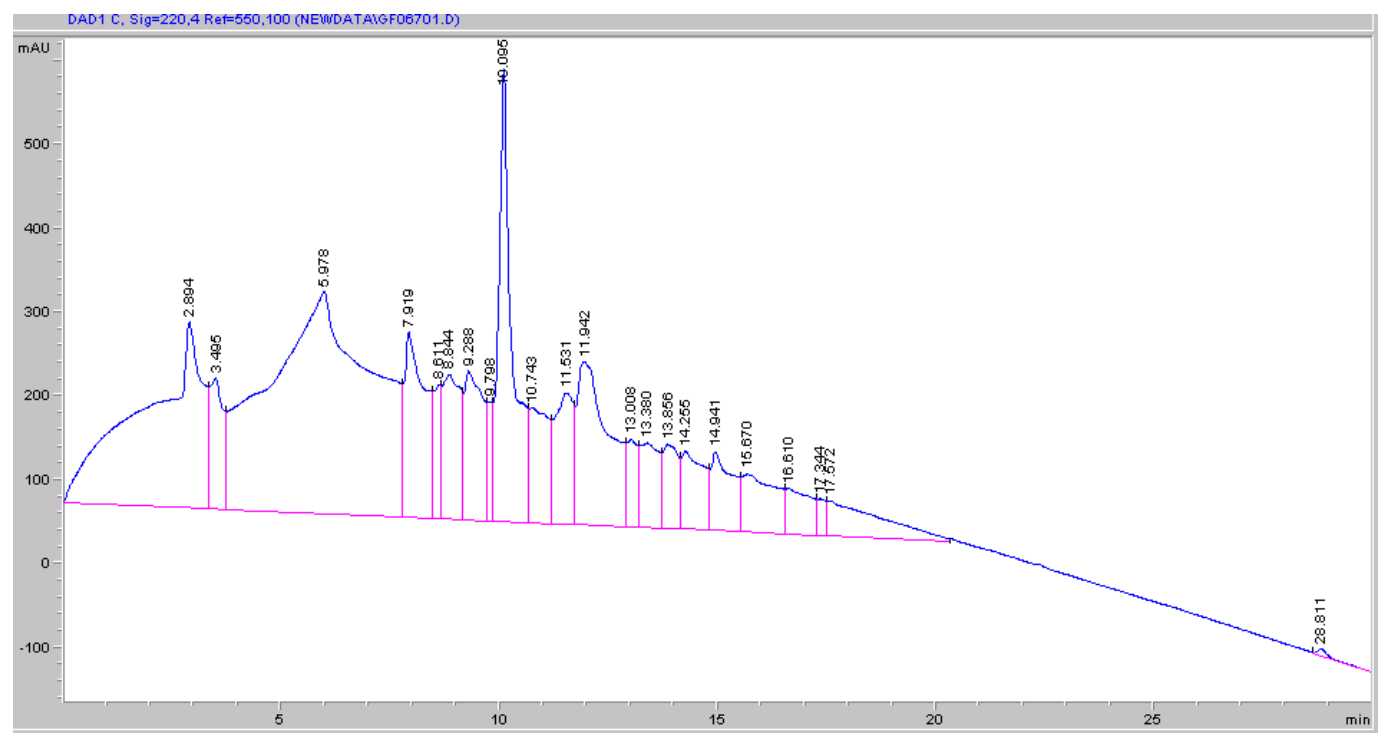

Supplemental Figure 9: HPLC chromatogram for hetero-oligomer 19 at $220 \mathrm{~nm}$ HPLC-MS: $\mathrm{t}_{\mathrm{R}}$ for 19, $10.1 \mathrm{~min}$; ES-LRMS calcd for $\mathrm{C}_{35} \mathrm{H}_{40} \mathrm{~N}_{9} \mathrm{O}_{11}\left(\mathrm{M}+\mathrm{H}^{+}\right)$762.3, found 762.2

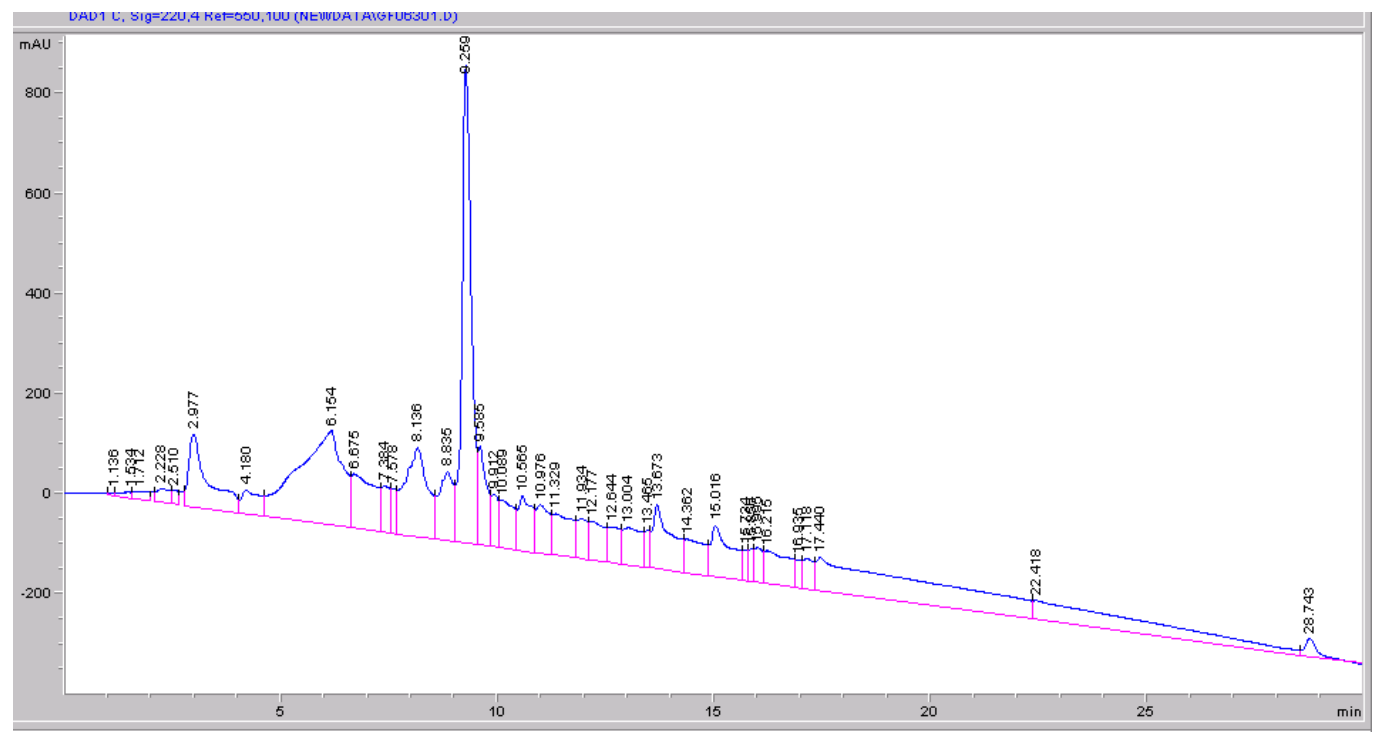

Supplemental Figure 10: HPLC chromatogram for hetero-oligomer 20 at $220 \mathrm{~nm}$ HPLC-MS: $t_{R}$ for 20, 9.3 min; ES-LRMS calcd for $\mathrm{C}_{35} \mathrm{H}_{40} \mathrm{~N}_{9} \mathrm{O}_{11}\left(\mathrm{M}+\mathrm{H}^{+}\right) 762.3$, found 762.2 


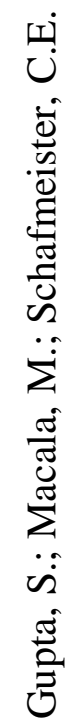

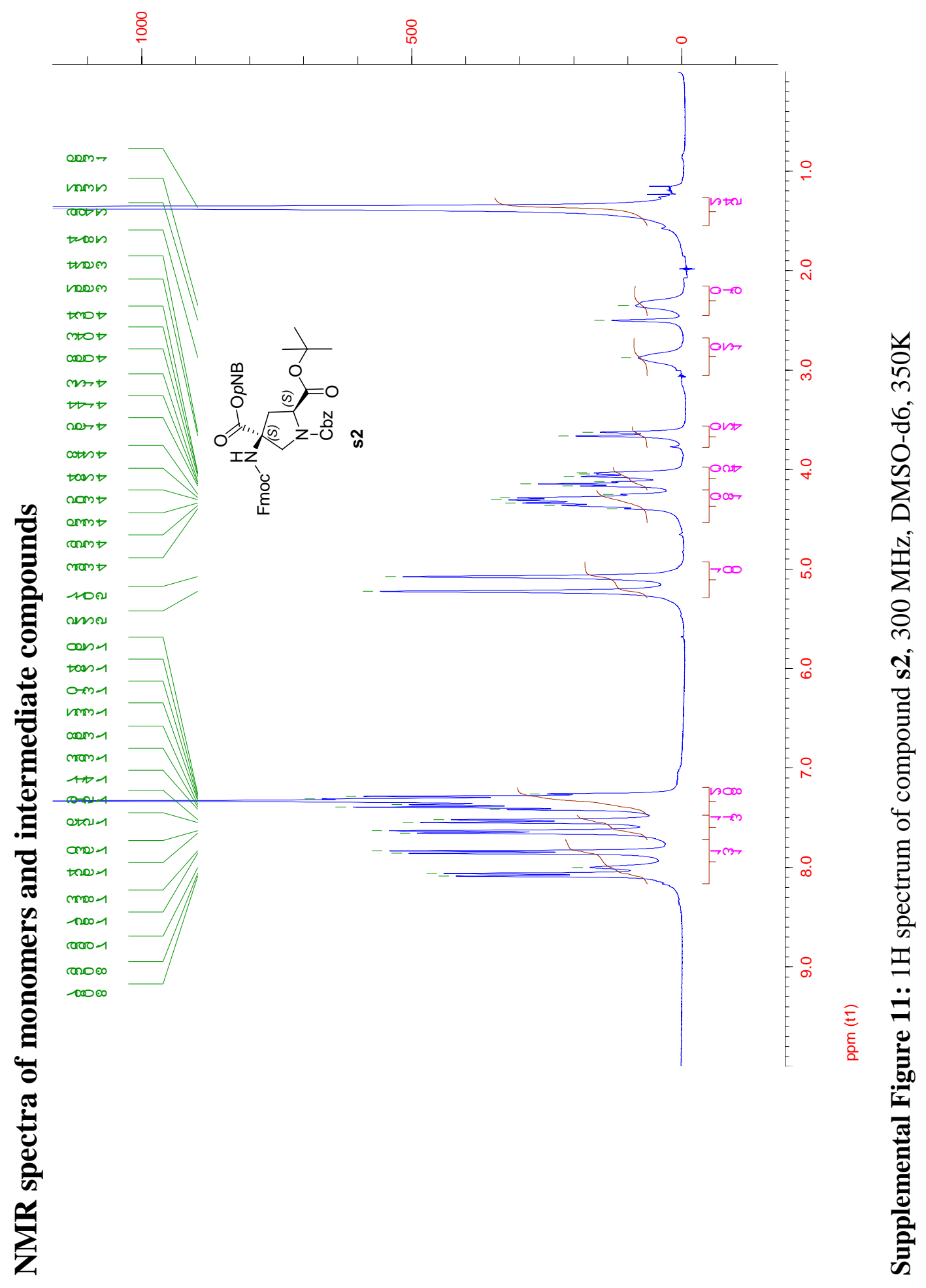


U
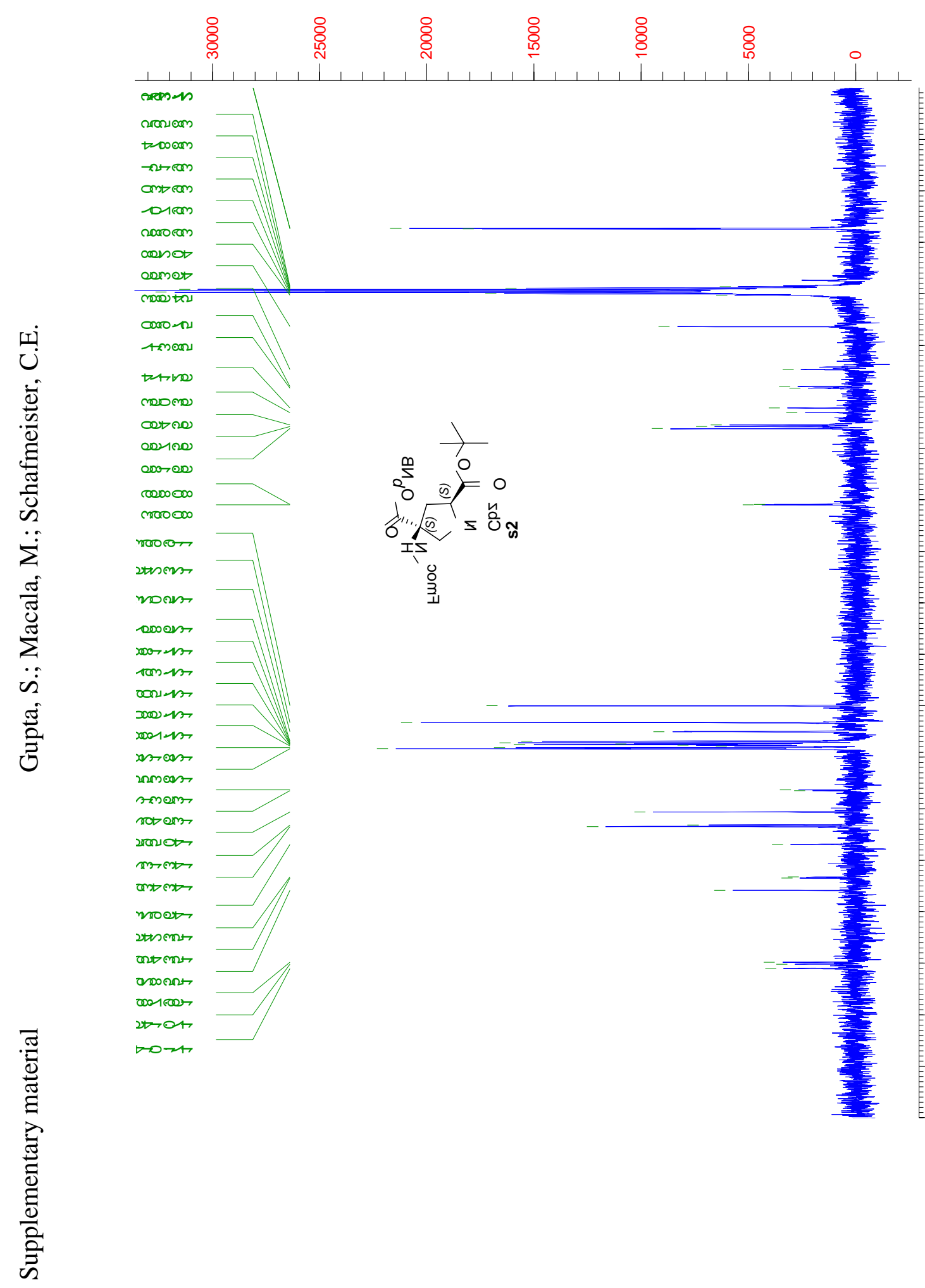

हี 


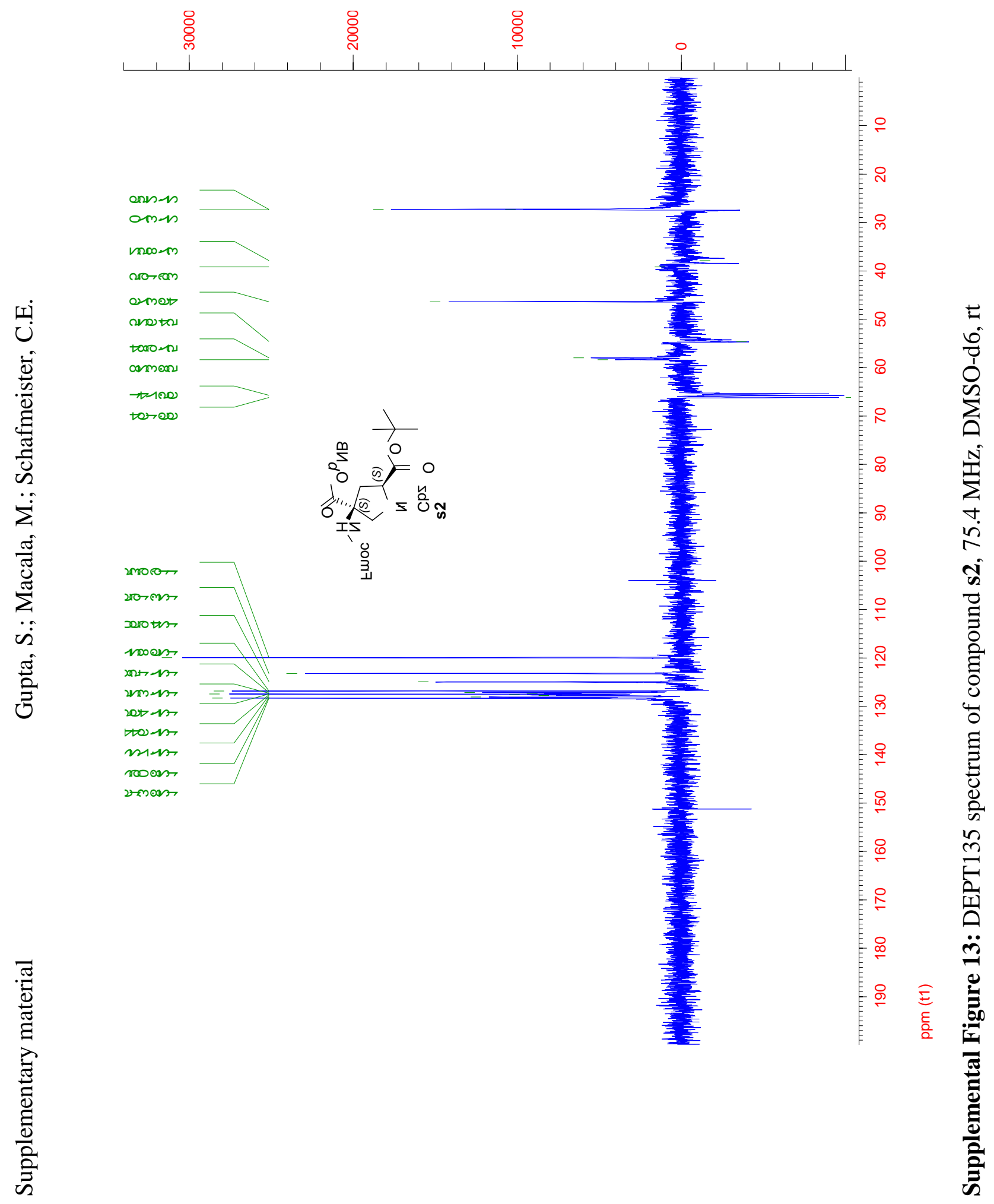




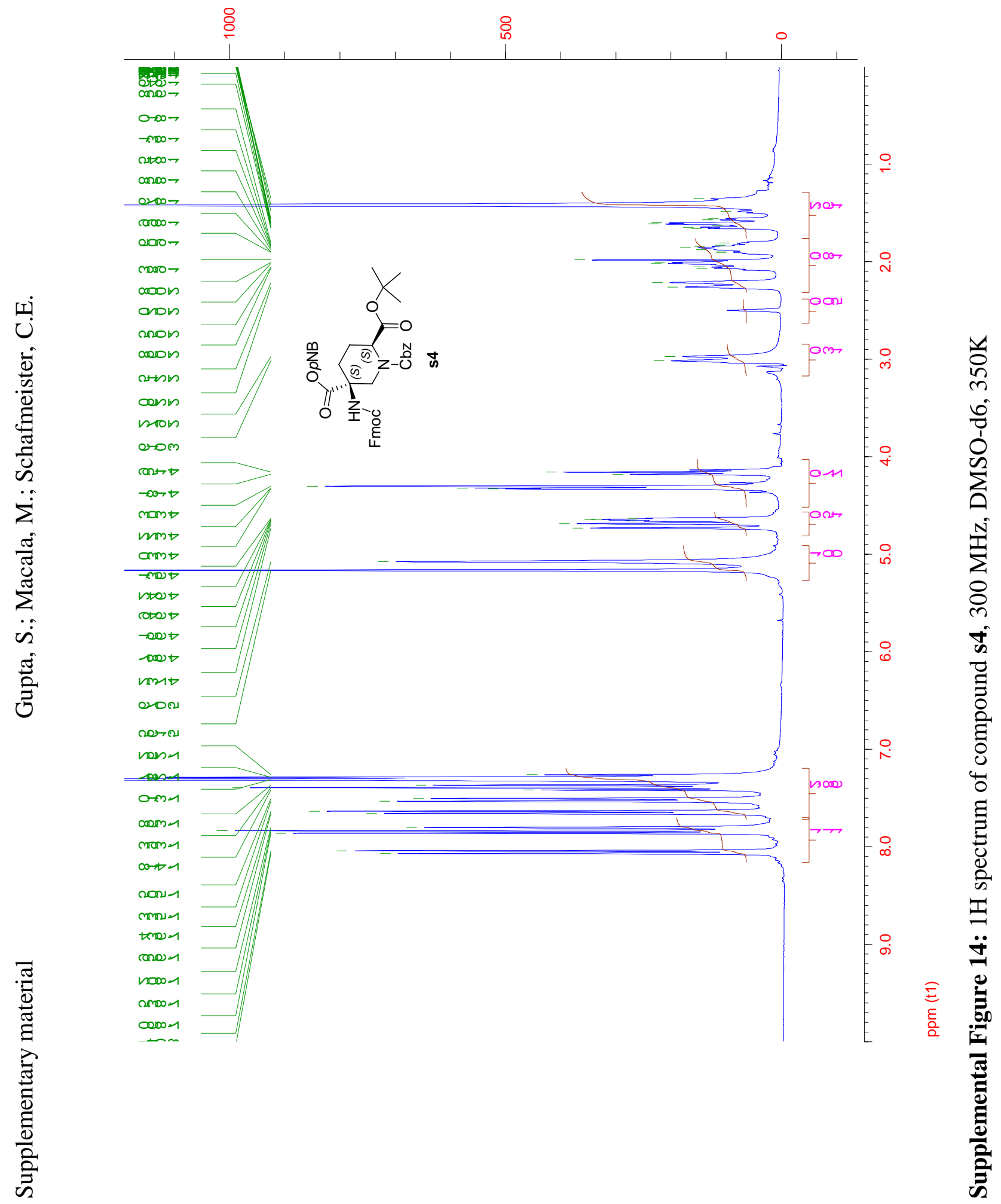




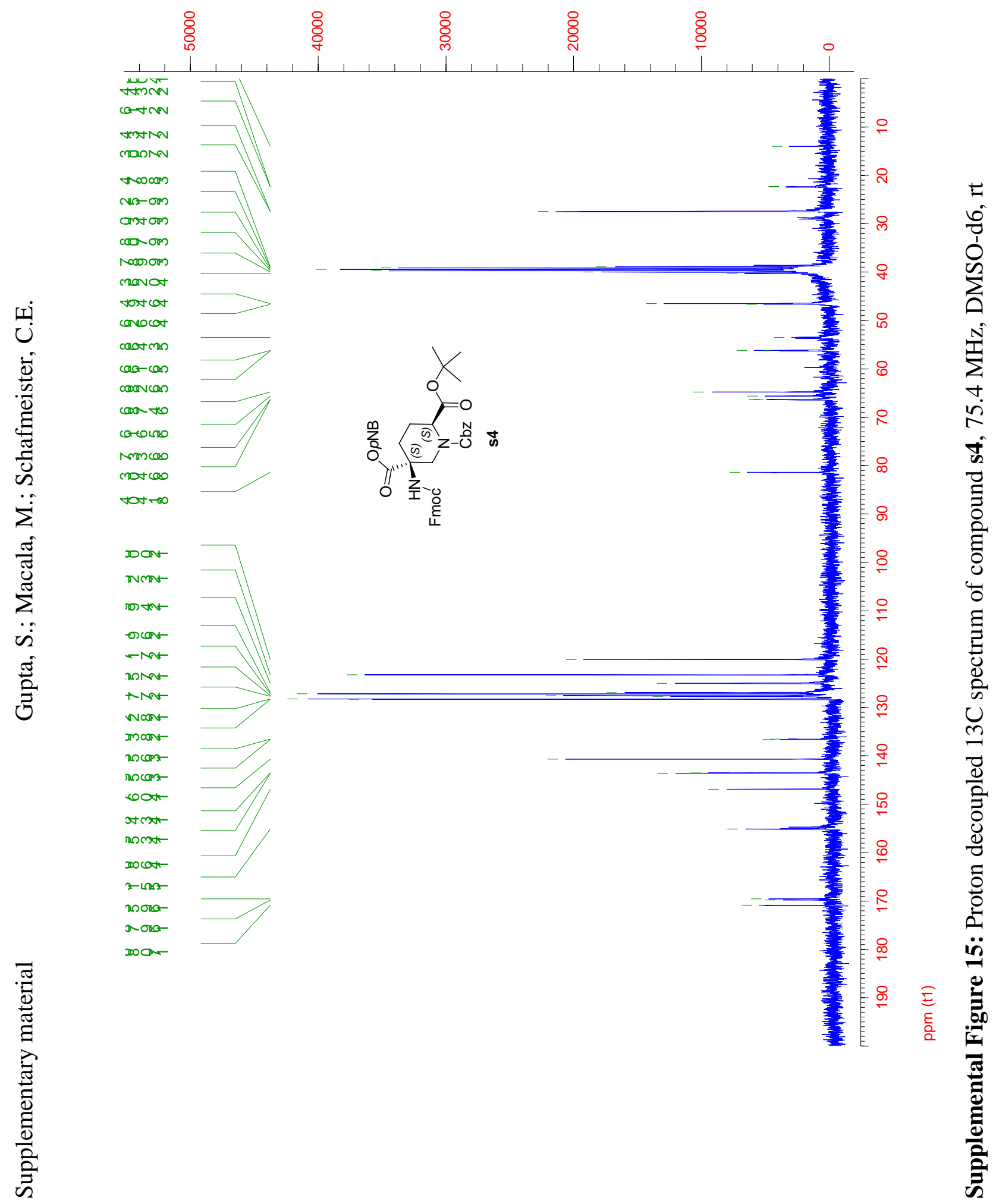




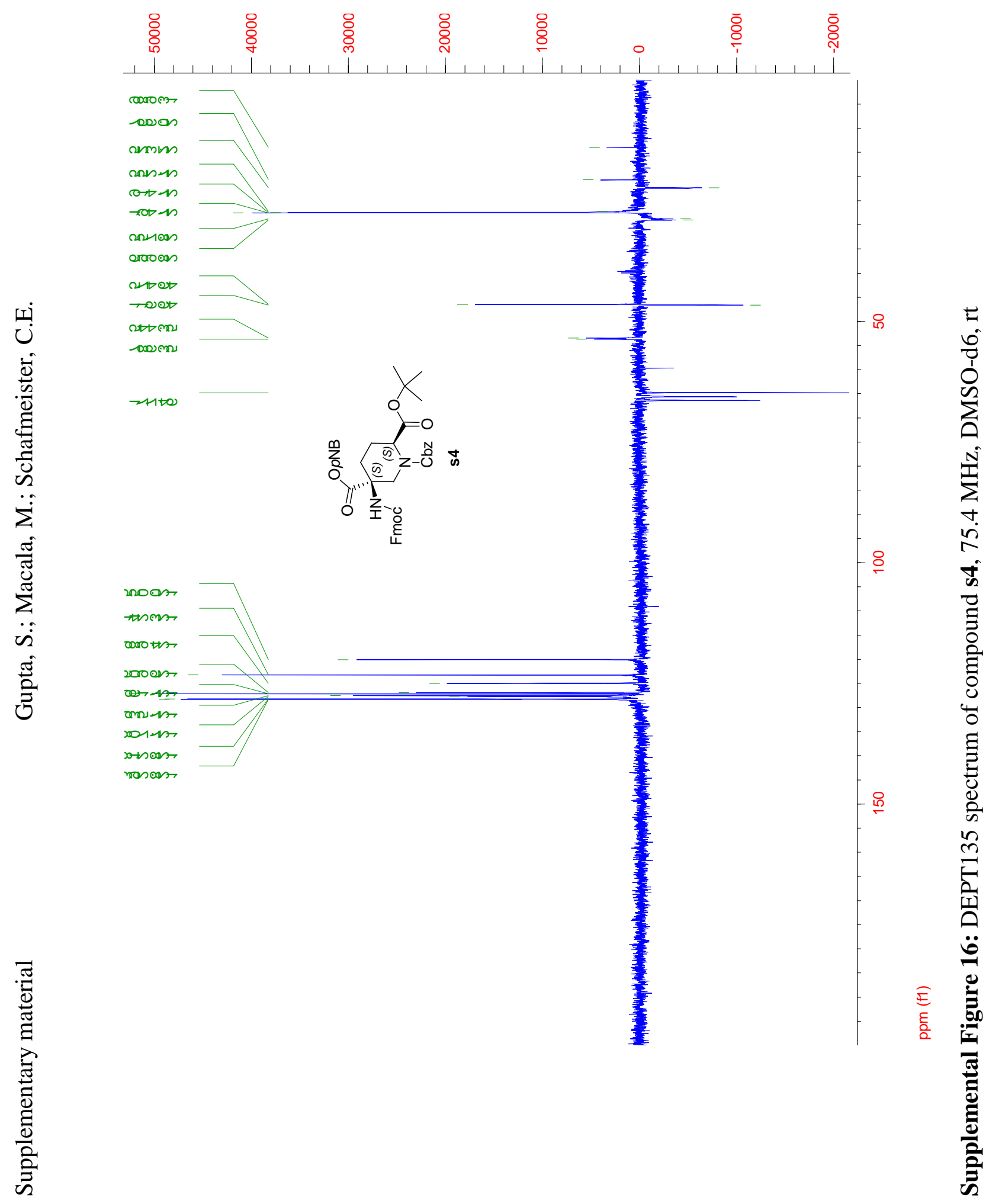




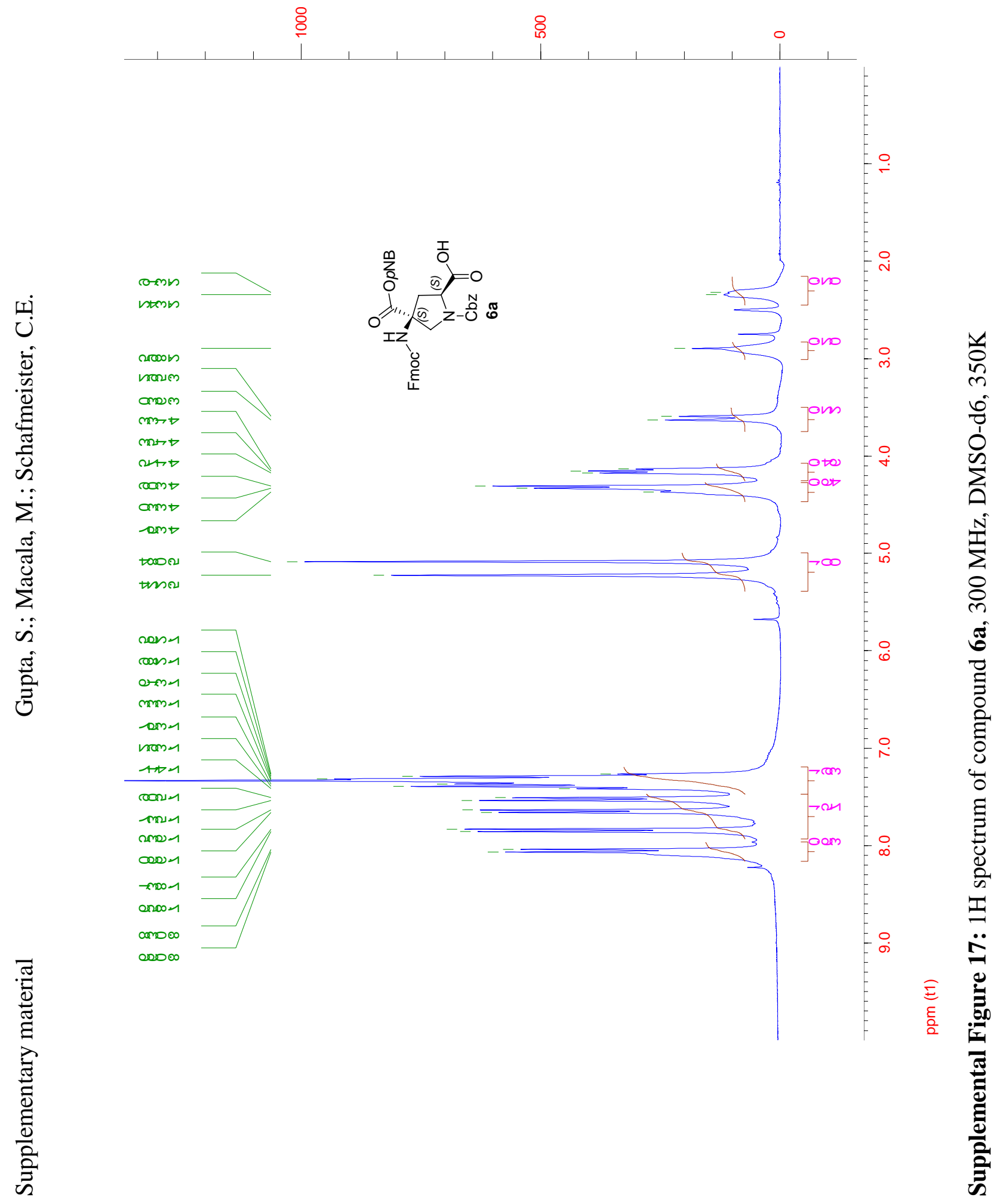




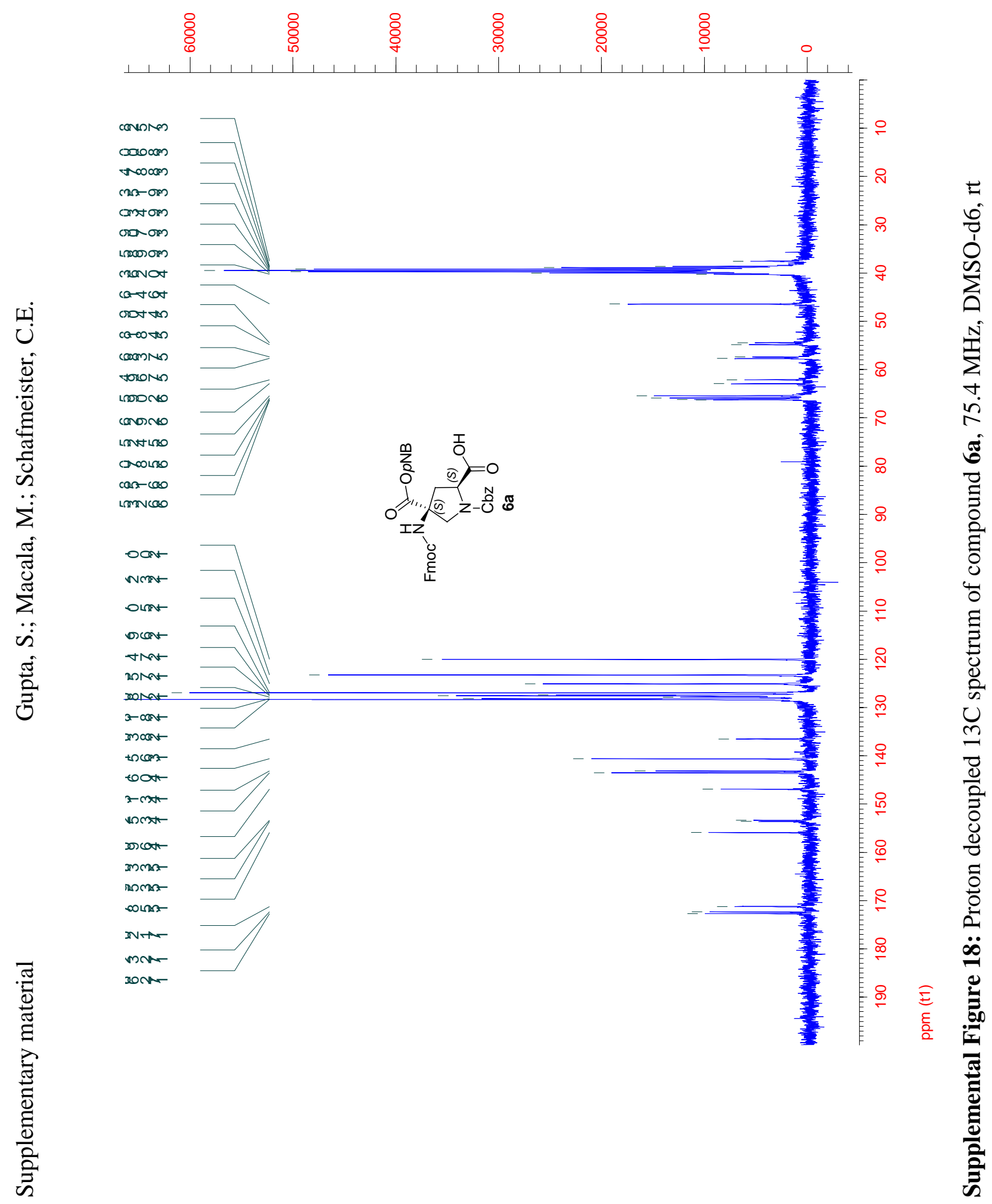




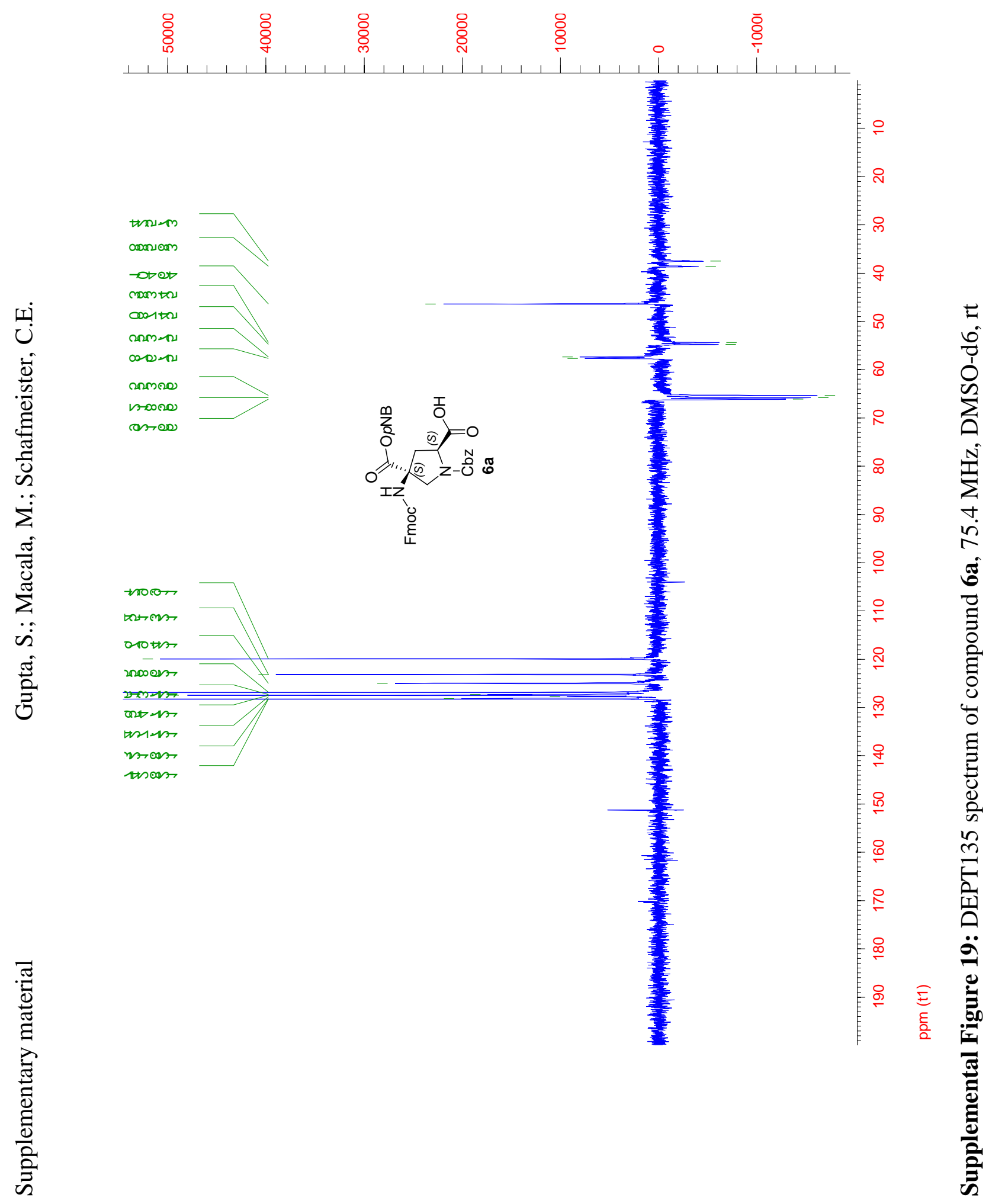




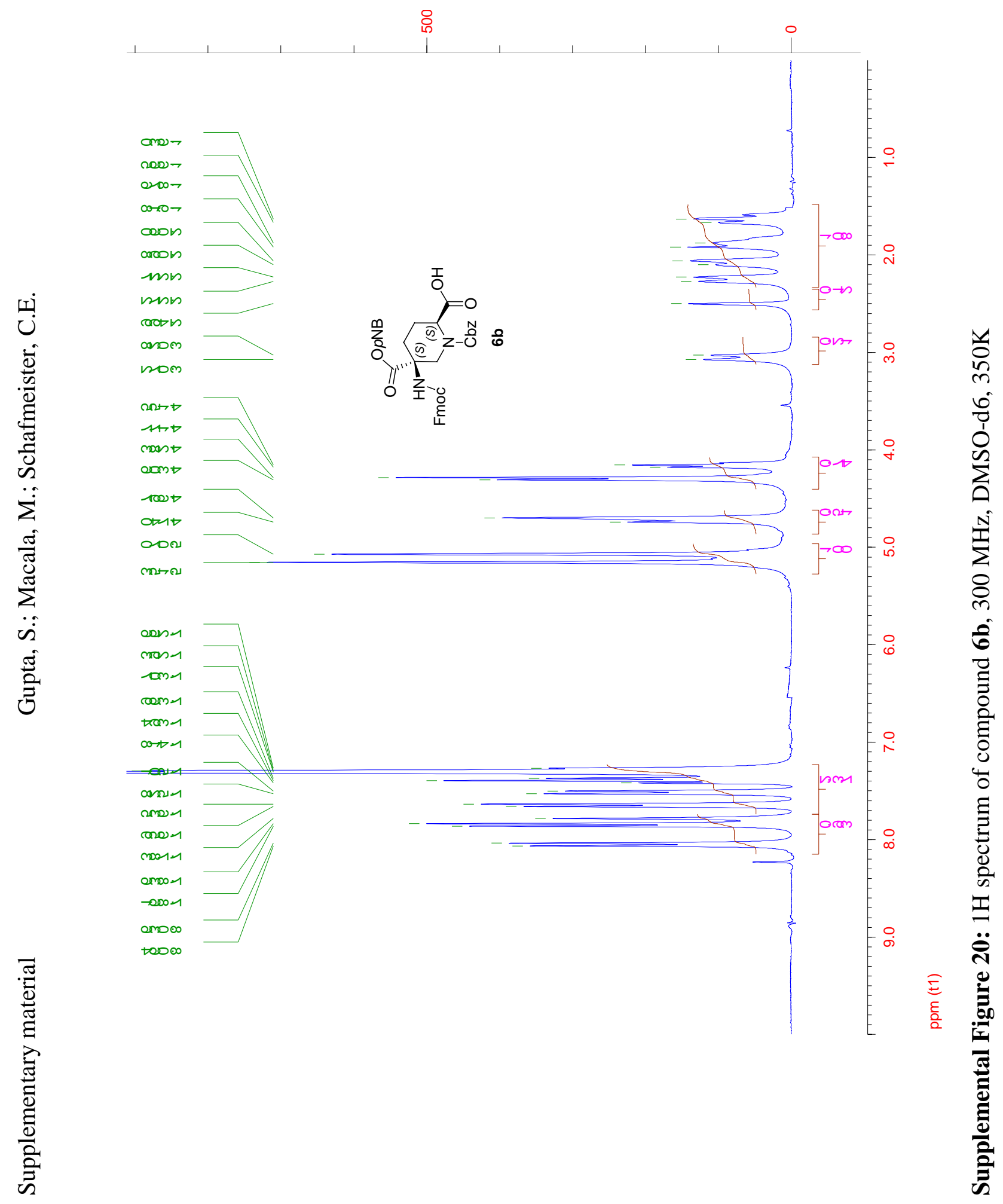




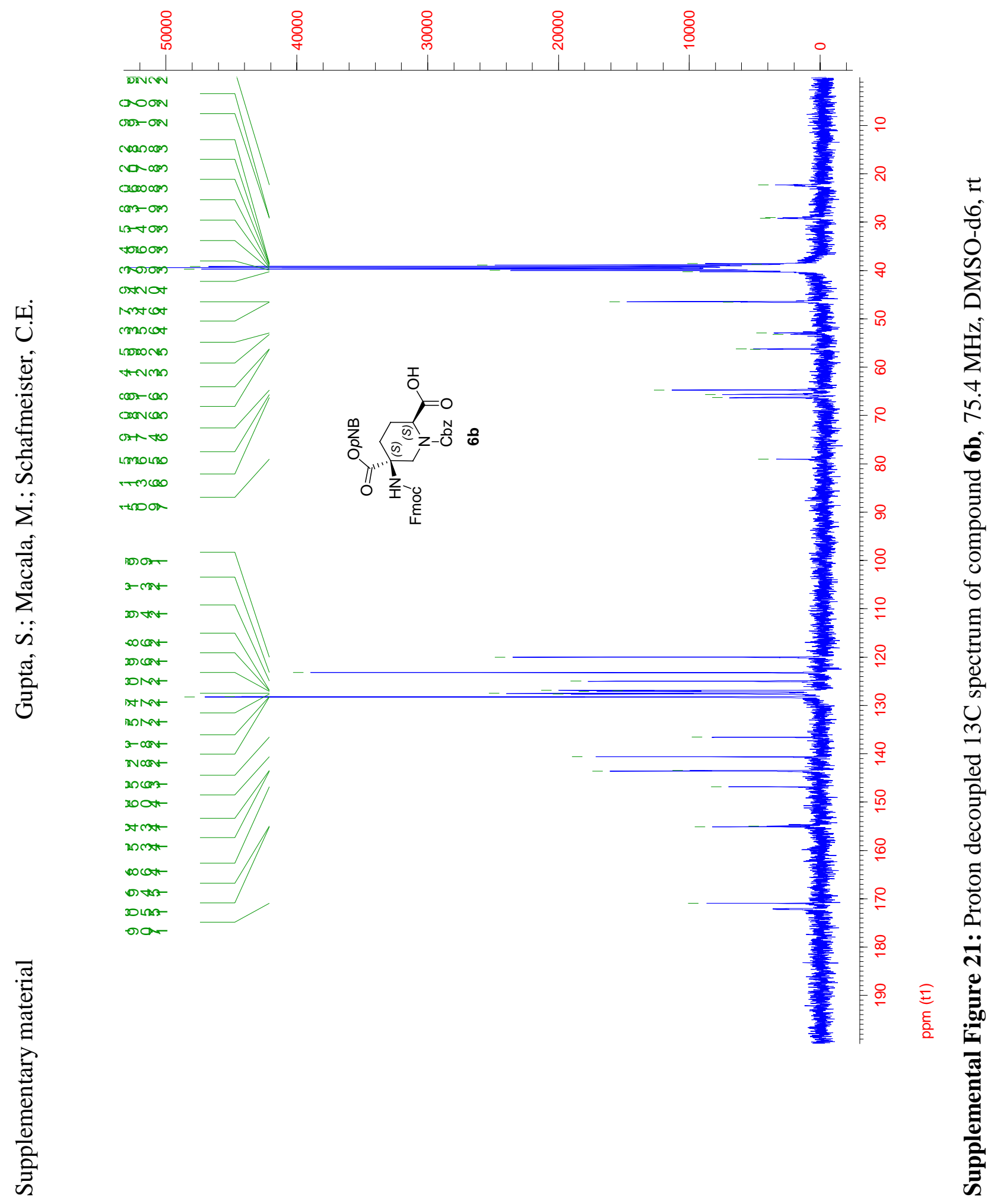




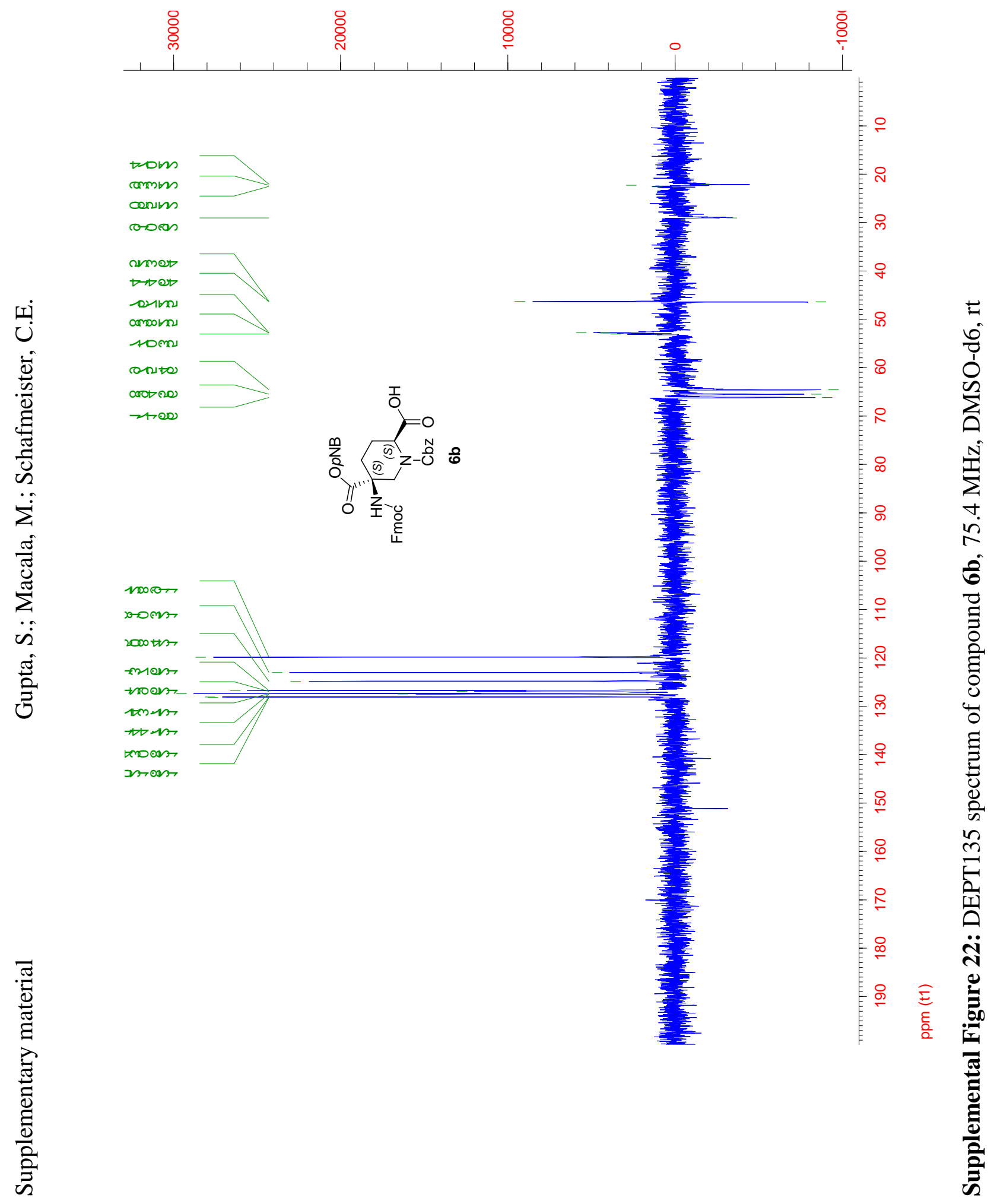




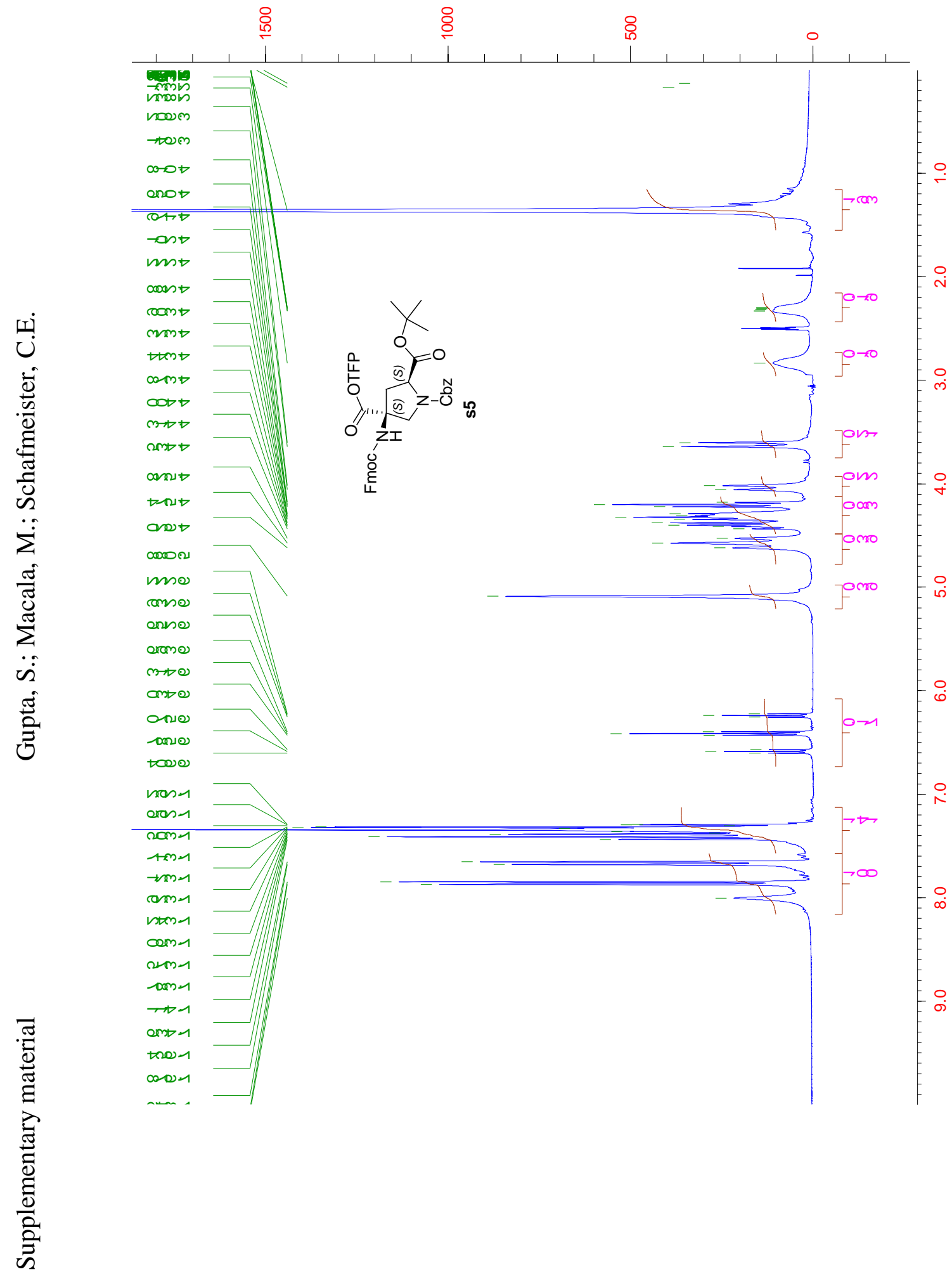




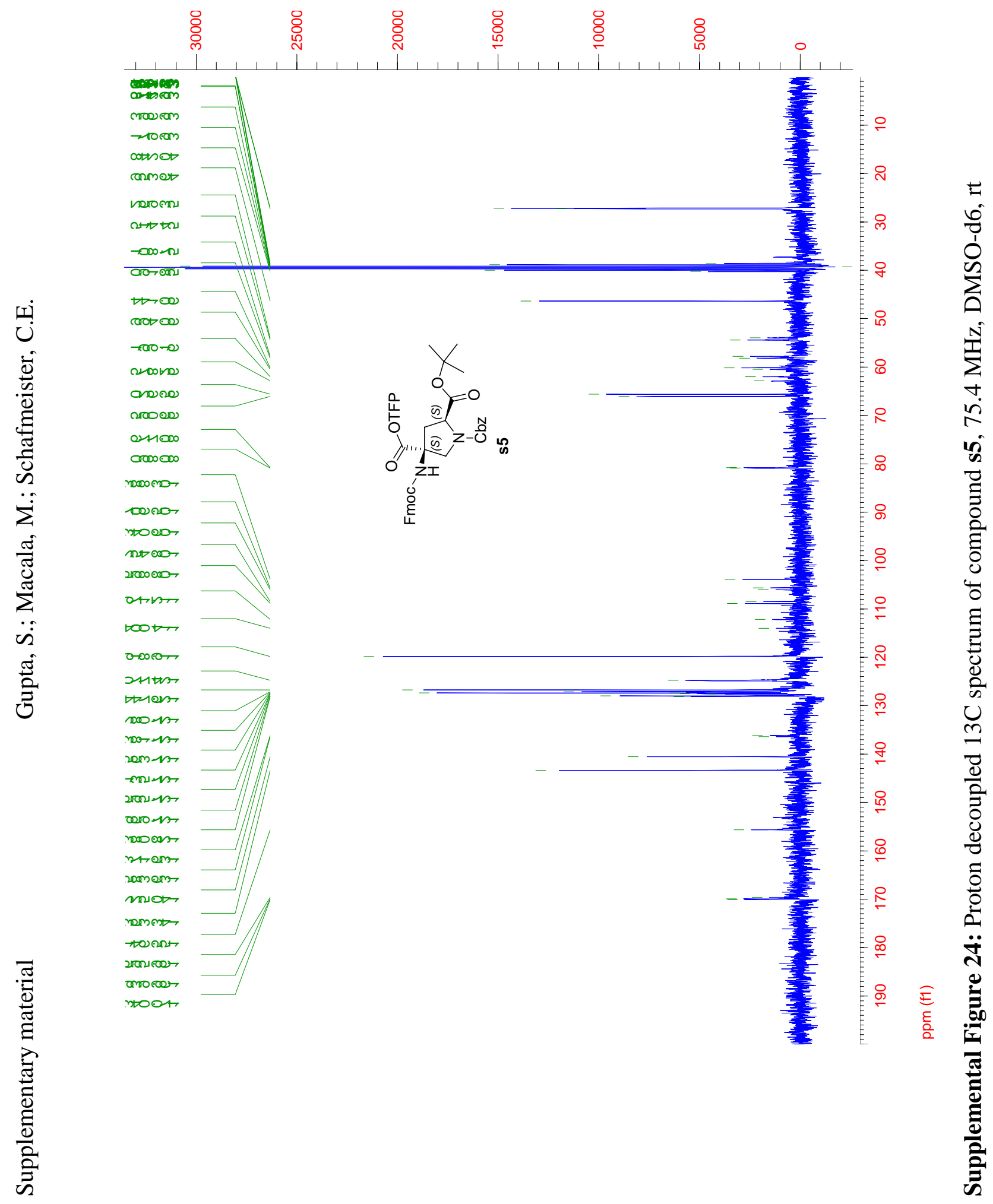




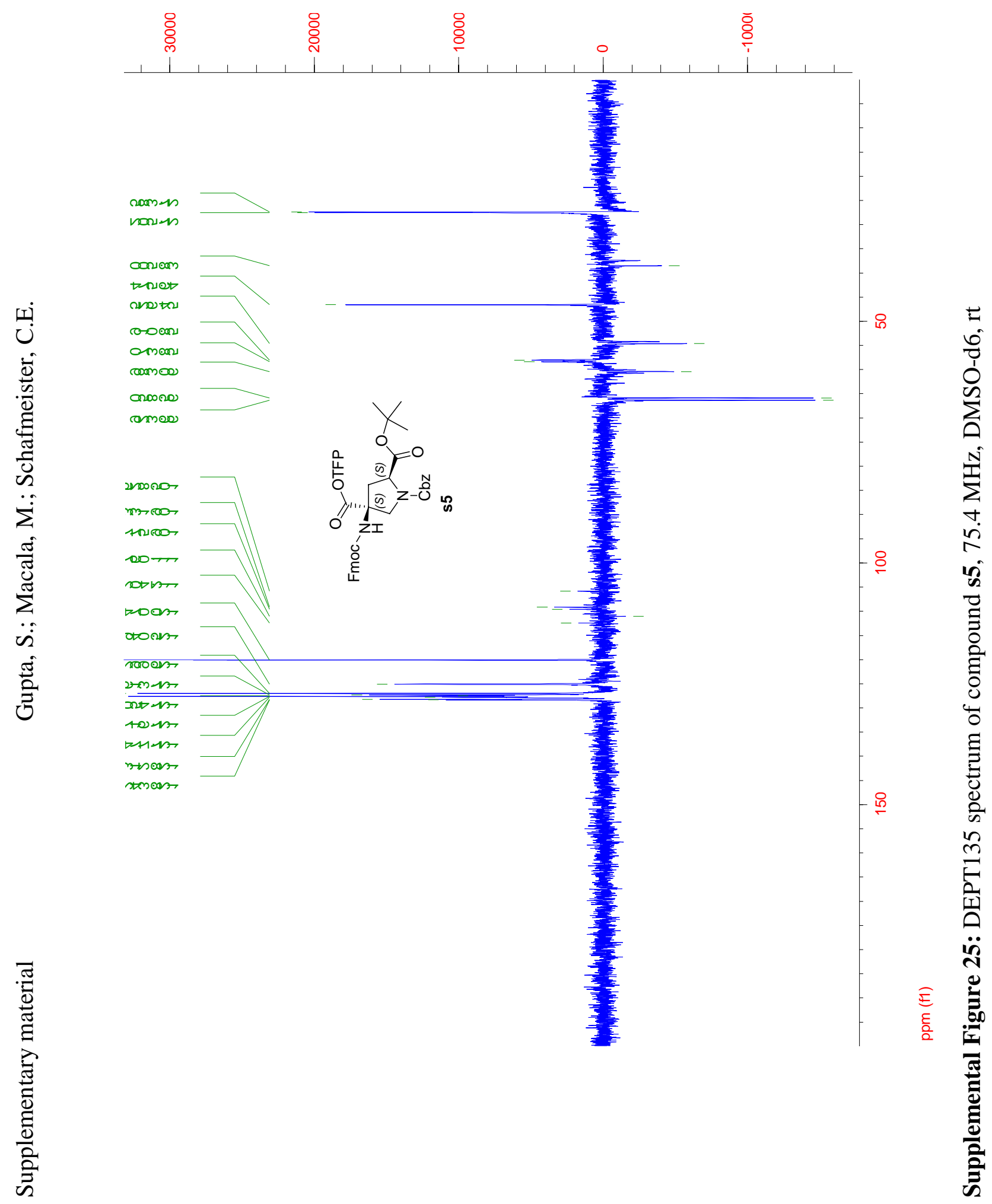




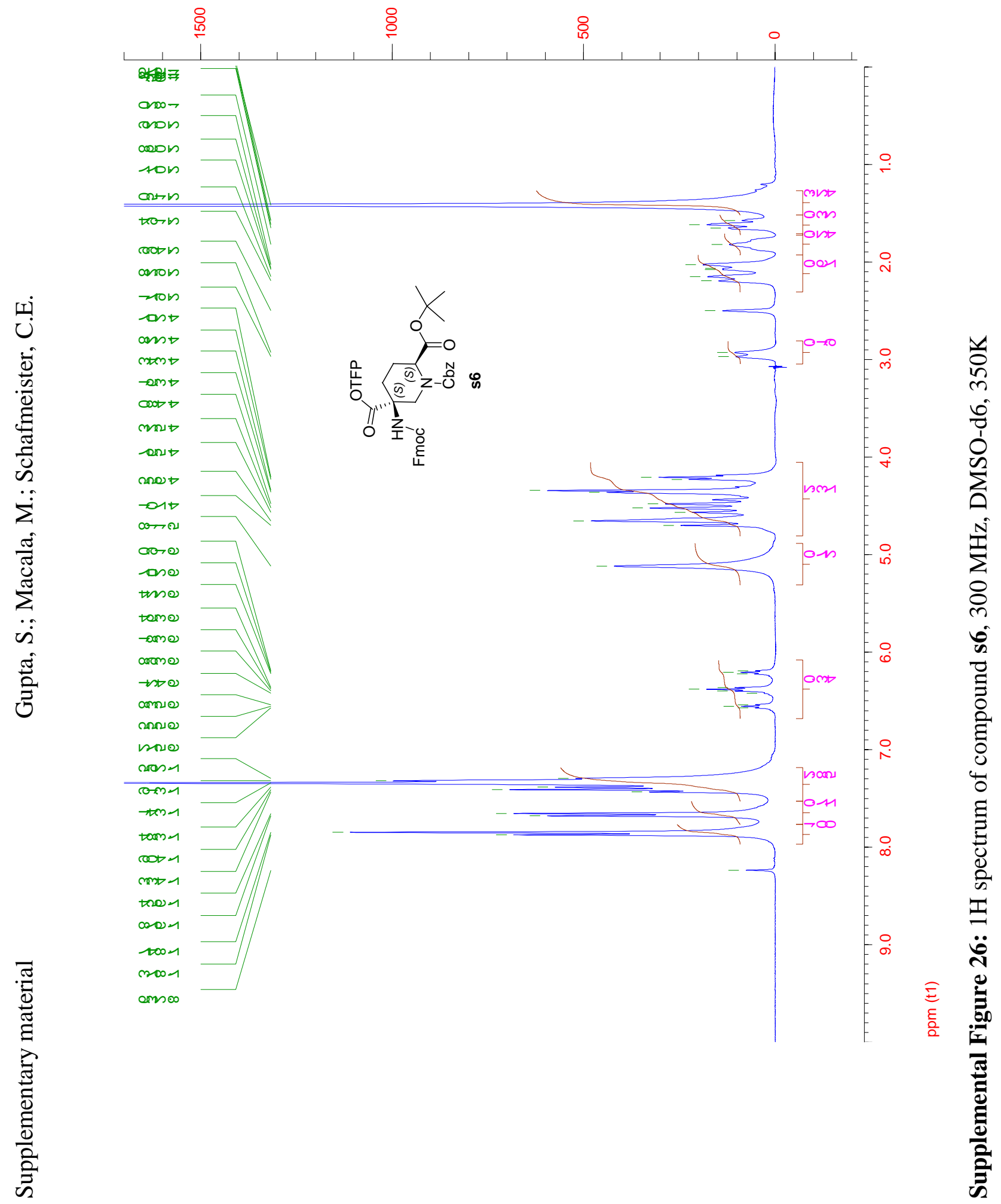




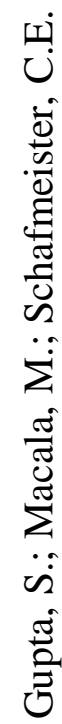
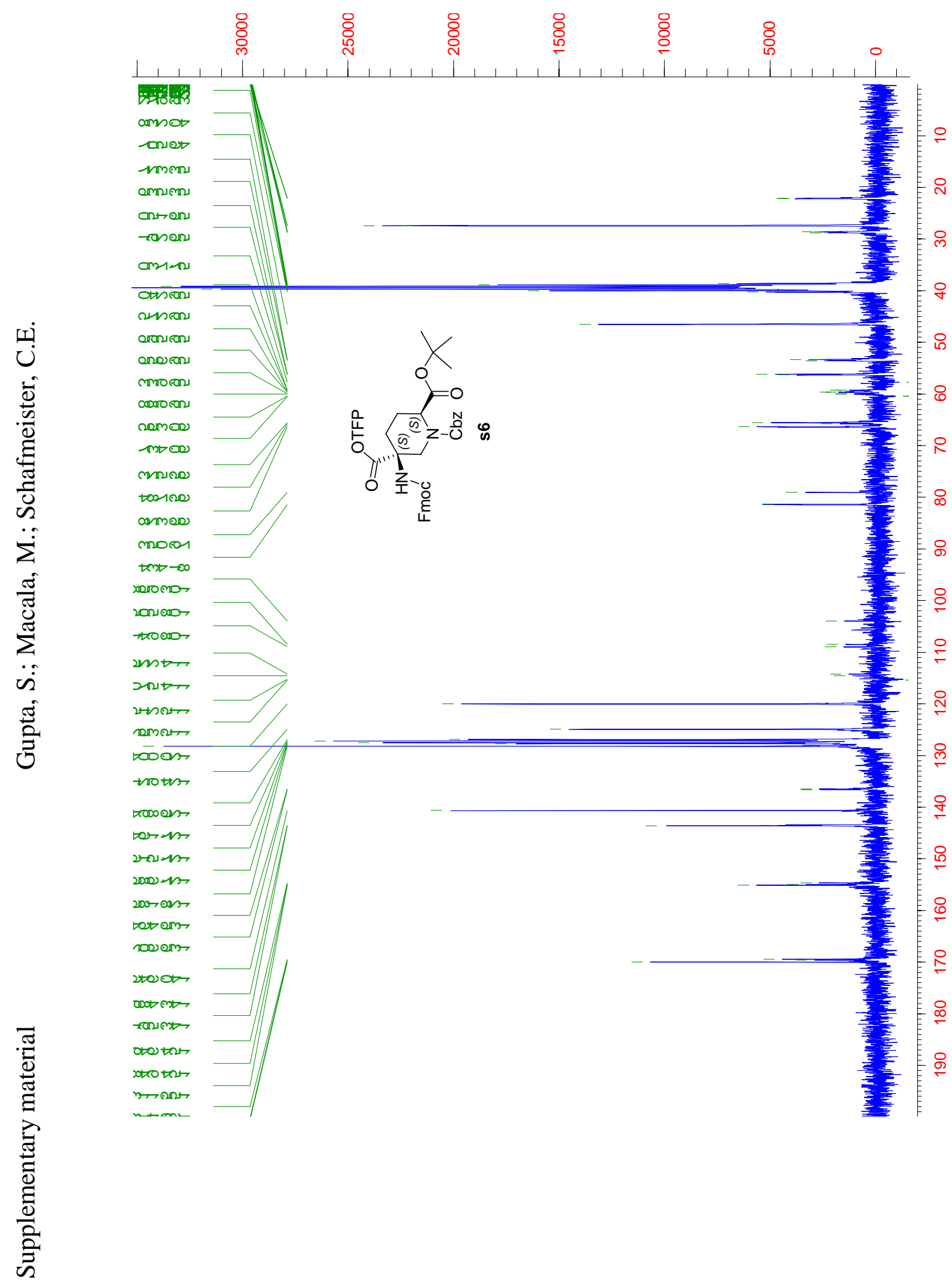

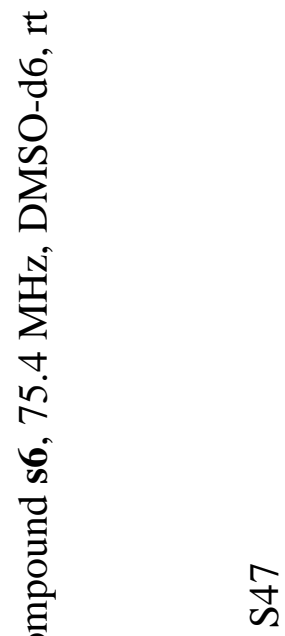




$$
\|
$$




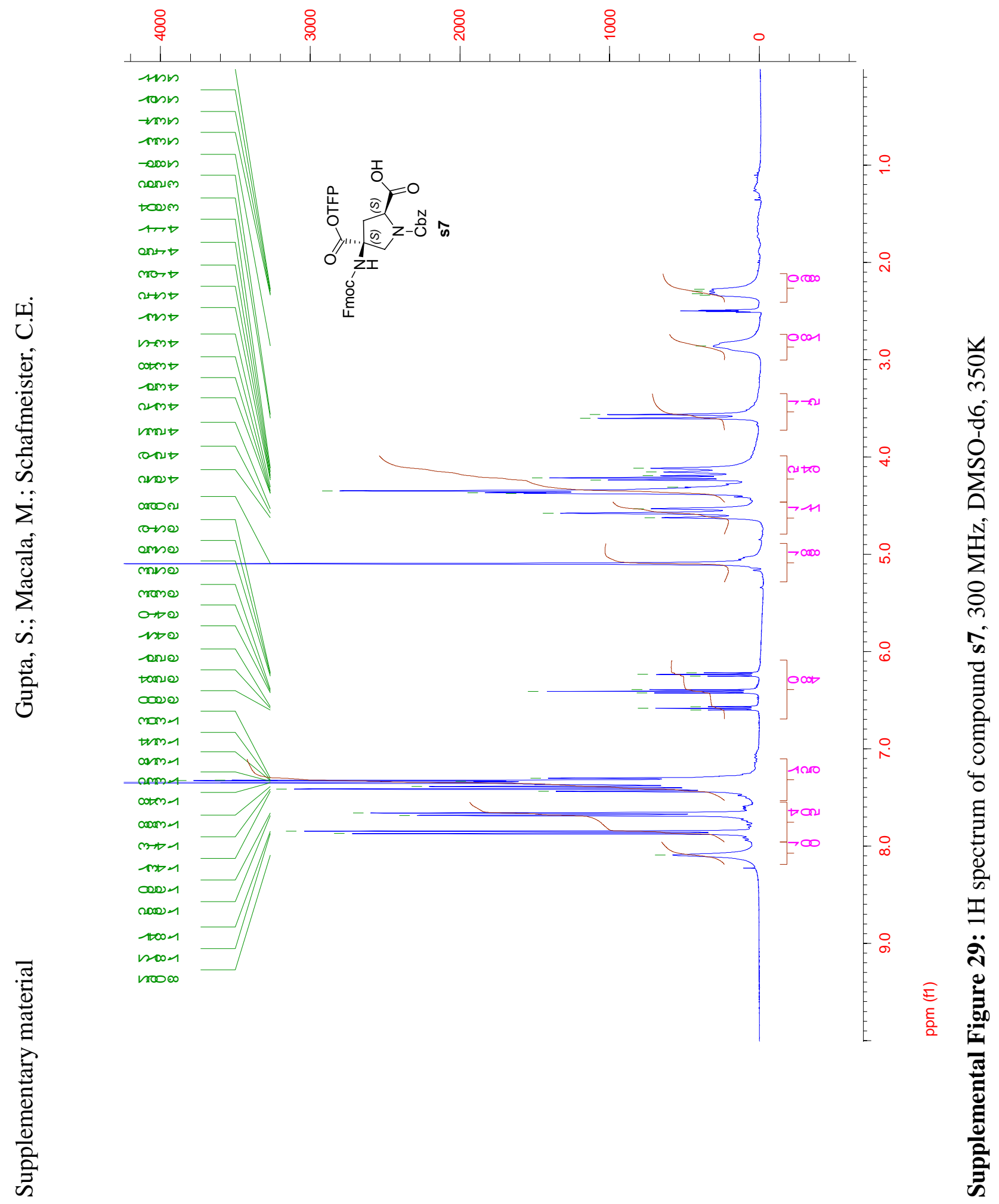




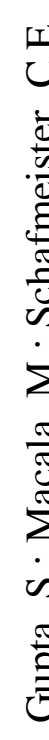

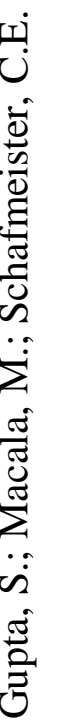

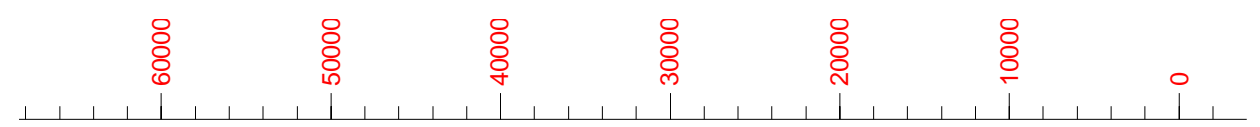

\section{ths}

-ostan

antran

क्वान्क

orotan

sonos

castors

the tre

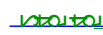

coses

क्या-

वसान्य

आவ

rotes

बero-0

ल्भ⿰亻⿻上丨冂口

लมฉ०

tore

-ootor

xnow

namer

tasster

क्या

mot

ग050

sootes

tooes

xastr.

ob

tount

toons

vercos

2 mors

अथक

want

atser

अमका

काला

क्यलि

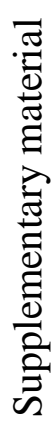
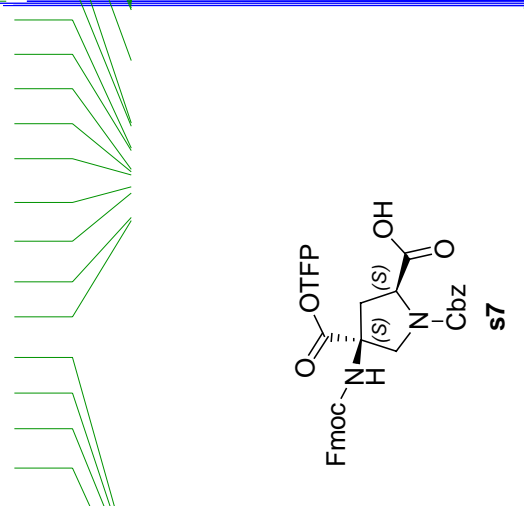

ansat

conost

somest 


$$
\|+1
$$




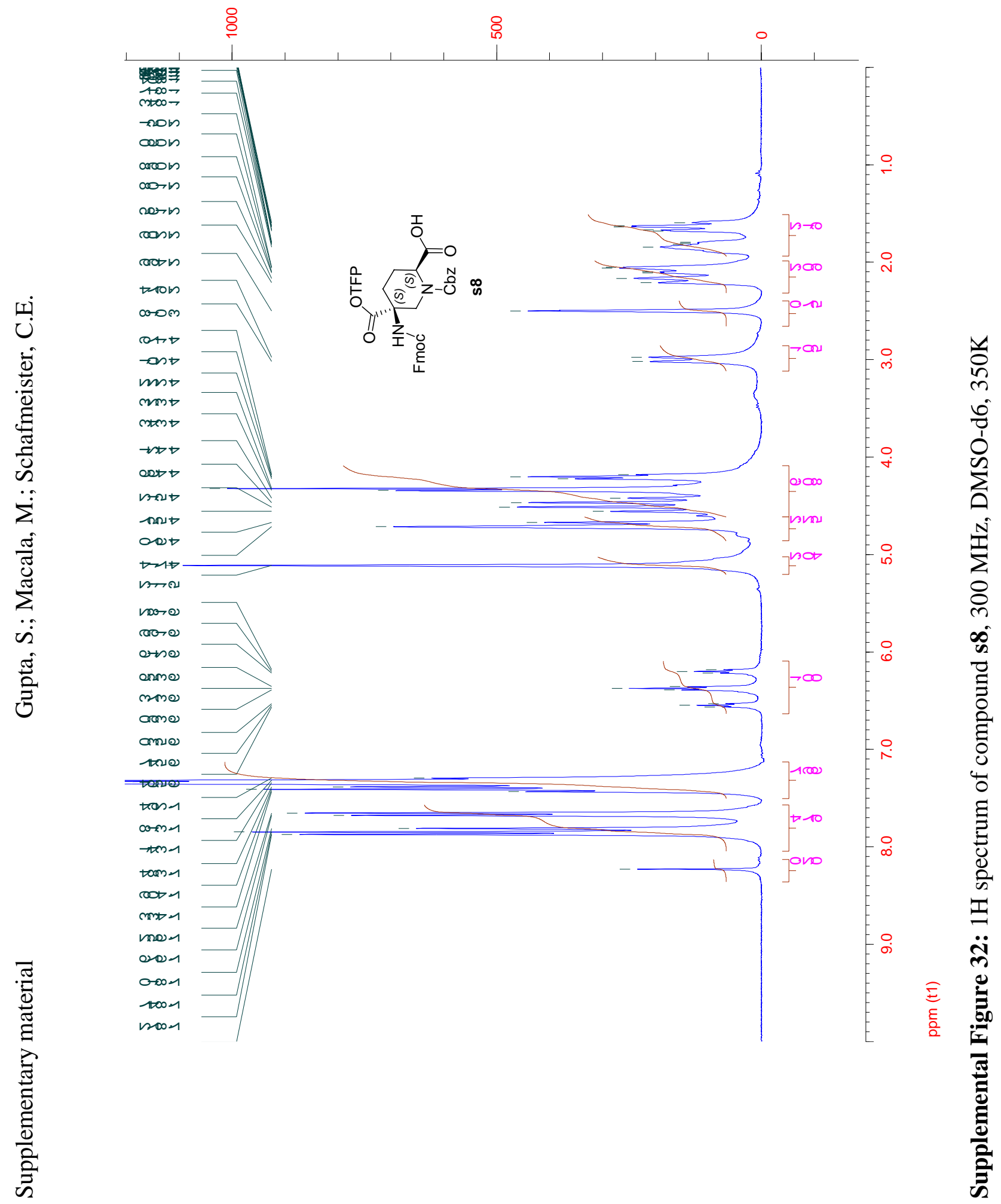




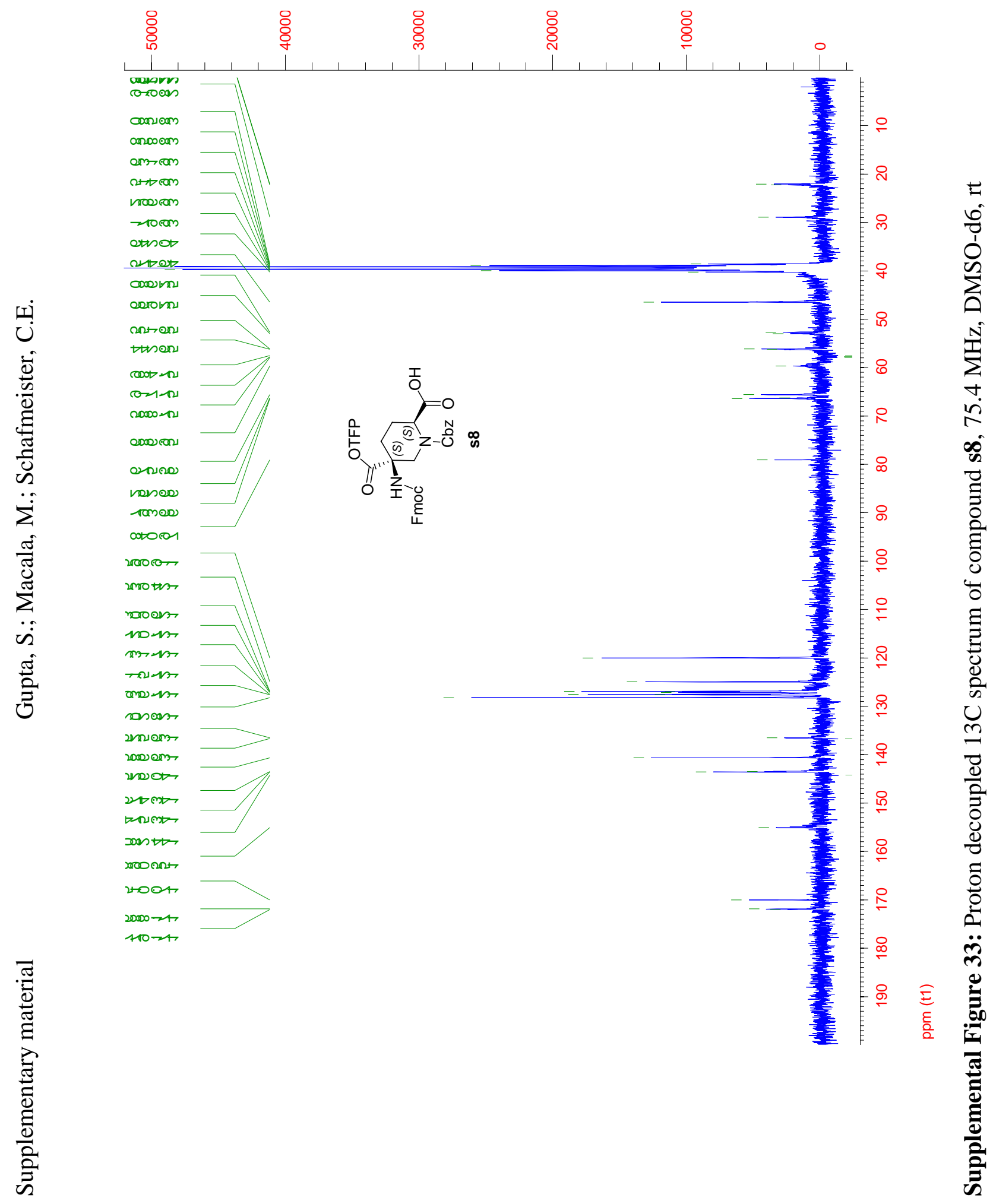




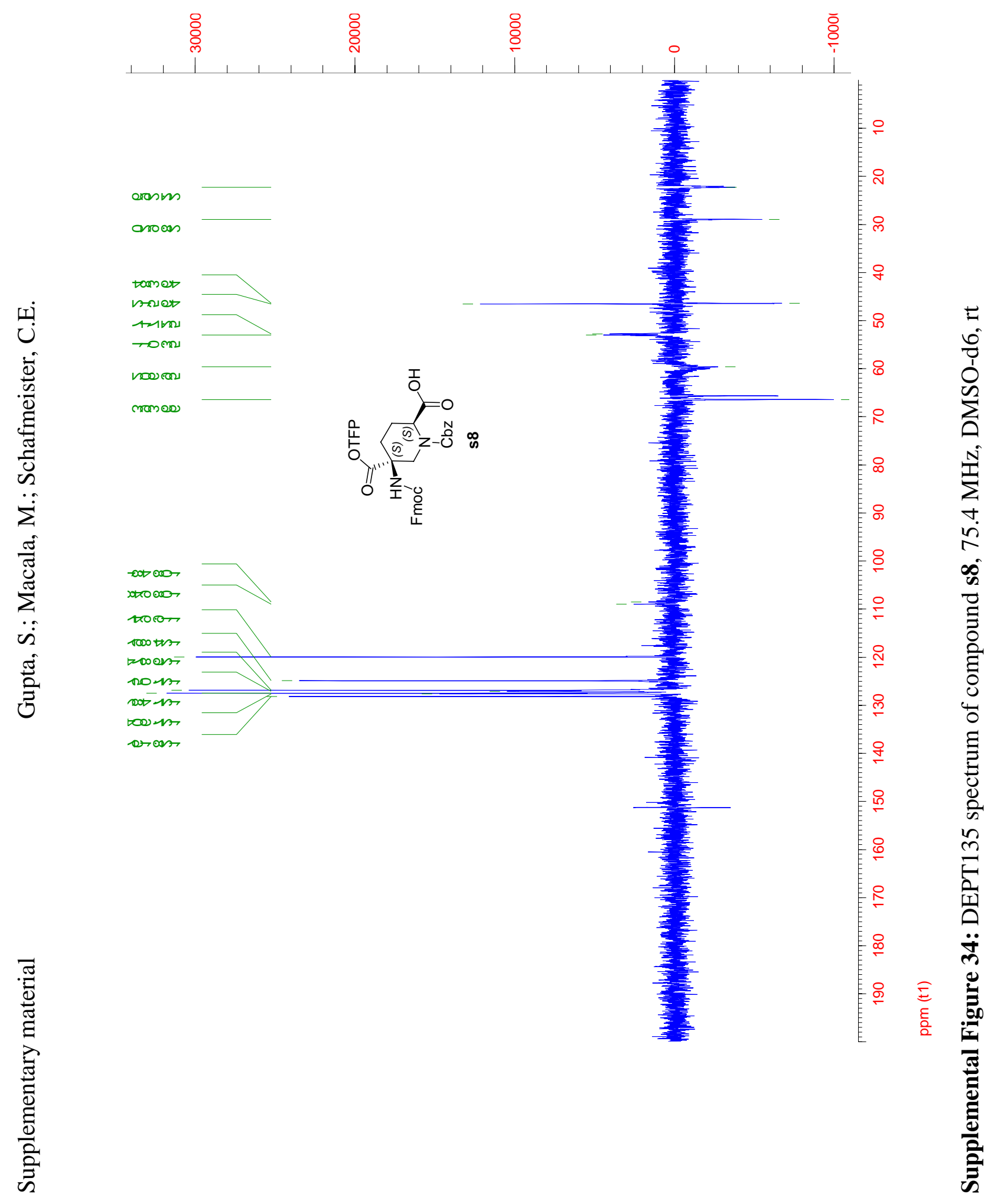




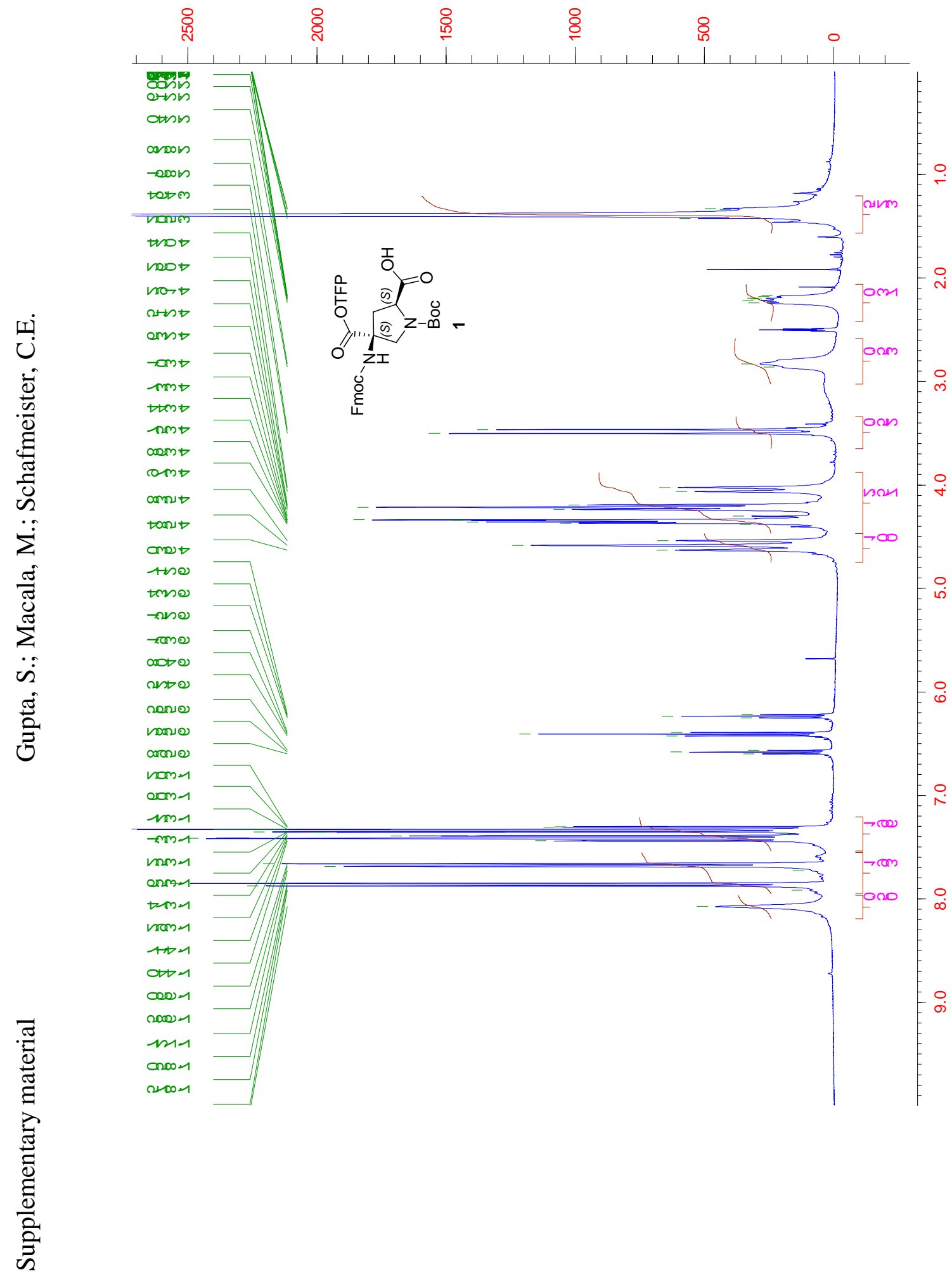

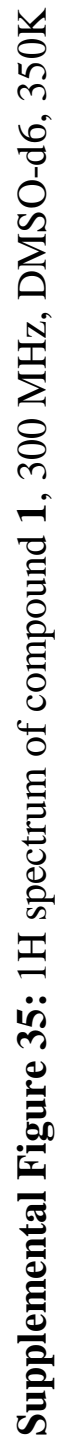




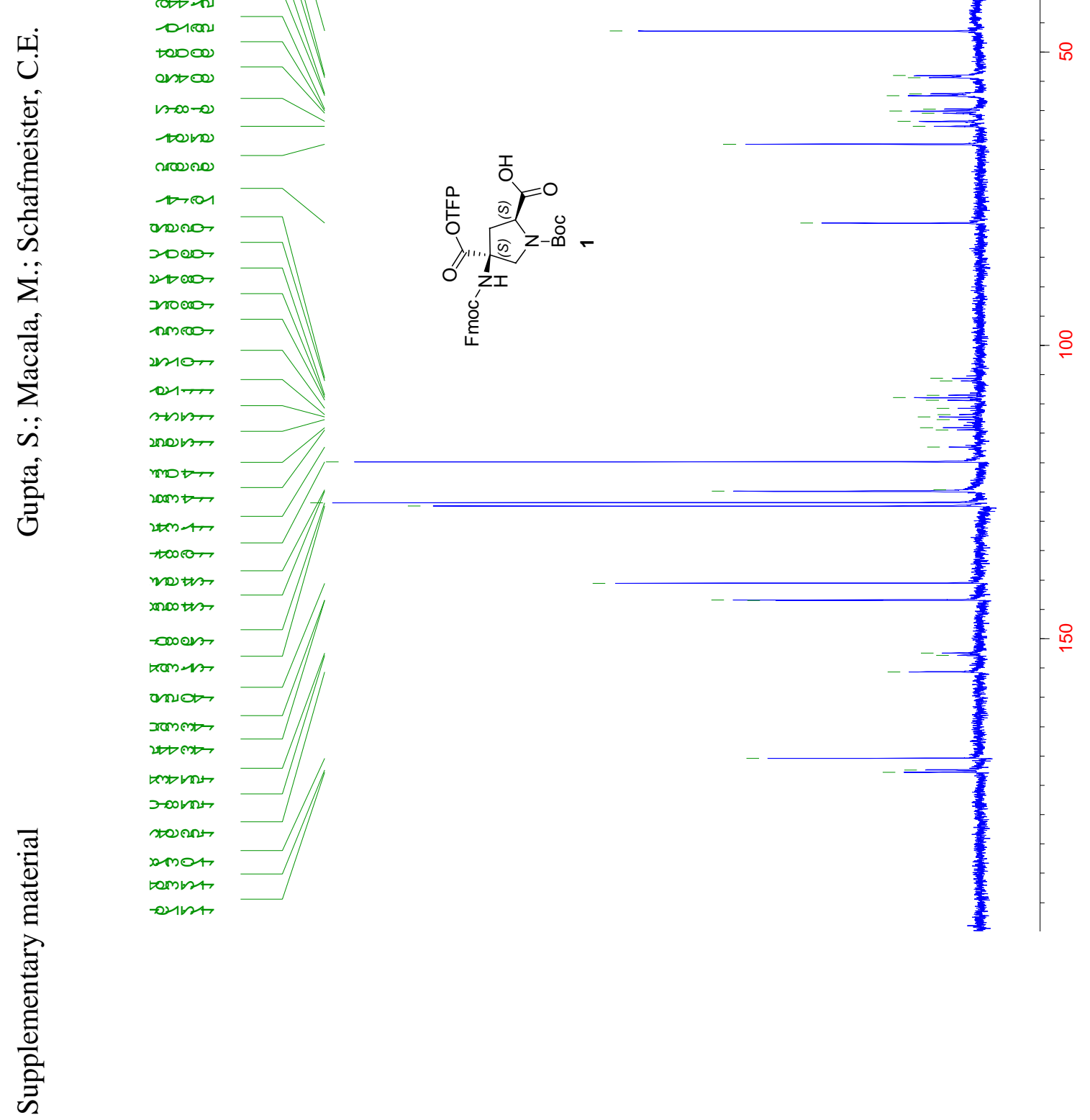

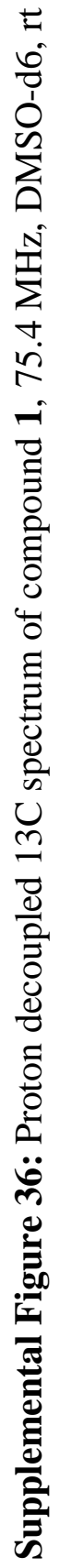




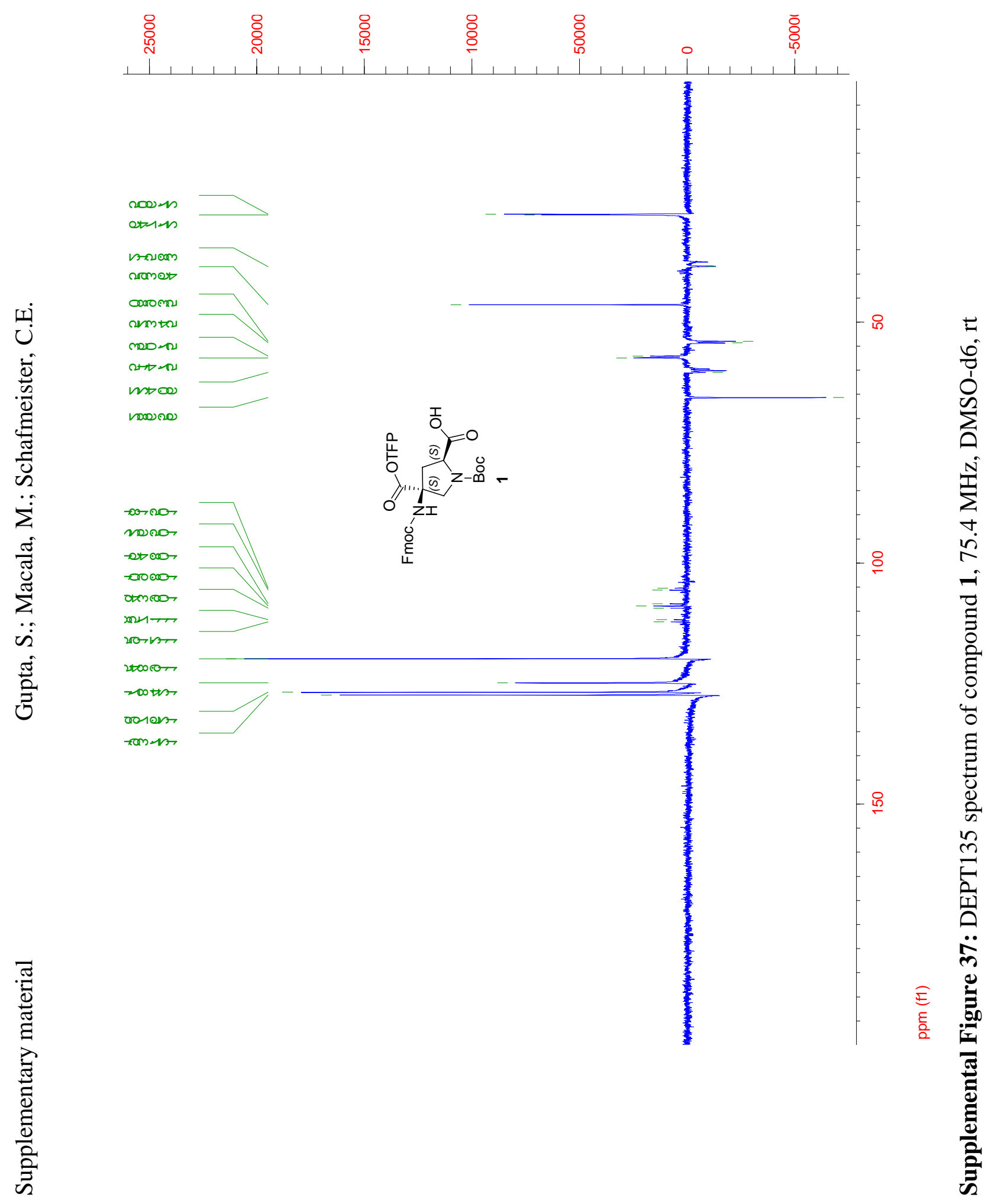




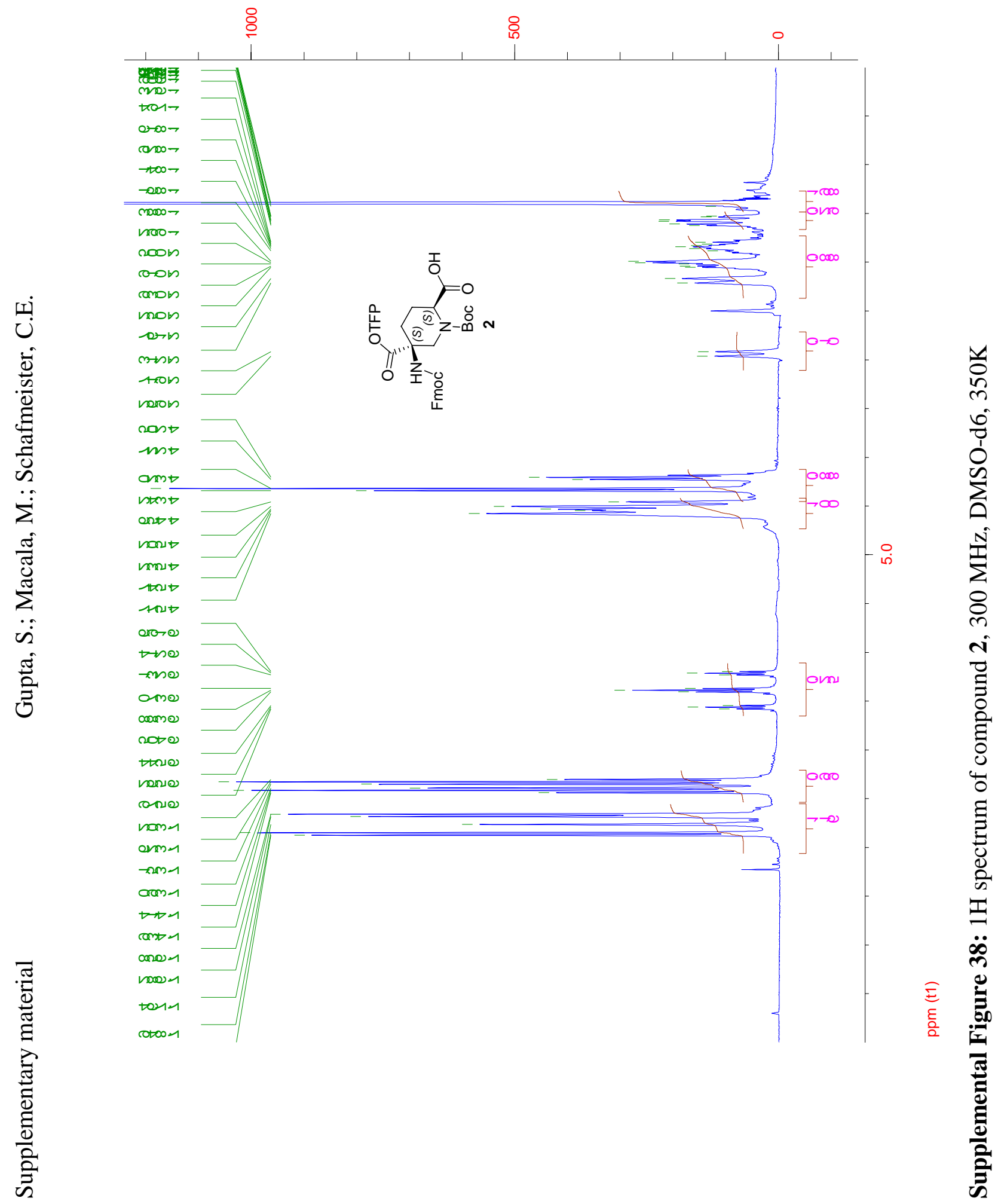




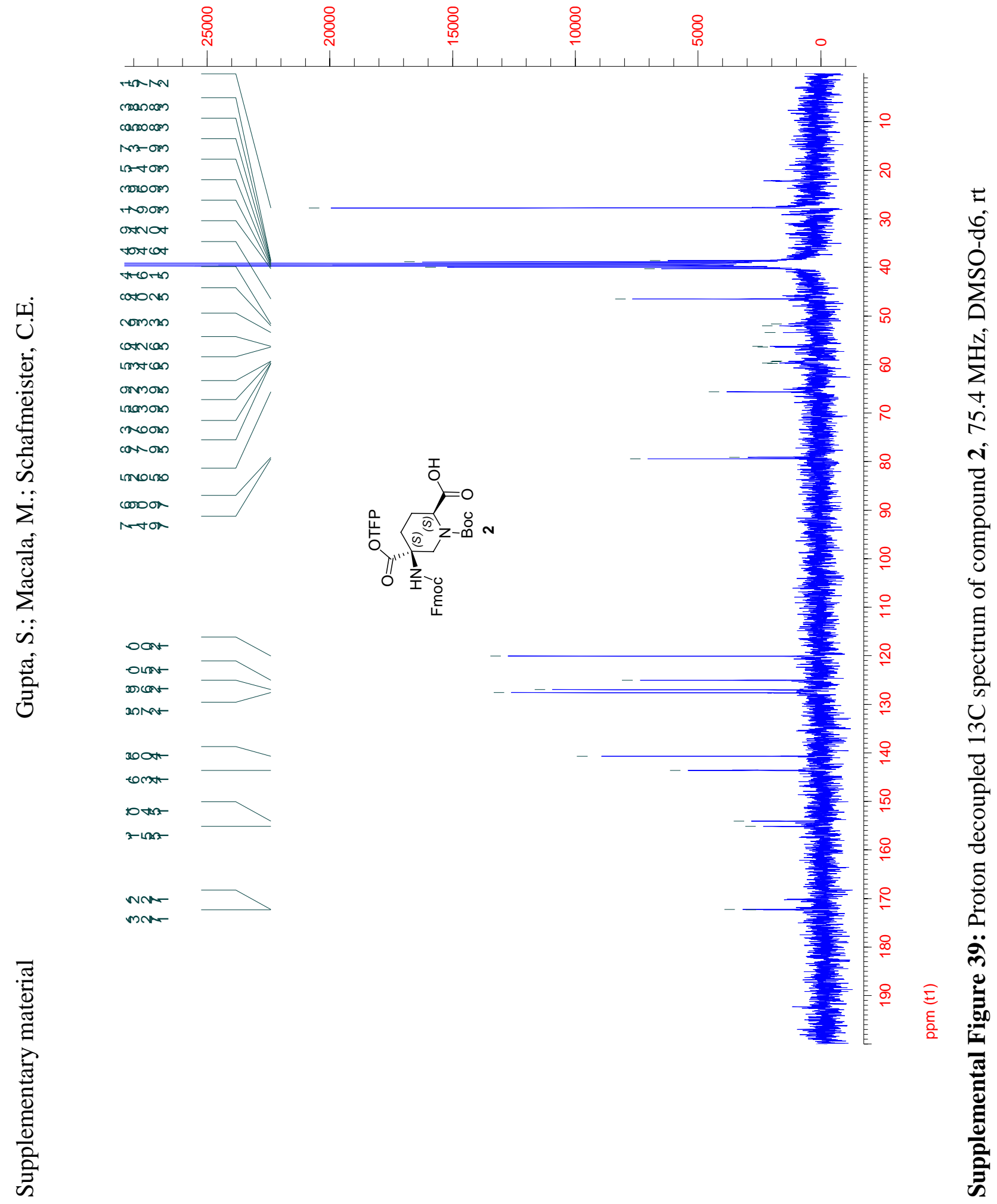




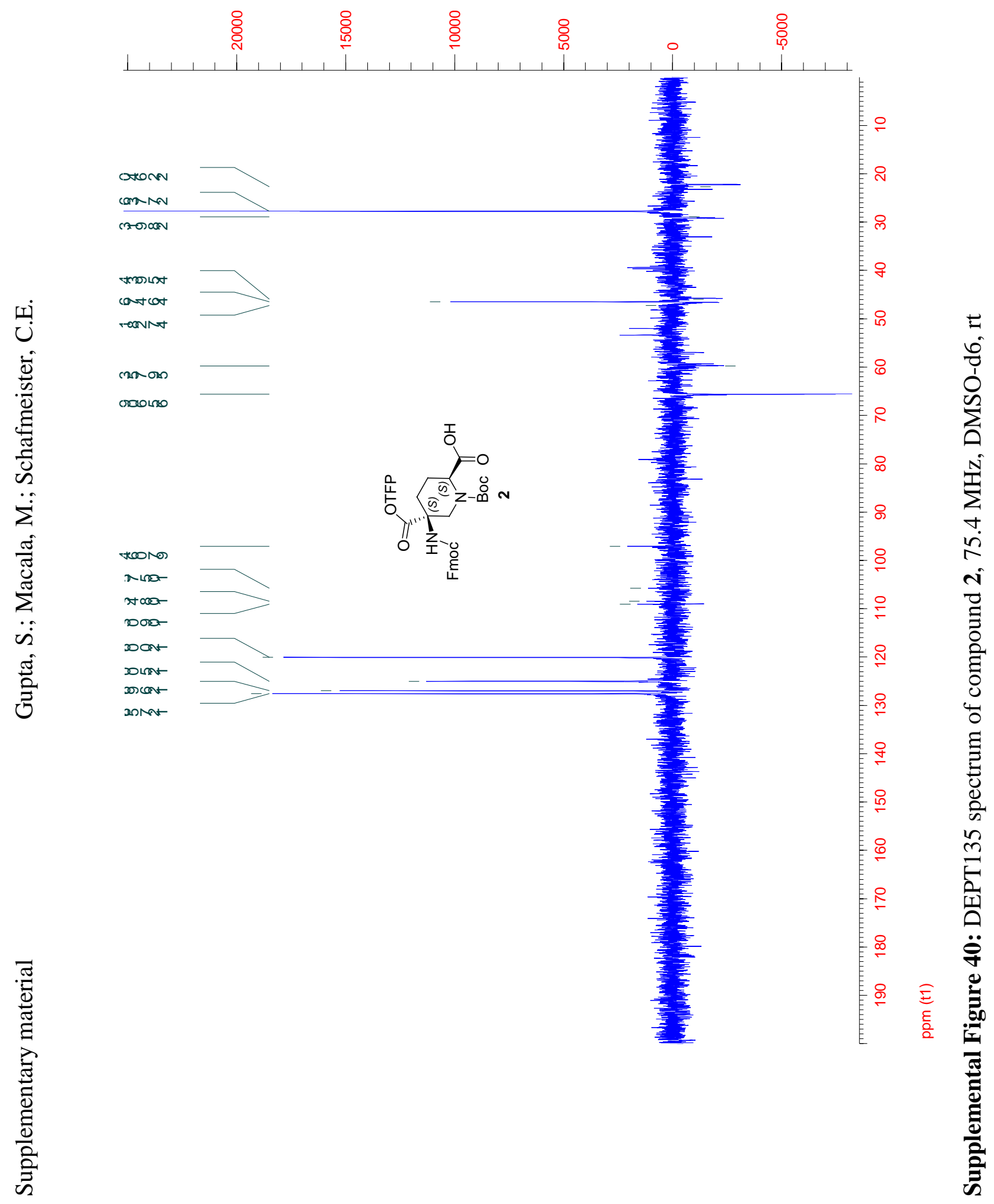




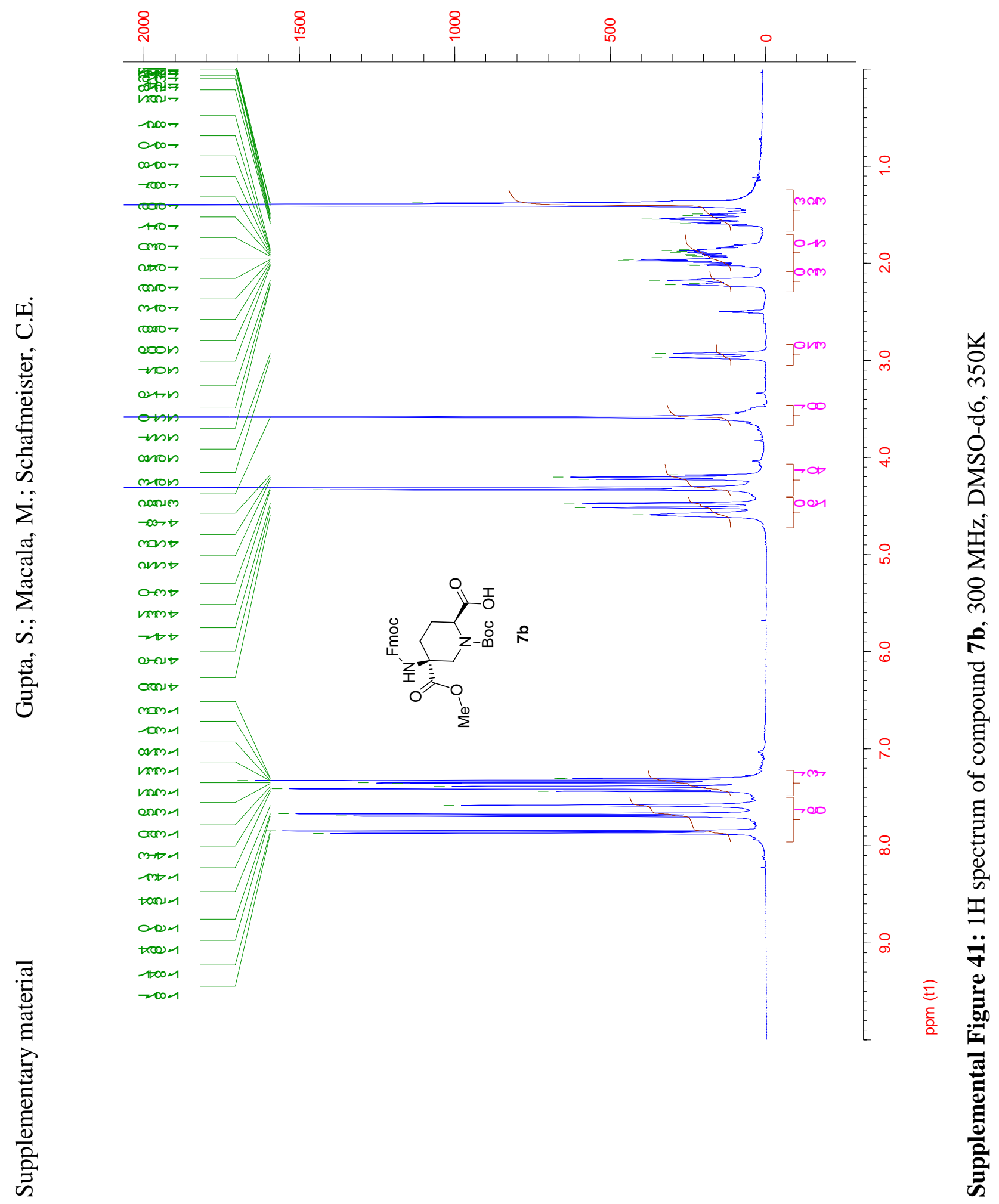




$$
1
$$




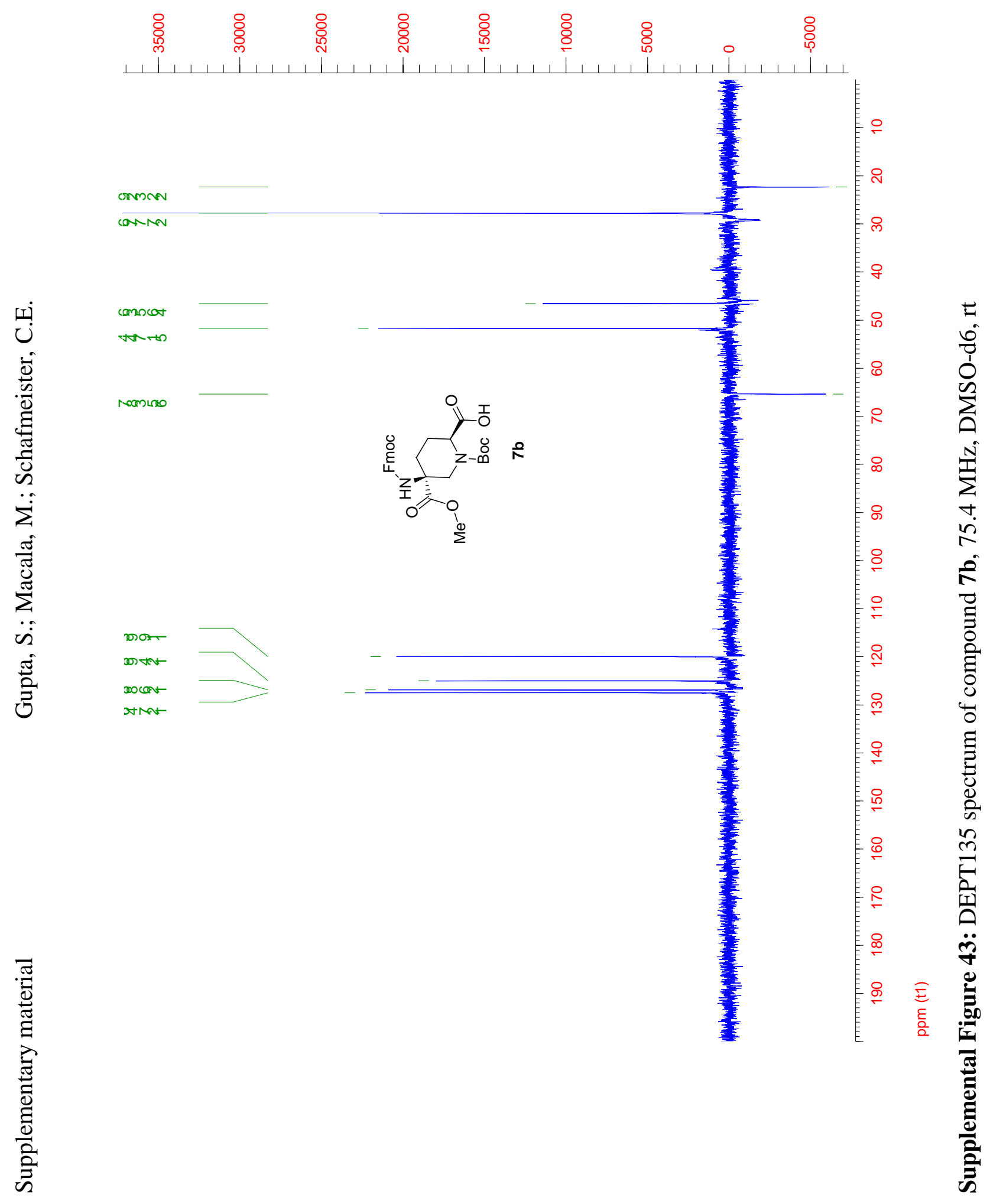

